University of Tennessee Health Science Center UTHSC Digital Commons

8-2019

\title{
Antibiotic Drug Discovery Targeting Bacterial Metabolism
}

Miranda J. Wallace

University of Tennessee Health Science Center

Follow this and additional works at: https://dc.uthsc.edu/dissertations

Part of the Medicine and Health Sciences Commons, Microbiology Commons, and the Molecular Biology Commons

\section{Recommended Citation}

Wallace, Miranda J. (0000-0003-3036-4545), "Antibiotic Drug Discovery Targeting Bacterial Metabolism" (2019). Theses and Dissertations (ETD). Paper 495. http://dx.doi.org/10.21007/etd.cghs.2019.0488.

This Dissertation is brought to you for free and open access by the College of Graduate Health Sciences at UTHSC Digital Commons. It has been accepted for inclusion in Theses and Dissertations (ETD) by an authorized administrator of UTHSC Digital Commons. For more information, please contact jwelch30@uthsc.edu. 


\title{
Antibiotic Drug Discovery Targeting Bacterial Metabolism
}

\begin{abstract}
Over the last century, the use of antibiotics has enabled many advances in modern medicine, making life as we know it possible. In recent years, however, emerging bacterial resistance to virtually all major antibiotic classes has resulted in a worldwide increase in morbidity, mortality, and financial burden associated with drug resistant infections. The antimicrobial resistance crisis presents an urgent need for new antimicrobials with distinct mechanisms of action from existing drugs. The current pharmaceutical pipeline of new antibiotics is limited due to three obstacles: a lack of understanding of resistance mechanisms, a dearth of novel mechanisms of action among new antibiotics, and drug discovery challenges unique to bacteria due to their cellular and physiological composition. My dissertation research addressed each of these challenges. The mechanisms of resistance to folate biosynthesis inhibitors in Staphylococcus aureus were explored from a genetic, biological, biochemical, and structural basis. Unexpected roles in resistance and fitness were attributed to various mutations in the sulfonamide target dihydropteroate synthase. This information currently guides the development of next-generation antifolates designed to avoid these characterized resistance strategies. In the subsequent chapter, a conditional metabolic screening approach was employed to discover inhibitors disrupting metabolic pathways related to folate biosynthesis. The testing conditions were optimized to measure the biological activity of antimetabolites, which is often masked by nutrients present in standard testing media. This screen yielded an inhibitor of cysteine synthase A in Escherichia coli, which was characterized in chapter four. Multiple experimental approaches yielded indications that the cysteine synthase $A$ inhibitors have a false-product mechanism, resulting in a widespread impact on several key branches of metabolism beyond cysteine biosynthesis. The final research chapter describes the adaptation of the cellular thermal shift assay to explore target engagement in the Gram-negative cell system. Drug entry and accumulation are especially challenging to achieve in Gram-negative cells due to their unique dual membrane system and associated efflux machinery. This assay provided an early stage tool to quickly assess the ability of antimicrobial candidates to engage the intended target in the intact cell system and also measure efflux sensitivity. Taken together, this dissertation contributes to the fight against the antimicrobial resistance crisis from multiple angles, all within the context of bacterial metabolism which is a rich source of exciting new antibiotic targets.
\end{abstract}

\section{Document Type}

Dissertation

Degree Name

Doctor of Philosophy (PhD)

Program

Biomedical Sciences

Research Advisor

Richard E. Lee, Ph.D.

\section{Keywords}

Antibiotic, Drug discovery, Drug resistance, Gram-negative bacteria, Metabolism, Staphylococcus aureus

\section{Subject Categories}

Medicine and Health Sciences | Microbiology | Molecular Biology 


\title{
Antibiotic Drug Discovery Targeting Bacterial Metabolism
}

\author{
A Dissertation \\ Presented for \\ The Graduate Studies Council \\ The University of Tennessee \\ Health Science Center
}

\author{
In Partial Fulfillment \\ Of the Requirements for the Degree \\ Doctor of Philosophy \\ From The University of Tennessee
}

By

Miranda J. Wallace

August 2019 
Copyright (C) 2019 by Miranda J. Wallace. All rights reserved. 


\section{ACKNOWLEDGEMENTS}

I would like to express my gratitude towards several special people in my life who have gone above and beyond in supporting me throughout my time as a doctoral student. Firstly, I would like to thank my doctoral advisor, Dr. Richard E. Lee, for allowing the opportunity to study under his guidance. I consider myself very fortunate to have had the special opportunity to work in the incredibly supportive environment Richard has cultivated that fosters the development of independent, creative, and clinically relevant research skills. I would like to also acknowledge all of my current and former lab mates in the Lee Lab. Among this community I have had an endless supply of intellectual, professional, and emotional support. I am also thankful to my dissertation committee. Each committee member has consistently provided unique and invaluable input and support that has vastly improved both my critical thinking skills and the outcomes of my dissertation research. Outside of the lab, the constant support of my family and my close friends from Arkansas have been a key component of my wellbeing and success throughout graduate school. Last but nowhere near least, I would like to express my sincere gratitude towards my husband Nick. His love, friendship, support, and cooking skills have carried me through the roller coaster of graduate school. I owe my success as a graduate student to this incredible community and their collective support has made this a memorable and intellectually enriching experience. 


\begin{abstract}
Over the last century, the use of antibiotics has enabled many advances in modern medicine, making life as we know it possible. In recent years, however, emerging bacterial resistance to virtually all major antibiotic classes has resulted in a worldwide increase in morbidity, mortality, and financial burden associated with drug resistant infections. The antimicrobial resistance crisis presents an urgent need for new antimicrobials with distinct mechanisms of action from existing drugs. The current pharmaceutical pipeline of new antibiotics is limited due to three obstacles: a lack of understanding of resistance mechanisms, a dearth of novel mechanisms of action among new antibiotics, and drug discovery challenges unique to bacteria due to their cellular and physiological composition. My dissertation research addressed each of these challenges. The mechanisms of resistance to folate biosynthesis inhibitors in Staphylococcus aureus were explored from a genetic, biological, biochemical, and structural basis. Unexpected roles in resistance and fitness were attributed to various mutations in the sulfonamide target dihydropteroate synthase. This information currently guides the development of next-generation antifolates designed to avoid these characterized resistance strategies. In the subsequent chapter, a conditional metabolic screening approach was employed to discover inhibitors disrupting metabolic pathways related to folate biosynthesis. The testing conditions were optimized to measure the biological activity of antimetabolites, which is often masked by nutrients present in standard testing media. This screen yielded an inhibitor of cysteine synthase A in Escherichia coli, which was characterized in chapter four. Multiple experimental approaches yielded indications that the cysteine synthase A inhibitors have a false-product mechanism, resulting in a widespread impact on several key branches of metabolism beyond cysteine biosynthesis. The final research chapter describes the adaptation of the cellular thermal shift assay to explore target engagement in the Gram-negative cell system. Drug entry and accumulation are especially challenging to achieve in Gram-negative cells due to their unique dual membrane system and associated efflux machinery. This assay provided an early stage tool to quickly assess the ability of antimicrobial candidates to engage the intended target in the intact cell system and also measure efflux sensitivity. Taken together, this dissertation contributes to the fight against the antimicrobial resistance crisis from multiple angles, all within the context of bacterial metabolism which is a rich source of exciting new antibiotic targets.
\end{abstract}




\section{TABLE OF CONTENTS}

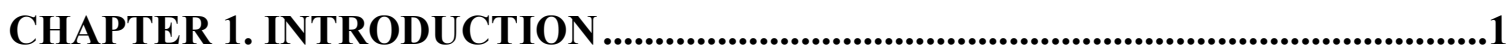

Discovery and Establishment of Antimicrobials in Modern Medicine ...........................

The Rise of Antibiotic Resistance ........................................................................... 3

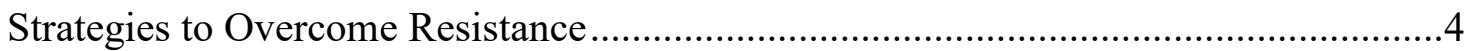

Folate Biosynthesis Is an Effective Target for Existing and Experimental

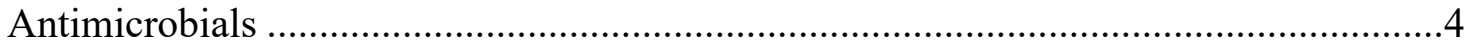

Antibiotic Discovery Towards Cysteine Biosynthesis ................................................ 7

Drug Discovery Challenges Unique to GN Bacteria ...............................................

Improving Antibiotic Drug Discovery by Engaging Critical Metabolic Pathways .........9

\section{CHAPTER 2. ANALYSIS OF CHROMOSOMAL RESISTANCE} MUTATIONS IN STAPHYLOCOCCUS AUREUS ..........................................11

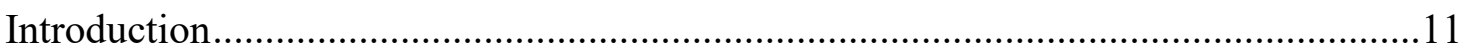

Sulfonamide resistance in $S$. aureus and other clinically relevant species ................11

Improved understanding of DHPS structure and function in recent decades ............12

Recent insights into the FBP function and candidacy for next-generation

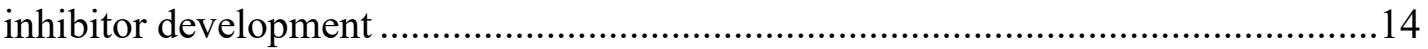

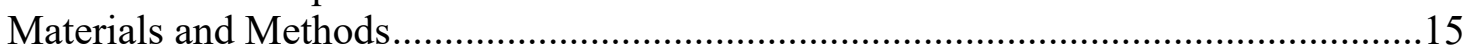

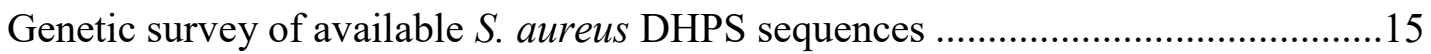

Heterologous expression and purification of recombinant DHPS variants ..............15

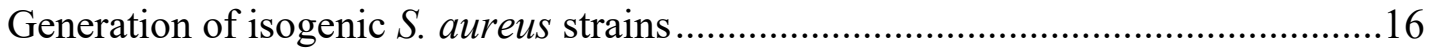

Minimum inhibitory concentration (MIC) determination....................................... 16

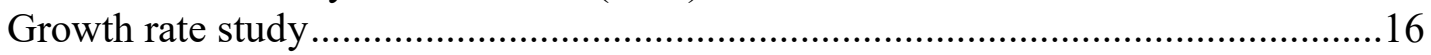

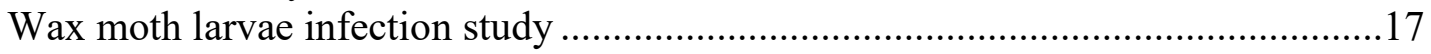

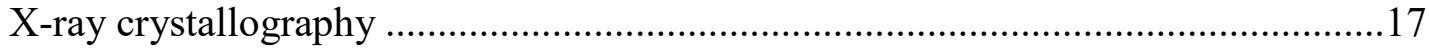

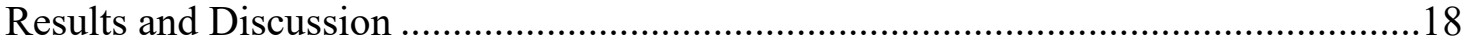

Identification of five sulfonamide resistance mutations in S. aureus DHPS .............18

Identification of homologous resistance mutations in other species ........................18

In vitro characterization of DHPS enzymes harboring sulfonamide resistance

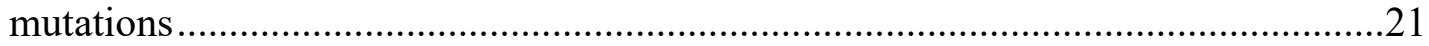

Resistance profile of an $S$. aureus isogenic DHPS mutant panel against

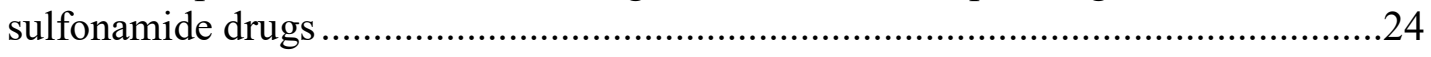

Altered susceptibility of isogenic mutants to TMP and co-trimoxazole...................26

Growth curve studies ...............................................................................28

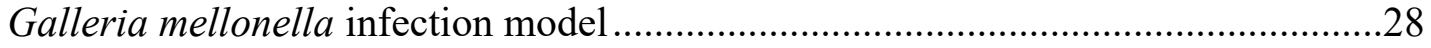

Structural characterization of the DHPS resistance mutations ...............................31

Conclusions and Future Perspectives .................................................................33

CHAPTER 3. METABOLIC PROFILING OF ANTIFOLATES LEADS TO A SCREEN FOR NEW INHIBITORS OF BACTERIAL METABOLISM...................35

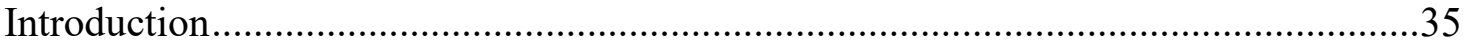

Metabolic networks associated with tetrahydrofolate are promising avenues for

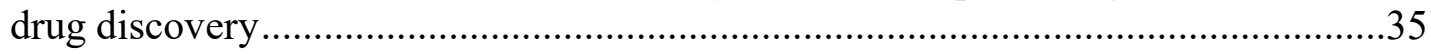




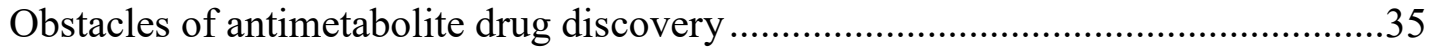

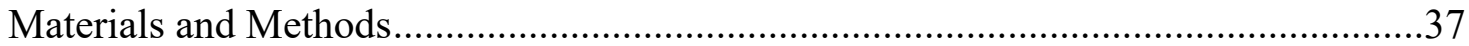

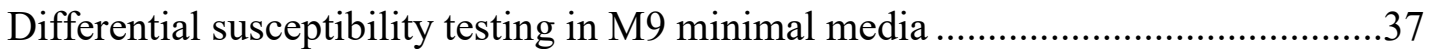

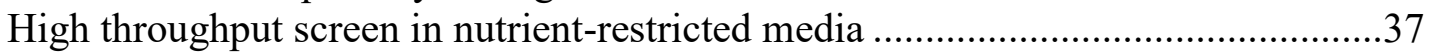

Secondary validation of primary hits and testing for rescue effect..........................38

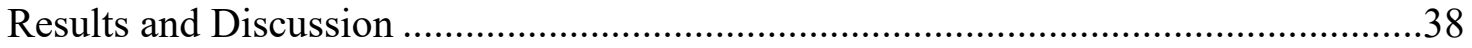

Minimal media conditions enable SAR study of pterin-sulfa conjugates..................38

Assay validation for the conditional metabolic screen through a preliminary screen of known Bioactive compounds .............................................................4 41

High-throughput screen of nearly 13,000 compounds ........................................43

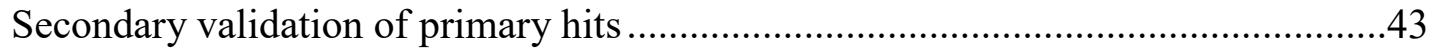

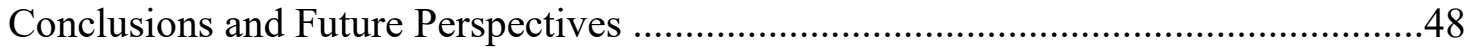

\section{CHAPTER 4. DISCOVERY AND DEVELOPMENT OF THIOACETAMIDE- LINKED 1,2,3-TRIAZOLES TARGETING CYSTEINE SYNTHASE A................50}

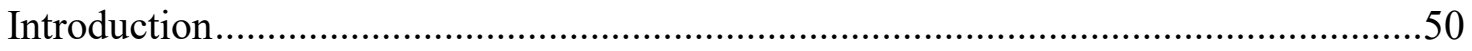

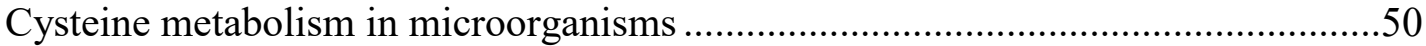

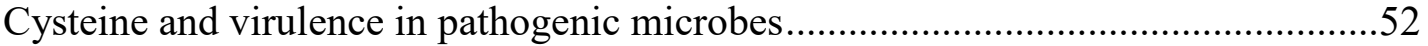

Current antibiotic drug discovery efforts towards CysK ....................................52

Materials and Methods......................................................................................5

Spontaneous mutant generation and genomic sequencing ..................................53

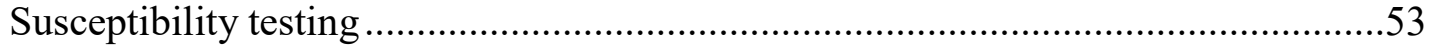

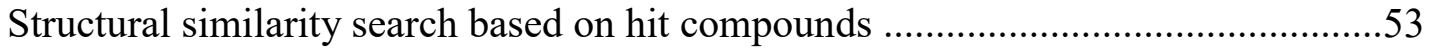

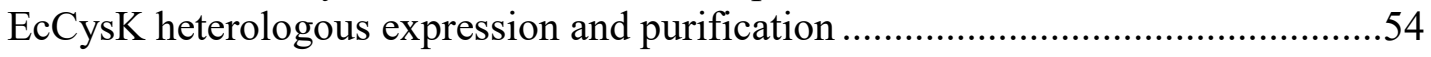

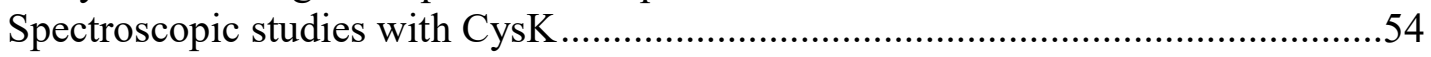

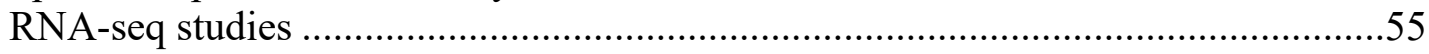

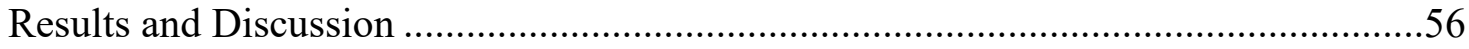

Initial characterization of the TAT hits and target determination............................56

Modeling of observed resistance mutations on the E. coli CysK enzyme.................56

Homology of resistance mutations in the CysK enzyme of various species ............60

Cellular studies further confirm CysK as the target of lead inhibitors ....................60

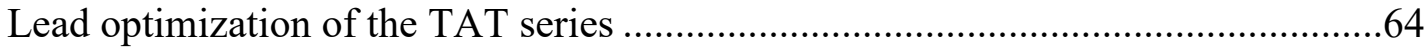

Antagonism of MIC by metabolites closely related to L-cysteine biosynthesis........67

TATs potentiate DHPS inhibitors ..................................................................67

TATs maintain activity against antifolate-resistant GN clinical isolates..................70

In vitro interactions of the TATs with the CysK enzyme .................................... 70

The transcriptional response of 3866-treated E. coli cells ......................................73

Conclusions and Future Perspectives ................................................................ 79

\section{CHAPTER 5. EXPLORING THE INTRACELLULAR TARGET}

ENGAGEMENT OF ANTIFOLATES IN GRAM-NEGATIVE BACTERIA ..........81

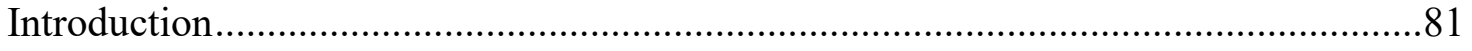

Molecular methods to explore target engagement in Gram-negative cells................81

Next-generation inhibitor development of antifolates ..........................................8.

Major efflux systems in Gram-negative bacteria .................................................82

Improved strategies to develop GN-effective antifolates ....................................83 


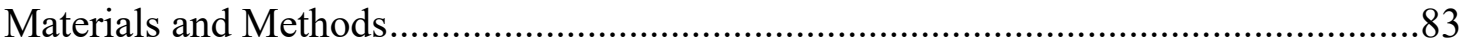

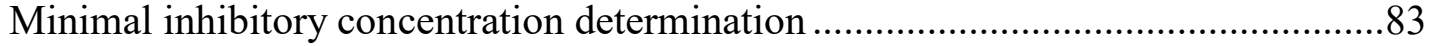

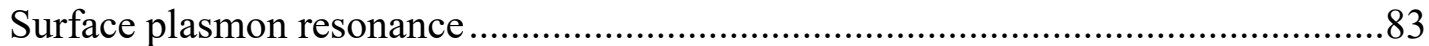

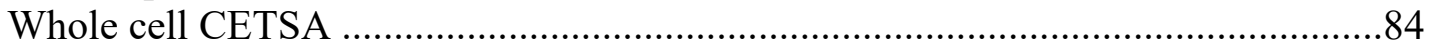

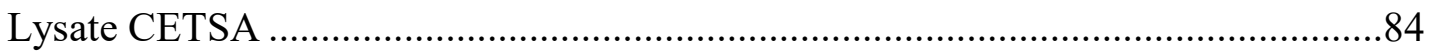

Lysate ITDRF

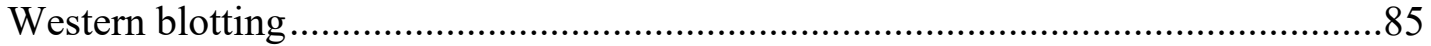

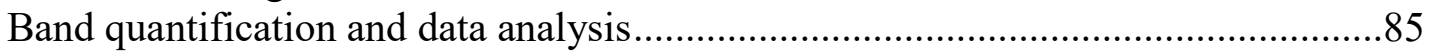

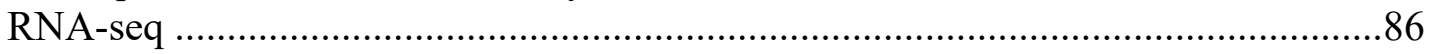

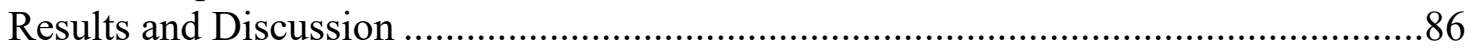

Selection of molecules to test in the GN CETSA model ..........................................86

Binding kinetics analysis of DHPS compounds using surface plasmon resonance...88

Generation of $E$. coli strains constitutively expressing DHFR and DHPS .................88

Susceptibility profile of the E. coli CETSA panel against established and

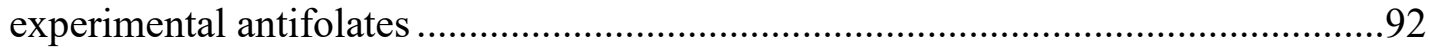

Optimization of western blotting conditions for CETSA panel.................................92

Establishment of CETSA melting curves for DHFR and DHPS ...............................94

DHPS lysate CETSA reveals antifolate affinity profile..............................................94

Addressing permeability issues with pyridazine inhibitors of DHPS .........................99

Global transcriptomic effects of antifolates ...........................................................99

Assessing susceptibility to efflux with novel DHFR inhibitors................................102

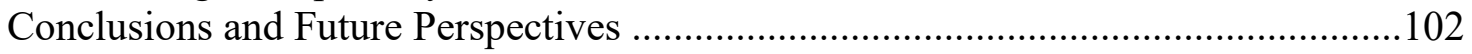

\section{CHAPTER 6. CONTRIBUTIONS TO THE FIELD AND LOOKING}

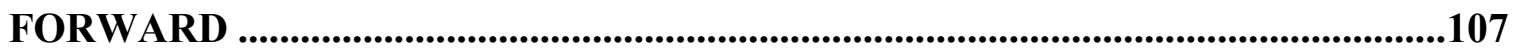

LIST OF REFERENCES .....................................................................................................109

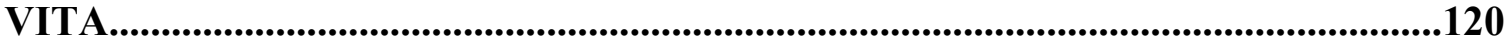




\section{LIST OF TABLES}

Table 2-1. Categorization of DHPS amino acid variations identified in this study.......19

Table 2-2. Survey of DHPS sequence variations and reported sulfonamide susceptibility.

Table 2-3. Occurrence of identified sulfonamide resistance mutations in other pathogenic species

Table 2-4. Biochemical profile of SaDHPS variant enzymes. 23

Table 2-5. $\quad \mathrm{MIC}_{80}$ Values for $S$. aureus Isogenic DHPS Mutant Panel $(\mu \mathrm{g} / \mathrm{mL})$. 25

Table 3-1. The MICs of pterin-sulfa conjugates can be rescued by metabolites related to folate biosynthesis.

Table 3-2. Validated hits from the conditional metabolic screen................................46

Table 4-1. Preliminary MICs and resistance frequency of the TAT hits. .....................57

Table 4-2. MIC shifts of spontaneous mutants at 24 hours.....................................58

Table 4-3. Chromosomal mutations in spontaneous E. coli K12 mutants raised against the TATs.

Table 4-4. Strains used for characterization of the TATs. ...........................................63

Table 4-5. PK/PD Characterization of Lead TATs. ....................................................66

Table 4-6. Susceptibility testing of TATs in the presence of metabolites related to cysteine biosynthesis. .68

Table 4-7. Susceptibility of $E$. coli $\Delta$ CysK to various antibiotic compounds. .68

Table 4-8. Lead CysK inhibitors are effective against antifolate-resistant GN clinical isolates.

Table 5-1. $\quad B a$ DHPS SPR data for DHPS-targeted compounds..................................89

Table 5-2. $\quad$ Strains used in the GN CETSA study................................................. 91

Table 5-3. $\quad \mathrm{MIC}_{80 \mathrm{~S}}$ of $E$. coli CETSA panel in M9 minimal media $(\mu \mathrm{M})$....................93

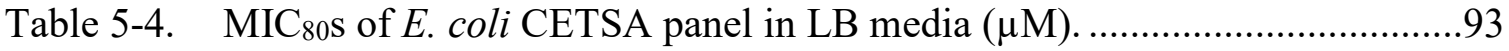




\section{LIST OF FIGURES}

Figure 1-1 Timeline of deployment of major antibiotic classes and the general cell

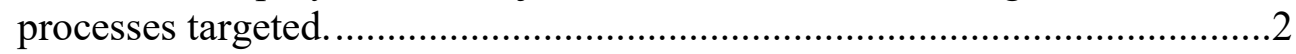

Figure 1-2. The folate biosynthesis pathway...........................................................6

Figure 1-3. Cysteine biosynthesis and associated pathways in prokaryotes and mammals.

Figure 1-4. The Gram-negative cell membrane system..................................................10

Figure 2-1. Structure and catalytic mechanism of DHPS..............................................13

Figure 2-2. Sequence comparison of DHPS from S. aureus and other key pathogenic species.

Figure 2-3. Trimethoprim susceptibility profile of isogenic DHPS mutant panel. .........27

Figure 2-4. Doubling times of the isogenic DHPS mutant panel in S. aureus................29

Figure 2-5. Optimization of wax moth larvae infection model. ......................................29

Figure 2-6. Galleria mellonella virulence and rescue study...........................................30

Figure 2-7. Structural characterization of the WT and F17L E208K salt bridge array...32

Figure 3-1. Z factors observed for the preliminary Bioactives screen.

Figure 3-2. Growth inhibition and antagonism by key metabolites observed by known antimicrobials in the Bioactives collection.

Figure 3-3. HTS of nearly 13,000 compounds in nutrient-restricted broth......................44

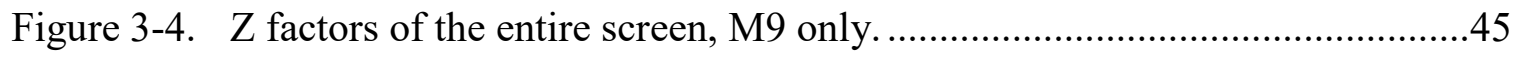

Figure 3-5. Thymidylate synthase is closely linked to the FBP .....................................47

Figure 4-1. Cysteine metabolism and related pathways in microorganisms. ..................51

Figure 4-2. Thioacetamide-linked 1,2,3-triazole hits from the metabolic screen............57

Figure 4-3. Location of spontaneous mutations on E. coli CysK...................................61

Figure 4-4. Sequence alignment of CysK and location of mutations induced spontaneously for resistance to TATs.

Figure 4-5. Cell-based studies to investigate the putative CysK target. 
Figure 4-6. TAT molecules representing the SAR study.

Figure 4-7. 3900 potentiates inhibitors of DHPS.

Figure 4-8. Spectroscopic profiling of EcCysK in combination with native substrate and CysK inhibitors......

Figure 4-9. Dose-dependent spectroscopic effects.

Figure 4-10. Volcano plot of global transcriptional changes in M9-based media with taurine as a sulfur source compared to magnesium sulfate as the sulfur source.

Figure 4-11. Volcano plots of transcriptional responses to TAT treatment. .77

Figure 4-12. E. coli pathway networks transcriptionally altered by treatment with 3866.

Figure 5-1. Established and experimental antifolates selected for the CETSA study.....87

Figure 5-2. SPR sensograms for the DHPS-targeted molecules.

Figure 5-3. Western blot of lysates prepared from full CETSA panel and background strains...

Figure 5-4. Preliminary whole cell CETSA covering a broad temperature range...........96

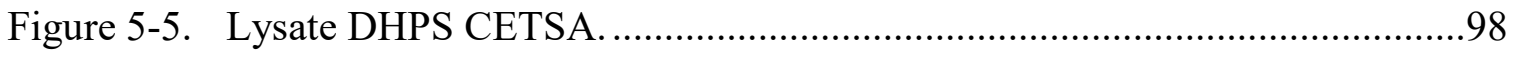

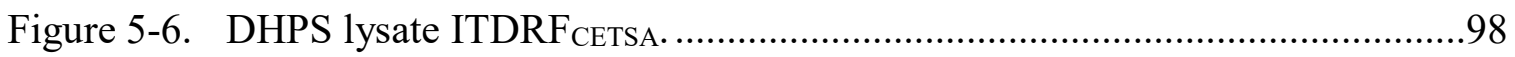

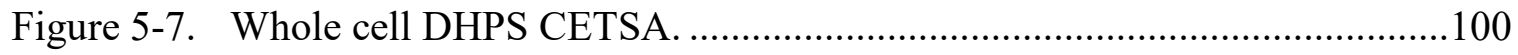

Figure 5-8. Volcano plots of RNA-seq study of E. coli treated with 568 and 3893.....101

Figure 5-9. Venn diagrams of shared genes transcriptionally altered between DHPS inhibitors and the CysK inhibitor 3866.

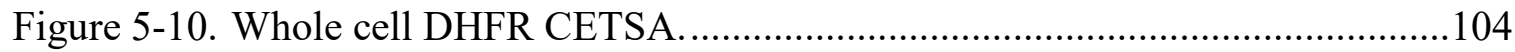

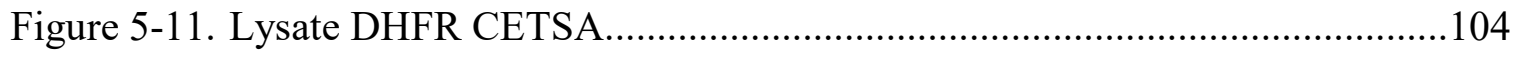




\section{LIST OF ABBREVIATIONS}

\begin{tabular}{|c|c|}
\hline AMR & Antimicrobial Resistance \\
\hline CAM & Chloramphenicol \\
\hline CETSA & Cellular Thermal Shift Assay \\
\hline CysK & Cysteine Synthase A \\
\hline CysM & Cysteine Synthase B \\
\hline DHFR & Dihydrofolate Reductase \\
\hline DHPP & Dihydropterin Pyrophosphate \\
\hline DHPS & Dihydropteroate Synthase \\
\hline DMSO & Dimethyl Sulfoxide \\
\hline EC50 & Half Maximal Effective Concentration \\
\hline FBP & Folate Biosynthesis Pathway \\
\hline GN & Gram-negative \\
\hline GP & Gram-positive \\
\hline HTS & High-Throughput Screening \\
\hline ITDRF & Isothermal Dose Response Fingerprint \\
\hline LD50 & Median Lethal Dose \\
\hline MDR & Multidrug Resistance \\
\hline $\mathrm{MIC}$ & Minimal Inhibitory Concentration \\
\hline MOA & Mechanism of Action \\
\hline MRSA & Methicillin Resistant Staphylococcus aureus \\
\hline OALS & $O$-acetyl-L-serine \\
\hline OD & Optical Density \\
\hline$p \mathrm{ABA}$ & Para-aminobenzoic Acid \\
\hline $\mathrm{PK} / \mathrm{PD}$ & Pharmacokinetic/Pharmacodynamic \\
\hline PPi & Pyrophosphate \\
\hline RSAP & Reductive Sulfate Assimilation Pathway \\
\hline SAR & Structure Activity Relationship \\
\hline SAT & Serine Acetyltransferase \\
\hline SMX & Sulfamethoxazole \\
\hline SPR & Surface Plasmon Resonance \\
\hline THF & Tetrahydrofolate \\
\hline $\mathrm{T}_{\mathrm{AGG}}$ & Aggregation Temperature \\
\hline $\mathrm{T}_{\mathrm{M}}$ & Melting Temperature \\
\hline TMP & Trimethoprim \\
\hline
\end{tabular}




\section{CHAPTER 1. INTRODUCTION}

\section{Discovery and Establishment of Antimicrobials in Modern Medicine}

Penicillin is often regarded as the first antibiotic discovered in the modern era. In 1928 an agar plate in the laboratory of Alexander Fleming was serendipitously contaminated with a Penicillium mold species that inhibited the growth of the pathogenic Staphylococci originally inoculated on the plate (Fleming 1929). Penicillin was eventually isolated and identified as the growth inhibitory component produced by the mold by Ernst Chain and Howard Florey (Chain 1940). In the early 1940s, penicillin proved to be an effective treatment for human infections and does in fact represent the earliest antibiotic derived from a natural product (Abraham 1941).

Before the discovery of penicillin, however, two very important antimicrobial chemotherapies had already been identified and deployed for treatment of human infections. The earliest of these discoveries was arsphenamine, which represents the first effective syphilis treatment (Williams 2009). Arsphenamine was originally synthesized by Alfred Bertheim and found to be effective against spirochetes by Paul Ehrlich and his assistants (Strebhardt 2008). This compound showed efficacy in clearing human syphilis infections as early as 1909 and would become the standard of care for syphilis treatment until penicillin was widely available in the 1940s (Williams 2009).

Another antimicrobial discovered before penicillin was prontosil, which was originally synthesized as a dye in 1909 at the University of Vienna. It wasn't until 1932 that this agent was found by Gerhard Domagk to clear Streptococcus pyogenes infections in mice. Shortly after this discovery, prontosil showed promise in treating human infections as early as 1935 (Chain 1940). It was later discovered that a colorless component of the prontosil molecule, sulfanilamide, was active on its own (Trefouel 1935). Prontosil is metabolized in vivo to produce the active sulfanilamide component (Mitscher 1995). Several sulfonamide derivatives have been generated since the discovery of sulfanilamide, and this antibiotic class proved invaluable for war wounds and systemic infections (Sköld 2000). Sulfonamides remain a cornerstone treatment option for a variety of infection types today.

These early discoveries of arsphenamine, prontosil and penicillin spurred the "Golden Age" of antibiotic drug discovery, which would last through the 1970s (Figure 1-1). Empirical screening efforts yielded numerous natural product-derived antibiotics including $\beta$-lactams, aminoglycosides macrolides, tetracyclines, ansamycins, and glycopeptides. Synthetic antibiotics were also generated, including nitrofurans, isoniazid, and trimethoprim (TMP) (Silver 2011). This antimicrobial armamentarium developed since the early 1900s has enabled the development of modern medicine as we know it and has vastly improved global human health. Antimicrobials allow for improved management of simple infections and communicable disease. Advanced medical procedures and treatment regimens including chemotherapy, surgeries, and dialysis, which render patients more vulnerable to infections, could be developed successfully 


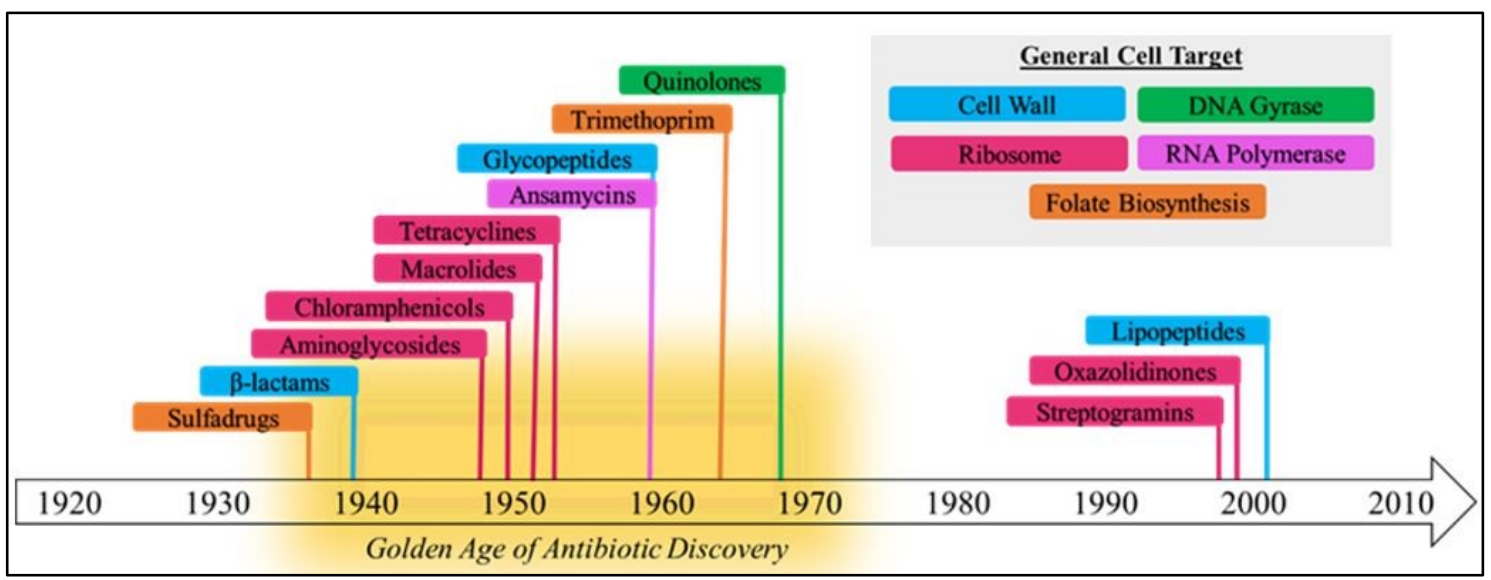

Figure 1-1 Timeline of deployment of major antibiotic classes and the general cell processes targeted.

Major antibiotic classes are color-coded according to their general cell target. 
with the help of antimicrobial therapies (Ventola 2015).

\section{The Rise of Antibiotic Resistance}

The deployment of new antibiotic classes to clinical use has slowed since the 1970s, although a few notable narrow spectrum agents have been introduced in recent years (Figure 1-1) (Silver 2011, Lewis 2017). This decline in antibiotic discovery has been concurrent with an increase in resistant infections in recent decades, resulting in a global antimicrobial resistance (AMR) crisis. AMR is presently one of the largest global health threats in the 21 st century and imposes a high societal burden in terms of morbidity, mortality, and financial loss. Approximately 2 million cases of AMR infections occur annually in the United States resulting in 23,000 AMR-associated deaths each year (Centers for Disease Control and Prevention 2013). It is estimated that without significant intervention, the global death rate attributed to AMR could reach 10 million deaths per year by 2050 and surpass death rates attributed to cancer (O'Neill 2016). An alarming phenomenon is the increasing resistance rates observed against "last-resort" antibiotic treatment options such as carbapenems and colistin among Gram-negative (GN) pathogens (Tacconelli 2018). The resistance increasingly observed against virtually every antibiotic class has introduced the possibility of entering a "post-antibiotic era," wherein antibiotic therapy is rendered ineffective and much of the progress made with modern medicine over the last century is reversed (Alanis 2005).

Several factors have contributed to the current AMR crisis. An increasingly "global" lifestyle wherein trade and travel are more commonplace has accommodated the spread of resistant microbes. Antibiotic overuse in agriculture and aquaculture has allowed for the introduction of antibiotics into the environment which encourages an environmental reservoir of resistance genes. Antibiotics are often overprescribed in the clinic and underregulated in developing countries, resulting in more frequent and unnecessary use in humans. This overuse of antibiotics encourages resistance in both community and hospital settings (Ventola 2015).

In response to antibiotic stress encountered in vivo or in the environment, microorganisms readily employ numerous resistance mechanisms including target modification, drug deactivation, efflux, and metabolic bypass of the antibiotic target. AMR can be attributed to both resistance mechanisms evolved in direct response to antibiotic exposure, or to inherent resistance mechanisms (Wright 2010). Resistance genes are oftentimes rapidly disseminated in microbial populations through both vertical transfer to daughter cells and horizontal transfer of plasmids. Subsets of microbial populations can assume a "persister" state, wherein they assume a dormant state and are highly tolerant to antibiotics within chronic infection types and biofilms (Lewis 2010). 


\section{Strategies to Overcome Resistance}

To effectively manage the global health crisis of AMR, improvements must be made towards the ability to understand, monitor, and treat AMR infections. Surveillance of AMR pathogens and resistance genes in the clinic, the community, and the environment is critical for the detection, containment, and prevention of resistant isolates (Tacconelli 2018). Emerging rapid diagnostics technologies yield significant promise towards fast and accurate detection of the pathogenic species to allow for more targeted and prudent antimicrobial treatment regimens. Reducing the use of antibiotics in agriculture and aquaculture as well as higher regulation on antibiotic production and use outside the clinic in developing countries would certainly reduce the development and dissemination of AMR (Wright 2010).

A remaining strategy to overcome AMR that is addressed in this dissertation is to develop desperately needed new and effective antibiotic therapies. The pharmaceutical pipeline for new antibiotics has presented a strikingly low output of new antimicrobial classes since the 1970s (Figure 1-1). Although three new antibiotic classes have been introduced in the past couple of decades (streptogramins, oxazolidinones, and lipopeptides), these agents are narrow spectrum (Lewis 2017). New and effective antimicrobials for GN species are desperately needed in light of the rapid emergence of resistance to antibiotics of "last resort." Campaigns to develop new antimicrobial agents, whether they are designed to target established or new antibiotic targets, need to be developed with avoidance of known resistance mechanisms in mind.

Another issue with the existing antibiotic arsenal and even the ongoing pharmaceutical pipeline is that these agents generally target a discrete set of general cell processes including the bacterial cell wall, protein biosynthesis, and DNA biosynthesis (Figure 1-1). Bacterial metabolism remains an underexploited avenue for antibiotic development that has yet to be fully explored (Zlitni 2013). Although microorganisms and humans often share the same metabolic requirements for basic cell functions, microbes are evolutionarily distinct from mammals and often have unique biosynthetic routes to obtain necessary metabolites. Several enzymes within these pathways are structurally distinct or entirely absent in human systems, presenting them as ideal candidates for drug development with a low likelihood of interaction with homologous host targets. Antifolates are an existing antibiotic class which takes advantage of differences in folate metabolism between microbes and humans (Bermingham 2002). Other metabolic pathways are being investigated for similar antimetabolite mechanisms of action (Zlitni 2013).

\section{Folate Biosynthesis Is an Effective Target for Existing and Experimental Antimicrobials}

The folate biosynthetic pathway (FBP) plays a central role in the biosynthesis of several metabolites necessary for basic cell function and survival. The final product of this pathway is tetrahydrofolate (THF), which can act as a one-carbon donor during the 
biosynthesis of purines, thymine, and methionine. Furthermore, THF can act as a onecarbon acceptor in various degradation pathways (Bermingham 2002). THF and its derivatives play key roles in both the one-carbon cycle and the folate cycle.

The first committed step of the pathway is the conversion of GTP to a pterin ring which is catalyzed by GTP cyclohydrolase (Bermingham 2002) (Figure 1-2). This is followed by three enzymes catalyzing kinase, aldolase, and pyrophosphokinase reactions to form 6-hydroxymethyl-7,8-dihydropterin pyrophosphate (DHPP). Dihydropteroate synthase (DHPS) forms 7,8-dihydropteroate from DHPP and para-aminobenzoic acid (pABA), which is a product of the chorismate pathway (Dosselaere 2001). Glutamate is added to the dihydropteroate, forming dihydrofolate. The final enzyme, dihydrofolate reductase (DHFR), converts dihydrofolate to THF (Figure 1-2). The first four enzymes in the pathway, ending with DHPS, are absent in mammals. Mammals obtain folates from their diet, and therefore lack the upstream enzymes of this pathway. Many microorganisms rely on the de novo biosynthesis of THF and lack the associated folate uptake machinery.

Antifolates have held immense utility as broad-spectrum antibiotics for over 80 years (Domagk 1935). Antifolate antimicrobials target the upstream enzyme DHPS, which is absent in humans, as well as DHFR. DHFR is present in both humans and prokaryotes, however the antibiotic TMP has a much greater affinity for the bacterial enzyme (Brogden 1982). A commonly prescribed antimicrobial antifolate therapy is cotrimoxazole, which is a combination of sulfamethoxazole (SMX) and TMP which targets DHPS and DHFR, respectively. The two drugs show high synergy in combination and are effective against a broad spectrum of microbial species. Co-trimoxazole is commonly prescribed for urinary tract infections, ear infections, bronchitis, and pneumonia caused by Pneumocystis jirovecii (Bermingham 2002).

There are various other antifolate antibiotics in addition to the co-trimoxazole combination. Several sulfonamide derivatives have been generated since the discovery of sulfanilamide with improved efficacy and reduced host toxicity (Mitscher 1995). A key treatment option for multidrug resistant tuberculosis is para-amino salicylic acid (PAS) which acts as a prodrug activated by DHPS and ultimately inhibiting DHFR (Zheng 2013). Dapsone is another DHPS inhibitor that acts as a second-line treatment for Pneumocystis pneumonia and leprosy (Hughes 1998).

As with other antibiotic classes, resistance has compromised the utility of antifolates, causing many of these agents to be relegated to second-line treatment options. Resistance to sulfonamides and TMP is primarily achieved through target modification among Gram-positive (GP) and Plasmodium species (Sköld 2000). GN bacteria are notorious for gaining antifolate resistance through resistance plasmids encoding a resistant variant of either DHPS or DHFR, or through overexpression of the DHFR enzyme (Palmer 2014). Despite these observed resistance mechanisms, the immense clinical and historical utility of the FBP as an antimicrobial target has inspired several ongoing campaigns to develop next-generation antifolate inhibitors with the goal of avoiding known resistance mechanisms (Bermingham 2002). 


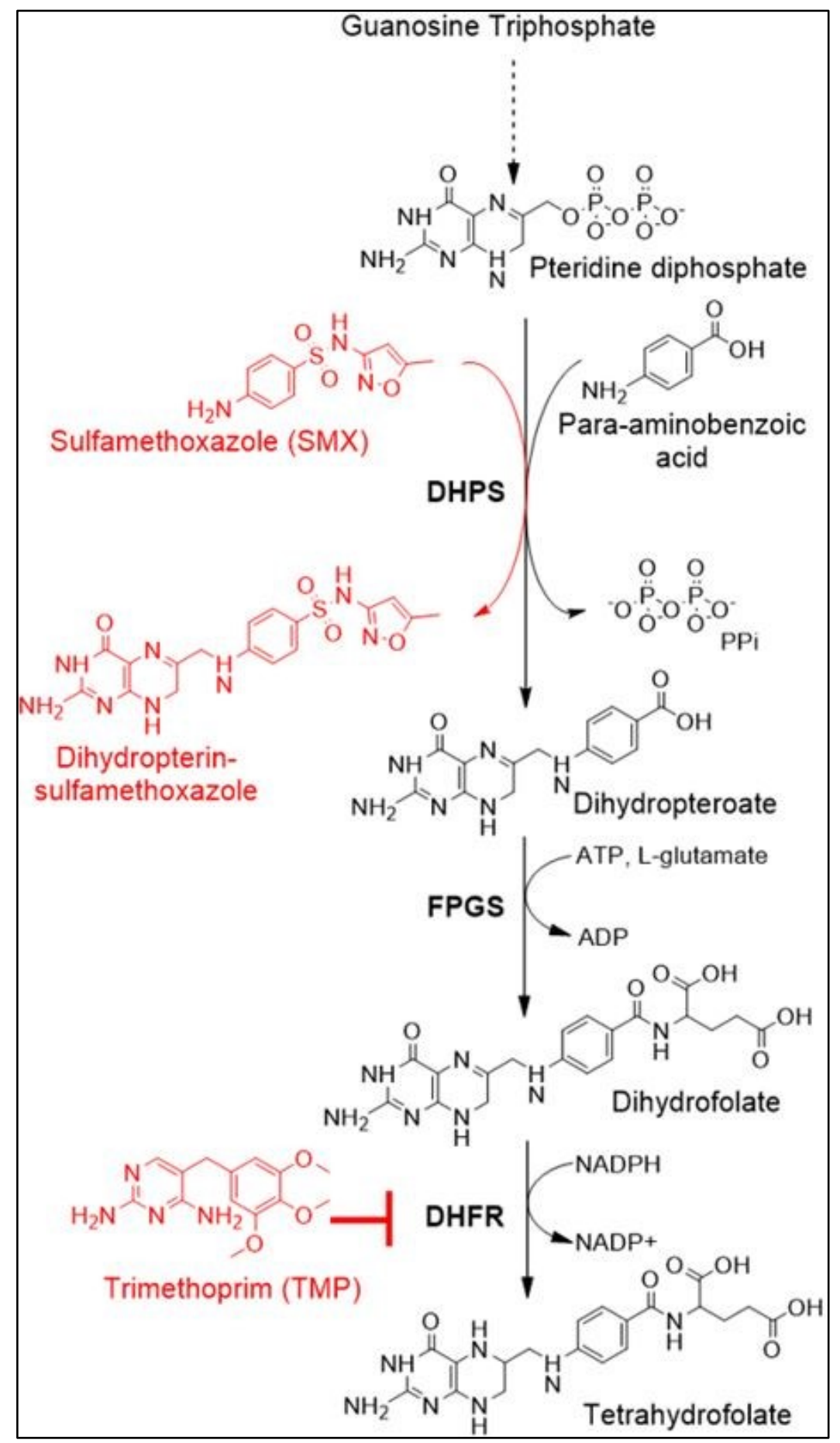

Figure 1-2. The folate biosynthesis pathway.

Dihydropteroate synthase; $\mathrm{PPi}=$ Pyrophosphate; FPGS = Folypolyglutamate synthase; DHFR $=$ Dihydrofolate reductase . 


\section{Antibiotic Discovery Towards Cysteine Biosynthesis}

In addition to the FBP, cysteine biosynthesis is another metabolic branch that is of interest for antibiotic discovery. Cysteine is one of the 20 proteinogenic amino acids and has several essential biological roles. The thiol groups between two cysteines on disparate regions in the primary amino acid chain can form a disulfide bond, contributing to important aspects of the tertiary structure of proteins. Cysteine residues play important roles in metal cofactor binding in several enzymes (Beinhert 2000, Kessler 2006). In prokaryotes, a key branch point occurs between the reductive sulfate assimilation pathway (RSAP) and cysteine biosynthesis, and as a result cysteine is a precursor for sulfur-containing molecules involved in redox homeostasis and anti-oxidative defense, including glutathione, mycothione, and trypanothione (Schnell 2015) (Figure 1-3).

Similar to the early stages of folate biosynthesis, cysteine biosynthesis in bacteria is unique from mammalian systems. Prokaryotic cysteine biosynthesis occurs in two distinct steps (Kredich 1966) (Figure 1-3). Serine acetyl transferase (SAT) catalyzes the first step, wherein OALS is synthesized from L-serine and acetyl CoA. In the second step, cysteine synthase A (CysK) produces L-cysteine from OALS and sulfide. In many bacterial species, the isoenzyme cysteine synthase $\mathrm{B}$ (CysM) is present but understood to only be active in anaerobic conditions (Kredich 1996).

The C-terminal tail of SAT inhibits the CysK active site in the absence of adequate pools of OALS (Huang 2005). Various inhibitor series have emerged that mimic the inhibitory interactions of SAT with the CysK enzyme (Campanini 2005, Salsi 2010). Isozyme-specific inhibitors of CysK and CysM from $S$. typhimurium have been discovered through virtual screening (Spyrakis 2013). Other inhibitor series targeting CysK have been developed for the treatment of Mycobacterium tuberculosis (Jean Kumar 2013, Poyraz 2013), Trichomoniasis vaginalis (Singh 2013, Westrop 2006) and Haemophilus influenzae (Salsi 2010, Amori 2012). Natural product derived naphthoquinone compounds inhibiting CysK have been discovered with amoebicidal activity against Entamoeba histolytica (Mori 2015).

Rather than inhibit CysK directly, an alternative mode of action against CysK was presented by Kredich and coauthors in the 1970s. It was found that the growth of $S$. typhimurium could be inhibited by formation of a false product mediated by CysK between 1,2,4-triazole and OALS (Kredich 1975). This led to dysregulation of the transcription of genes related to sulfur metabolism. This inhibitory mechanism does not appear to have been revisited since.

\section{Drug Discovery Challenges Unique to GN Bacteria}

A puzzling phenomenon occurring since the Golden Age of antibiotic discovery is the fact that antimicrobial drug discovery has waned despite incredible scientific advances in genetics, structural biology, and high throughput screening (HTS) (Silver 2011). The scientific literature from the past few decades is replete with exciting new 


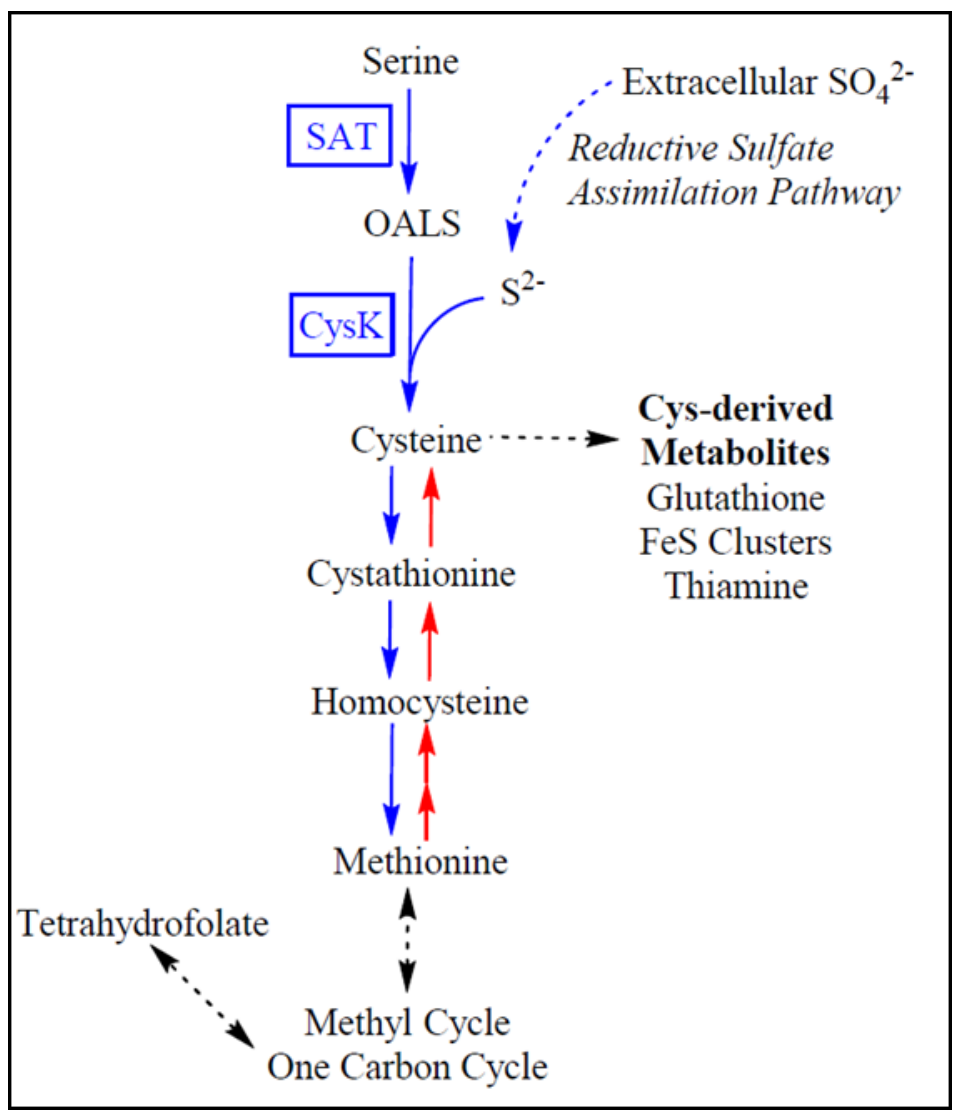

Figure 1-3. Cysteine biosynthesis and associated pathways in prokaryotes and mammals.

Blue lines represent metabolic processes unique to prokaryotes and lower eukaryotes, while red lines represent metabolic processes unique to higher eukaryotes. 
inhibitor series engaging novel bacterial targets, yet very few make it to the clinic. A great deal of time and resources are often devoted to the in vitro characterization and development of new inhibitor series, but these compounds perform poorly when introduced to whole cell assays. Formidable barriers to achieving biological activity are poor penetration of the bacterial cell membrane as well as limited residence time due to efflux and target modification (Figure 1-4). The unique physiological properties and sophisticated resistance mechanisms of the bacterial cell contribute to the high attrition rate associated with the anti-infective drug discovery process. The medicinal chemistry requirements for effective antibiotic drug design does not reliably follow parameters such as Lipinski's rule of five that have been useful for drug development against human targets (Lewis 2013).

The majority of the most concerning pathogenic species contributing to the current AMR crisis are GN bacteria, which are especially difficult to drug (Brown 2014, Silver 2016). GN bacteria have an additional outer membrane which distinguishes them from GP bacteria and further limits the entry of molecules with diverse physiochemical properties. If a molecule finds its way across the outer membrane, it must traverse the periplasmic space, penetrate the cytoplasmic membrane, and reside in the cytoplasm long enough to exert its intended antimicrobial effect. Molecules are susceptible to efflux and modification in both the periplasmic and cytoplasmic space (Figure 1-4). GN bacteria are especially notorious for their ability to efficiently efflux several antibiotic classes, limiting the residence time and efficacy of the drug (Silver 2016). Methods to gauge and refine the ability of early stage inhibitors to perform optimally in the GN cell system are certainly needed to reduce the high attrition rates observed in early stage inhibitor development.

\section{Improving Antibiotic Drug Discovery by Engaging Critical Metabolic Pathways}

Microorganisms are evolutionarily distinct from humans. From a cellular perspective, humans and microbes share similar requirements for basic cell metabolism. Prokaryotes and lower eukaryotes, however, have developed unique metabolic pathways to accommodate rapid changes in their environment, such as during the establishment of an infection. Higher eukaryotes have formed a reliance on their diet and commensal microbes to obtain essential metabolites. These factors have resulted in vast distinctions between humans and pathogenic microbes in terms of available biosynthetic machinery and metabolic requirements, suggesting bacterial metabolism as a promising avenue for antibiotic drug discovery and development. For these reasons, this dissertation examines bacterial metabolism as a source of potentially life-saving therapies with a focus on improving current methods in antibiotic drug discovery. 


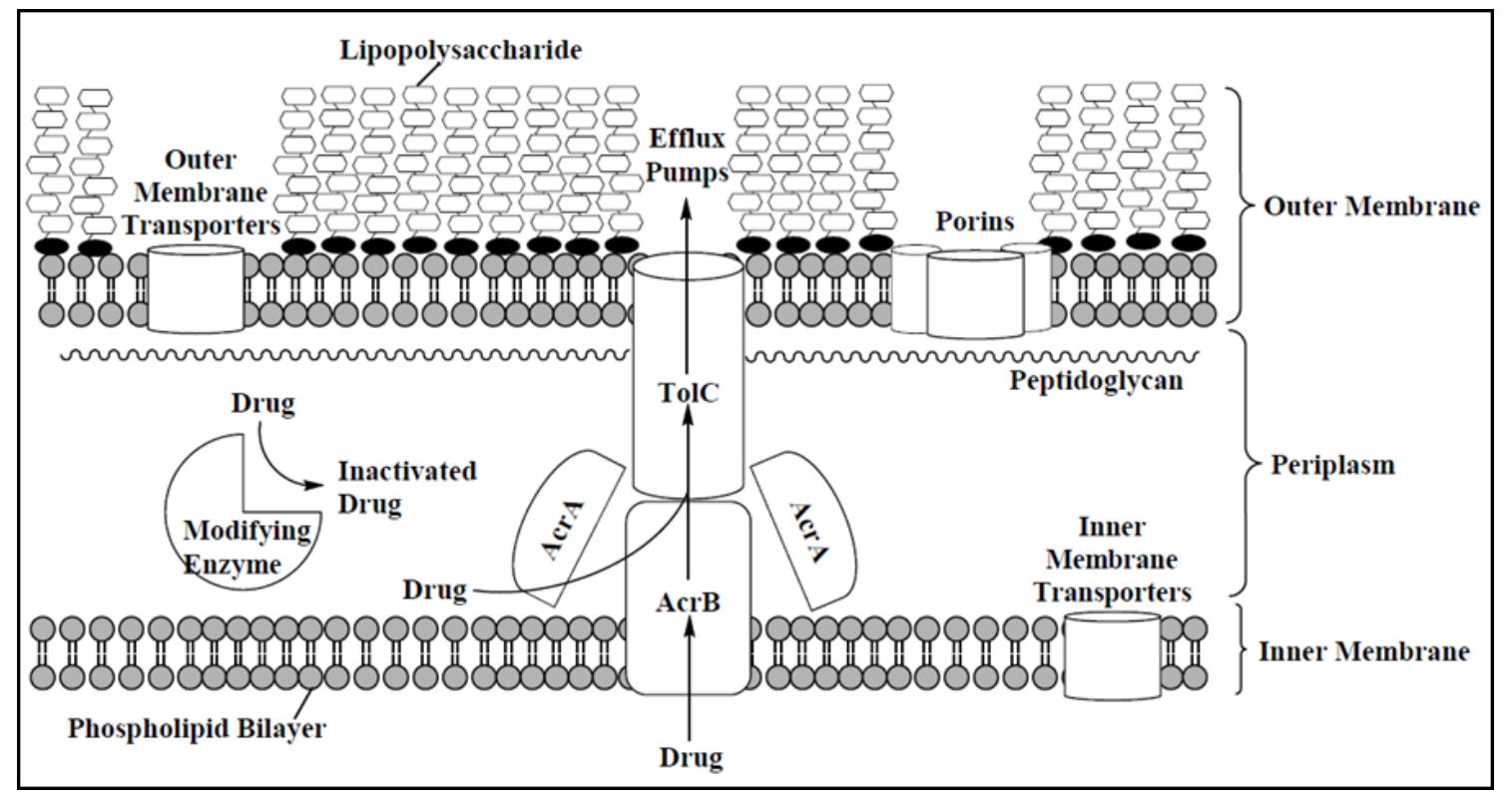

Figure 1-4. The Gram-negative cell membrane system.

The dual membrane system, efflux machinery, and modifying enzymes within the periplasm and cytoplasm can all have negative effects on drug entry and accumulation. 


\section{CHAPTER 2. ANALYSIS OF CHROMOSOMAL RESISTANCE MUTATIONS IN STAPHYLOCOCCUS AUREUS ${ }^{1}$}

\section{Introduction}

\section{Sulfonamide resistance in $S$. aureus and other clinically relevant species}

Sulfonamides are a cornerstone treatment option for a wide range of infection types and have had clinical utility for over 80 years. However, resistance to sulfonamides was observed very quickly after its discovery and is still reported (Domagk 1935, Bermingham 2000). Although the co-trimoxazole combination with TMP reduces the likelihood of resistance, global resistance to both SMX and TMP is increasingly observed and is a significant clinical issue today (Sköld 2000).

Resistance to sulfonamides can arise by a variety of mechanisms among pathogens. Several species including S. aureus, E. coli, Streptococcus pneumoniae, Plasmodium spp., and Pneumocystis spp. obtain sulfonamide resistance through chromosomal mutations in the target enzyme DHPS (Sköld 2000). Increased production of $p \mathrm{ABA}$ has also been observed in resistant strains of $S$. aureus, which has been shown in various studies to antagonize the growth inhibitory action of sulfonamides (Landy 1943, Zlitni 2013). GN enteric pathogens including E. coli, Klebsiella pneumoniae, and $S$. typhimurium acquire sulfonamide resistance horizontally through dissemination of sul genes 1, 2, and 3 which encode resistant DHPS isoenzymes (Toleman 2007).

Sulfonamide resistance in $S$. aureus is a particularly pressing issue due to the recent epidemic of MRSA infections, for which antifolates are a key second-line treatment option when other regimens fail (Zetola 2005). Globally, staphylococcal species are more frequently exhibiting resistance to multiple antibiotic therapies including vancomycin, daptomycin, and linezolid (Tacconelli 2018). Although cotrimoxazole is often relegated to the status of a second-line treatment option, it remains a valuable, cheap and effective regimen for multiple infection types caused by drug resistant staphylococci including skin and soft tissue infections and sepsis (Bowen 2017, Goldberg 2010). Therefore, antifolate resistance in staphylococcal species is a major concern due to the fact that these therapies serve as crucial treatment options, especially when frontline drugs fail.

\footnotetext{
${ }^{1}$ Modified from final submission with open-access permission. Griffith EC, Wallace MJ, Wu Y, Kumar G, Gajewski S, Jackson P, et al. The structural and functional basis for recurring sulfa drug resistance mutations in Staphylococcus aureus dihydropteroate synthase. Front Microbiol. 2018 Jul 17;9:1369. [doi:10.3389/fmicb.2018.01369]
} 


\section{Improved understanding of DHPS structure and function in recent decades}

Given the immense clinical utility of sulfonamides and their efficacy against $S$. aureus, next-generation DHPS inhibitors that are both effective against $S$. aureus and recalcitrant to known sulfonamide resistance mechanisms are highly desired Bermingham 2000). Effective drug development regimes benefit from a comprehensive understanding of the structure and function of the inhibitor target. Fortunately, several studies over the last couple of decades have vastly improved the understanding of the DHPS structure and catalytic mechanism as well as the overall FBP. The structures of DHPS have been reported for several pathogenic species including $S$. aureus (Hampele 1997), B. anthracis (Babaoglu 2004), Yersinia pestis (Yun 2012), S. pneumoniae (Levy 2008), Francisella tularensis (Shaw 2014), and M. tuberculosis (Baca 2000). DHPS is a dimeric ( $\beta / \alpha)$ TIMbarrel enzyme with the active site situated at the $\mathrm{C}$-terminal end of the barrel (Figure 21A). The active site consists of three regions: A structured pterin binding site deep within the TIM barrel, an anion binding pocket occupied by the DHPP leaving group pyrophosphate (PPi), and a set of flexible loops at the C-terminal edge of the enzyme where both $p$ ABA and sulfonamides bind. Sulfonamides are structural mimetics of the native substrate $p$ ABA. They bind in the same flexible loop region and undergo DHPSmediated catalysis to form a metabolically useless false product with DHPP (Palmer 2014).

DHPS native catalysis was originally hypothesized to occur by a $\mathrm{S}_{\mathrm{N}} 2$ mechanism (Baca 2000), but more recent studies indicate the reaction is more likely a $S_{N} 1$ reaction, wherein a cationic intermediate is formed by release of PPi before the nucleophilic attack of $p \mathrm{ABA}$ on the $\mathrm{DHP}+$ intermediate, forming the 7,8-dihydropteroate product (Yun 2012) (Figure 2-1B). The $p \mathrm{ABA} /$ sulfonamide binding site at the $\mathrm{C}$-terminal edge of the DHPS enzyme is composed of a disordered flexible loop system that has proven difficult for structural characterization. A 2012 study on $Y$. pestis DHPS successfully captured the conformations of loops 1 and 2 which form a substructure during native catalysis necessary to form the $p$ ABA binding pocket (Yun 2012). These two loops are well conserved among multiple species and sulfonamide resistance mutations are commonly observed in this region among several pathogenic species (Babaoglu 2004). The same study on $Y$. pestis DHPS also revealed functional roles for the magnesium cofactor in aiding the release of PPi and helping to order the loop 1-2 substructure (Yun 2012).

Recent structural and functional characterizations have also revealed potential new sites for inhibitor binding in addition to the traditional sulfonamide binding site which is amenable to mutation. Allosteric inhibitors that bind the DHPS dimer interface have been discovered and characterized. These inhibitors prevent "breathing motions" within the DHPS dimer that normally aid in substrate release from the enzyme and influence the loop 1-2 active site structure (Hammoudeh 2014). The pterin binding pocket has also been investigated as a potential site for inhibitor engagement. Monocyclic pteridine analogs were developed by Burroughs Wellcome in the 1980s to engage the pterin binding site of DHPS (Lever 1985). Structure-based technologies allowed for improving the engagement of the pterin site with pyridazine inhibitors (Zhao 2012). This study also garnered an improved understanding of interactions of both native 


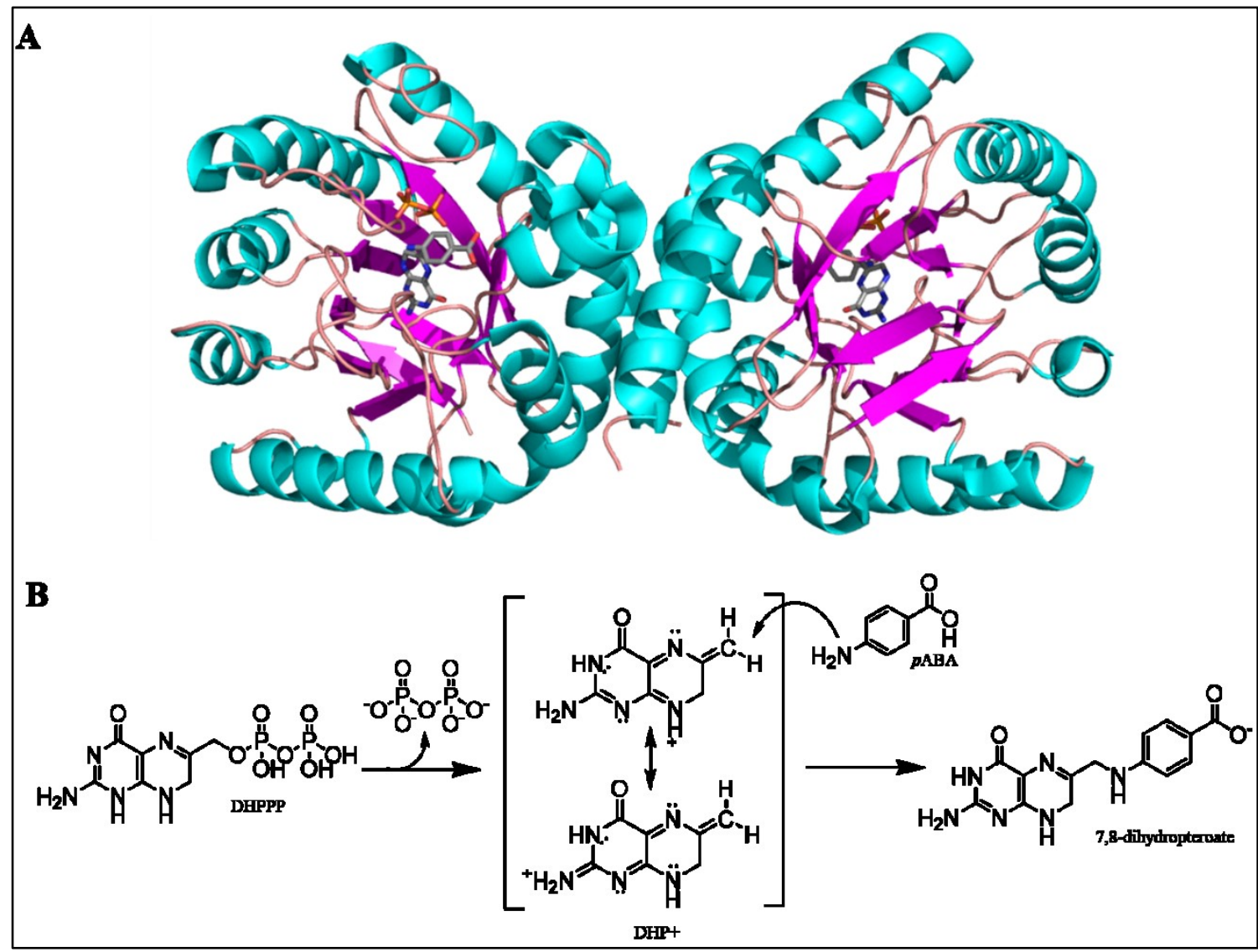

Figure 2-1. Structure and catalytic mechanism of DHPS.

A. DHPS transition state complex structure from PDB 3TYZ with three ligands bound including the $\mathrm{DHP}+$ cationic intermediate, $\mathrm{PPi}$, and $p \mathrm{ABA}$. $\mathrm{DHP}+$ and $p \mathrm{ABA}$ are in grey sticks, while PPi is in orange sticks. B. $\mathrm{S}_{\mathrm{N}} 1$ catalytic mechanism carried out by DHPS. 
substrate DHPP and inhibitors with conserved residues comprising this pterin binding site.

\section{Recent insights into the FBP function and candidacy for next-generation inhibitor development}

Folate biosynthesis is highly interconnected with diverse branches of bacterial metabolism. Both the products and intermediates of this pathway have important roles in other biosynthetic pathways and basic cell functions (Bermingham 2002). The pathway begins with GTP, which is a purine nucleobase that is well known for its role as a building block of RNA during transcription. The DHPS substrate $p$ ABA is derived from chorismate, which is also a precursor for aromatic amino acids and other aromatic metabolites (Dosselaere 2001). Folates and derivatives have established roles in both the folate cycle and one-carbon metabolism, which is responsible for methylation reactions, thymidylate biosynthesis, and the biosynthesis of glycine, serine, and methionine. THF is also a precursor for inosine-monophosphate, pantothenate, and $\mathrm{RRNA}^{\mathrm{fmet}}$ in addition to other important metabolites (Bermingham 2002).

Due to the interconnectedness of both the enzymes within the FBP and other closely associated metabolic pathways, the synergy and efficacy of antifolates in combination therapy is not surprising. For several decades, however, the detailed mechanism of synergy between sulfonamides and TMP was not extensively delineated. The fact that DHPS precludes DHFR in the pathway suggests that sulfonamides potentiate TMP by limiting the DHFR substrate DHF, but the mechanism of potentiation of sulfonamides by TMP was less obvious. A recent study identified a metabolic feedback cycle between THF and DHPP. Knocking out enzymes within this loop increased susceptibility to SMX (Minato 2018). This suggests a mechanism wherein TMP potentiates SMX by limiting the production of inosine-monophosphate, which is a precursor for GTP and consequently also a precursor for DHPP.

In recent years the role of $p \mathrm{ABA}$ as both an antagonist of sulfonamides as well as a potential new antibiotic target has been explored. The growth inhibitory actions of various antibiotic classes are known to be antagonized by metabolites that are closely related to the target protein, and supplementation with $p$ ABA has been shown to mask the growth inhibitory action of sulfonamides (Zlitni 2013). Disrupting the biosynthesis of $p$ ABA by small molecule inhibitors is an emerging area of interest in inhibiting the growth of both E. coli and M. tuberculosis. Limiting $p$ ABA production has also been shown to potentiate the effects of antifolates (Zlitni 2013, Thiede 2016).

The efficacy of antifolates is clearly due to the important roles assumed by folates in several essential aspects of bacterial metabolism. This pathway remains an attractive target for new inhibitor development, and DHPS is an excellent candidate for new drug design due to improved understanding of both the structure and function of the enzyme as well as potential new druggable sites (Bermingham 2002). To achieve effective inhibitor 
development, progress towards understanding resistance mechanisms from a structural and functional standpoint is still required and is addressed within this chapter.

\section{Materials and Methods}

\section{Genetic survey of available $S$. aureus DHPS sequences}

The genetic survey of DHPS sequence variations from $S$. aureus was conducted in 2014. The DHPS WT sequence from Rosenbach 25923 was entered into a GenBank BLAST search among the available genetic data for $S$. aureus. A set of 80 non-redundant DHPS sequences and 56 reference strains were retrieved and compared to the WT DHPS sequence. The sequences were categorized based on having a 25923 or 8325 background as well as having known mutations associated with resistance.

\section{Heterologous expression and purification of recombinant DHPS variants}

The gene encoding folP from S. aureus 25923 (EMBL Z84573) and the genes representing each mutation alone and in combination were codon optimized for expression in the E. coli host and cloned into the pET16b vector with an N-terminal 10x Histidine tag. The gene for folP from $S$. aureus 8325 was also cloned into pET16b in this manner. All plasmids were transformed into E. coli BL21[DE3] cells. Transformants that grew on the selective agent ampicillin were selected and sequenced to ensure successful transformation.

For purification of the DHPS variants, an overnight culture of the transformed $E$. coli was used to inoculate 4 liters of LB with ampicillin at at $50 \mu \mathrm{g} / \mathrm{mL}$. Once the cultures reached mid-log $\left(\mathrm{OD}_{600}\right.$ between 0.4 and 0.7$)$, IPTG was added to a final

concentration of $1 \mathrm{mM}$, and the cultures were allowed to incubate overnight at $18^{\circ} \mathrm{C}$ with 225 RPM shaking. The next day, the cultures were centrifuged at $3700 x \mathrm{x}$ for 10 minutes, the supernatants were removed, and the pellets were kept on ice. The buffers used for lysis and purification were "Buffer A," which contained $50 \mathrm{mM}$ Tris-HCl, 500 $\mathrm{mM} \mathrm{NaCl}, 5 \mathrm{mM}$ imidazole, $\mathrm{pH}$ 8, and "Buffer B" which was the same except for having $500 \mathrm{mM}$ imidazole. $50 \mathrm{~mL}$ buffer A with a protease inhibitor cocktail tablet (Roche 11836-170-001) and $500 \mathrm{mg}$ lysozyme were used to resuspend each 1 liter pellet by pipetting and vortexing. The lysates then underwent sonication at 3 minutes and 100\% amplitude three times. An additional 3 minutes was added to each sonication time for each additional liter of bacteria. The lysates were then centrifuged at 20,000 RCF for 30 minutes at $4^{\circ} \mathrm{C}$. The resulting supernatants were passed through a $0.22 \mu \mathrm{M}$ filter.

The prepared lysates were loaded onto a GE HisTrap HP $5 \mathrm{~mL}$ column at 0.5 $\mathrm{mL} / \mathrm{minute}$ to allow slow binding of the Histidine-tagged DHPS proteins onto the column. Buffer A was allowed to wash the column for several column volumes to remove background proteins. DHPS protein was then eluted in $1 \mathrm{~mL}$ fractions by a 0 - 
$100 \%$ Buffer B gradient over a span of two hours at $0.5 \mathrm{~mL} / \mathrm{min}$. Fractions with an elevated UV spectra were analyzed by total protein staining after SDS-PAGE electrophoresis to identify fractions with purified DHPS at the expected molecular weight. Fractions containing DHPS were then pooled and run over a HiPrep 16/60 Sephacryl S-200 HR column at $0.5 \mathrm{~mL} / \mathrm{min}$ in $50 \mathrm{mM}$ HEPES, $150 \mathrm{mM} \mathrm{NaCl}, 1 \mathrm{mM}$ DTT, $\mathrm{pH} 7.6$ and fractionated as before. The fractions were again analyzed by SDSPAGE electrophoresis. Fractions containing at least 95\% pure DHPS were retained and pooled. The protein was then flash-frozen in liquid Nitrogen and stored at $-80^{\circ} \mathrm{C}$ for up to six months.

\section{Generation of isogenic $S$. aureus strains}

The folP gene from $S$. aureus strain NCTC 8325 was ligated into the PCR2.1TOPO plasmid. The eight mutants of this gene, including F17L, S18L, T51M, E208K, KE257_dup, F17L E208K, F17L KE257_dup, and T51M E108K, were introduced to the folP gene via site-directed mutagenesis. These genes were then subcloned into the PJB38 vector, which has an S. aureus origin of replication that is temperature sensitive. These plasmids were transformed into $S$. aureus USA300 AH1263. Transformants were allowed to grow in media containing CAM at $43^{\circ} \mathrm{C}$ to allow for plasmid integration. The bacteria were then grown at $30^{\circ} \mathrm{C}$ and grown on anhydrous tetracycline to allow for killing of cells that still retained the plasmid. The successful transformants were further confirmed by sequencing of the chromosomal folP gene and testing for susceptibility to CAM. Except for T51M E208K, all other mutants were successfully cloned.

\section{Minimum inhibitory concentration (MIC) determination}

All MIC testing was performed in SSM9PR media, which is a minimal media developed for $S$. aureus (Reed 2015). The drugs of interest were diluted two-fold across 96-well plates in a total volume of $100 \mu \mathrm{L}$ per well. Colonies of each strain tested were used to inoculate a small volume of SSM9PR media to a mid-log OD 600 between 0.3 and 0.7. This culture was further diluted to an $\mathrm{OD}_{600}$ of 0.001 . To each well of the drugged MIC plate, $100 \mu \mathrm{L}$ of the diluted culture was added, allowing for approximately $10^{5} \mathrm{CFU}$ to be added per well. The plates were allowed to incubate at $37^{\circ} \mathrm{C}$ for 16 hours, after which the $\mathrm{MIC}_{80}$ was determined visually. Each MIC reported is an average of three biological replicates, which were each performed on different days.

\section{Growth rate study}

A single colony of each mutant was used to inoculate $5 \mathrm{~mL} \mathrm{LB}$ broth and grown overnight at $37^{\circ} \mathrm{C}, 225 \mathrm{RPM}$ shaking. The next day, the cultures were diluted 1:100 and the $\mathrm{OD}_{600}$ was read every 30 minutes. Using data from the exponential phase for each 
strain, the doubling times for each mutant was calculated following previously described methods (Reeve 2016):

\section{Wax moth larvae infection study}

Galleria mellonella (wax moth) larvae in the final instar stage (Fisher Scientific 14-726-369) were stored at $13^{\circ} \mathrm{C}$ and used within a week of receipt. For each experiment, all larvae came from the same batch. Each group contained 32 larvae, which were stored in square bioassay dishes lined with filter paper. Control groups for each experiment always included a group that was not manipulated as well as a group that was only injected with the vehicle.

For the virulence and rescue studies, the inoculum was prepared by first inoculating $5 \mathrm{~mL}$ of LB with a single colony of the strain tested. Once this culture reached mid-log, it was washed with ice-cold PBS and further diluted 1:20 in ice-cold PBS. Each larvae was administered an intrahaemocoelic injection with $10 \mu \mathrm{L}$ of the prepared inoculum, allowing for $5.9 \times 10^{5} \mathrm{CFU}$ to be administered. The inoculums were enumerated in triplicate to ensure appropriate and equal bacterial densities among all strains tested. For the rescue experiments, $10 \mu \mathrm{L}$ of SMX in a PBS $/ 5 \%$ DMSO solution was administered two hours before the infection and at 24 and 48 hours at a final concentration of $100 \mathrm{mg} / \mathrm{kg}$ in each larvae. Larvae were monitored periodically to determine mortality rates. Mortality was determined if both a lack of motility and change in color were observed (Tsai 2016). To determine significant differences in mortality rates among the groups tested, the Log-rank (Mantel-Cox) test was employed.

\section{X-ray crystallography}

Purified SaDHPS F17L E208K was equilibrated and concentrated to $20 \mathrm{mg} / \mathrm{mL}$ in $50 \mathrm{mM}$ HEPES, $150 \mathrm{mM} \mathrm{NaCl}$, and $1 \mathrm{mM}$ DTT at $\mathrm{pH}$ 7.6. Crystallization optimization trials yielded a favorable condition at $18^{\circ} \mathrm{C}$ of $0.2 \mathrm{M}$ sodium nitrate, $25 \mathrm{mM}$ HEPES, 75 $\mathrm{mM} \mathrm{NaCl}, 20 \%$ PEG 3350, pH 7.6. For the co-crystal structure, 1530 was added at a 3:1 molar ratio with the enzyme and soaked for 16 hours. The soaked crystals were combined with a cryoprotectant of $25 \%$ glycerol and flash-frozen. Data was collected using the 22-ID X-ray beamline at the Southeast Regional Collaborative Access Team (SER-CAT) at Argonne National Laboratory. XDS was used to index and integrate diffraction images (Kabsch 2010). The images were further scaled and merged using CCP aimless in the P43 space group. PHASER was used to solve the structure using coordinates from SaDHPS PDB 1AD4 as a search model (Hampele 1997, McCoy 2007). PHENIX was employed for reciprocal space refinement with NCS restraints, twin law h,$\mathrm{k},-1$, and TLS definitions within final cycles (Afonine 2012). To create ligand restraints, eLBOW was employed (Moriarty 2009). COOT was used for real space refinement (Emsley 2004). The structures have been deposited in the Protein Data Bank with the accession codes 6CLU and 6CLV. Refinement statistics and data collection data are previously reported (Griffith and Wallace, 2018). 


\section{Results and Discussion}

\section{Identification of five sulfonamide resistance mutations in S. aureus DHPS}

Over the last couple of decades, improvements in sequencing and deposition of genetic information on clinical isolates has provided ample information in GenBank to identify variations in the folP gene of $S$. aureus, which encodes DHPS. 132 DHPS sequences were identified in GenBank including sequences from 56 reference strains and 80 non-redundant DHPS sequences. Among these, 16 variations were present in the DHPS enzyme (Table 2-1).

In a 1997 study by Hampele and coauthors, 15 of these variations were observed among a collection of sulfonamide-resistant clinical isolates, leading the authors to conclude the mechanism of DHPS resistance to be due to mutations in combination (Hampele 1997). It was noted that both $S$. aureus Rosenbach 25923 and NCTC 8325 are sulfonamide susceptible, and there are 11 variations between the two strains. Therefore, these variations were considered natural polymorphisms or background mutations that have no role in resistance (Table 2-1).

This left five mutations, F17L, S18L, T51M, E208K and KE257_dup. All of these mutations apart from S18L were identified in the previous study on $S$. aureus DHPS, and the isolates in that study exhibited high level sulfonamide resistance (Hampele 1997). Furthermore, previous studies in our lab revealed that SMX-resistant $S$. aureus harboring the S18L mutation could be obtained upon serially passaging wild-type (WT) S. aureus in increasing concentrations of SMX (Griffith 2015). Genetic data from both the available GenBank sequences and the Hampele et al. study revealed that F17L, S18L, and T51M mutations could be observed by themselves while the E208K and KE257_dup mutations were only observed in combination. This led to the categorization of these mutations as primary and secondary, respectively (Tables 2-1 and 2-2).

\section{Identification of homologous resistance mutations in other species}

In addition to $S$. aureus, sulfonamide resistance is known to occur in several other diverse species of microorganisms. Therefore, this study on resistance mutations in the DHPS enzyme of $S$. aureus could also inform resistance mechanisms in other clinically relevant species. Towards this end, the available literature on sulfonamide resistance was surveyed. Mutations homologous to the identified sulfonamide resistance mutations in $S$. aureus were discovered that originated either in in a lab setting or in clinical isolates that were exposed to the drug in vivo (Table 2-3). The F17L mutation in loop 1 was identified in E. coli (Dallas 1992), B. anthracis (Yun 2012), and Neisseria meningitidis (Fermer 1995). Mutations homologous to T51M in loop 2 were identified in S. pneumoniae (Maskell 1997), Pneumocystis carinii, Plasmodium spp. (Mei 1998, Pornthanakasem 2016), and M. leprae (Kai 1999). Two Plasmodium species, P. falciparum and P. vivax, also had a mutation homologous to the E208K secondary mutation (Pornthanakasem 
Table 2-1. Categorization of DHPS amino acid variations identified in this study.

\begin{tabular}{ll}
\hline Mutation Type & \multicolumn{1}{c}{ Mutation } \\
\hline Background $^{\mathrm{a}}$ & $\begin{array}{l}\text { I30V, N31T, I37M, V58I, S59T, } \\
\text { L60V, M64L, M101I, I117V, } \\
\text { I126V, L266F }\end{array}$ \\
$\begin{array}{l}\text { Primary } \\
\text { Resistance }\end{array}$ & F17L, S18L, T51M \\
$\begin{array}{l}\text { Secondary } \\
\text { Resistance }\end{array}$ & E208K, KE257_dup \\
\hline
\end{tabular}

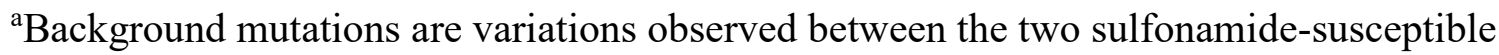
S. aureus strains NCTC 8325 and Rosenbach 25923.

${ }^{\mathrm{b}} \mathrm{KE} 257$ dup is a KE duplication insertion mutation at position 257.

Table 2-2. Survey of DHPS sequence variations and reported sulfonamide susceptibility.

\begin{tabular}{|c|c|c|c|c|c|c|c|c|c|c|}
\hline $\begin{array}{l}\text { Sequence } \\
\text { Background }\end{array}$ & 25923 & 8325 & 8325 & 25923 & 8325 & 8325 & 25923 & 8325 & 25923 & 8325 \\
\hline $\begin{array}{l}\text { Resistance } \\
\text { mutations }\end{array}$ & None & None & $\begin{array}{c}\text { F17L } \\
\text { KE257_dup }\end{array}$ & $\begin{array}{c}\text { T51M } \\
\text { E208K }\end{array}$ & $\begin{array}{r}\text { T51M } \\
\text { E208K }\end{array}$ & $\begin{array}{c}\text { F17L } \\
\text { E208K }\end{array}$ & F17L & F17L & $\mathrm{T} 51 \mathrm{M}$ & $\mathrm{S} 18 \mathrm{~L}$ \\
\hline $\begin{array}{l}\text { \% Sequences } \\
(\mathrm{n}=136)\end{array}$ & 28 & 49 & 3 & 8 & 1.5 & 3.7 & 3 & 2 & 0.7 & 0.7 \\
\hline $\begin{array}{l}\text { Hampele MIC } \\
(\mu \mathrm{g} / \mathrm{mL})^{\mathrm{a}}\end{array}$ & & & $256->1024$ & $\begin{array}{c}256- \\
>1024\end{array}$ & $>1024$ & $>1024$ & & & & \\
\hline $\begin{array}{l}\text { Sulfonamide } \\
\text { resistant }\end{array}$ & No & No & Yes & Yes & Yes & Yes & ND & ND & ND & ND \\
\hline
\end{tabular}

${ }^{a}$ Data source: MIC values from Hampele IC, D'Arcy A, Dale GE, Kostrewa D, Nielsen J, Oefner C, et al. Structure and function of the dihydropteroate synthase from Staphylococcus aureus. J Mol Biol. 1997 Apr 25;268(1):21-30. 
Table 2-3. Occurrence of identified sulfonamide resistance mutations in other pathogenic species.

\begin{tabular}{|c|c|c|c|}
\hline Classification & Mutation & Location & $\begin{array}{c}\text { Similar Mutations in Other } \\
\text { Organisms }\end{array}$ \\
\hline \multirow[t]{3}{*}{ Primary } & F17L & Loop 1 & \multirow{3}{*}{$\begin{array}{l}\text { E. coli, N. meningitidis, B. anthracis } \\
\text { None } \\
\text { S. pneumoniae }{ }^{a}, P \text {. falciparum }{ }^{a}, P \text {. } \\
\text { vivax }^{a}, \text { M. leprae, P. carinii }\end{array}$} \\
\hline & S18L & Loop 1 & \\
\hline & T51M & Loop 2 & \\
\hline Secondary & $\begin{array}{l}\text { E208K } \\
\text { KE257 dup }\end{array}$ & $\begin{array}{l}\alpha \text {-helix Loop } 7 \\
\alpha \text {-helix } 8\end{array}$ & $\begin{array}{l}\text { P. falciparum } \\
\text { None }\end{array}$ \\
\hline
\end{tabular}

a b Indicates that T51M and E208K mutations align with observed mutations in these species, but these mutations are not strictly conserved. 
2016). Mutations corresponding to S18L and KE257_dup were not identified in other species.

An alignment was assembled to compare the DHPS primary sequences of $S$. aureus and other pathogenic species (Figure 2-2). It was observed that amino acids within loops 1 and 2 are highly conserved, and the observed primary mutations F17L, S18L, and T51M reside within these highly conserved regions. The two secondary mutations, E208K in $\alpha$-helix loop 7 and KE257_dup at the dimer interface, are not conserved among the various microbial species studied and thus appear to represent sulfonamide resistance mutations unique to $S$. aureus.

\section{In vitro characterization of DHPS enzymes harboring sulfonamide resistance mutations}

The location of primary mutations in conserved regions of the enzyme with known roles in native catalysis indicates mutations may have consequences in terms of biochemical function. The secondary mutations were never identified without the presence of a primary mutation and occur on sites of the DHPS enzyme that are less conserved without an immediately obvious role in enzymatic function. These observations suggest that the secondary mutations may compensate for fitness costs associated with the primary mutations which may affect the normal function of the enzyme.

To gain a clearer understanding of the consequence of each mutation on enzymatic function, nine total DHPS proteins harboring each mutation either singly or in the observed clinical combination were expressed and purified in a heterologous $E$. coli expression system. A series of biochemical experiments were performed using these DHPS variants (Table 2-4). A colorimetric assay was used to obtain $\mathrm{K}_{M}$ values for $\mathrm{SMX}, p \mathrm{ABA}$, and DHPP by tracking PPi that is released during the normal catalysis of the enzyme. Furthermore, $\mathrm{K}_{\mathrm{i}}$ values were obtained by an assay monitoring product formation between ${ }^{14} \mathrm{C}$-labeled $p \mathrm{ABA}$ and $\mathrm{DHPP}$.

It was found that primary mutations had a high consequence to the binding affinity for $p \mathrm{ABA}$ in contrast to DHPP. However, secondary mutations modulated the $\mathrm{K}_{\mathrm{M}}$ values for DHPP more so than $p \mathrm{ABA}$. This may be due to the proximity of primary mutations to the $p \mathrm{ABA}$ binding site, whilst secondary mutations are in more structurally sound regions of the enzyme that may impact the pterin binding region which is deep in the $\beta$-barrel. Mutations in combination consistently restored the $p \mathrm{ABA} \mathrm{K}_{\mathrm{M}}$ when compared to primary mutations alone, suggesting the secondary mutations do indeed have a compensatory effect (Table 2-4).

The two primary mutations F17L and S18L greatly increased both the binding affinity and inhibition constant for SMX. Addition of E208K even further increased this in vitro resistance. The impact of T51M on inhibition was less pronounced, even in combination with E208K. Substrate turnover for both $p$ ABA and SMX was also 


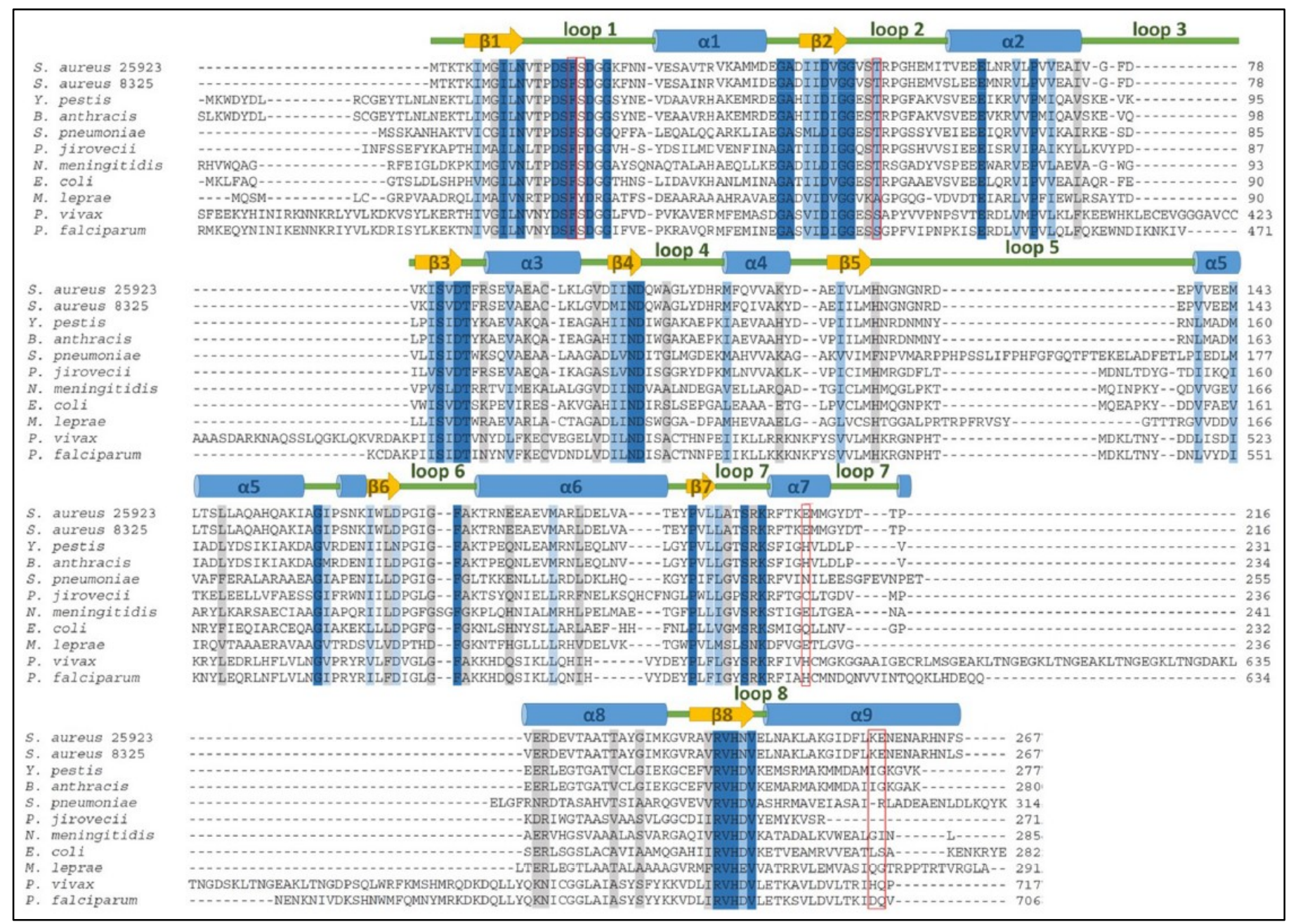

Figure 2-2. Sequence comparison of DHPS from $S$. aureus and other key pathogenic species.

Blue shading indicates $100 \%$ amino acid conservation, light blue indicates high conservation, and grey indicates weak conservation. The locations of identified resistance mutations are boxed in red. 
Table 2-4. Biochemical profile of SaDHPS variant enzymes.

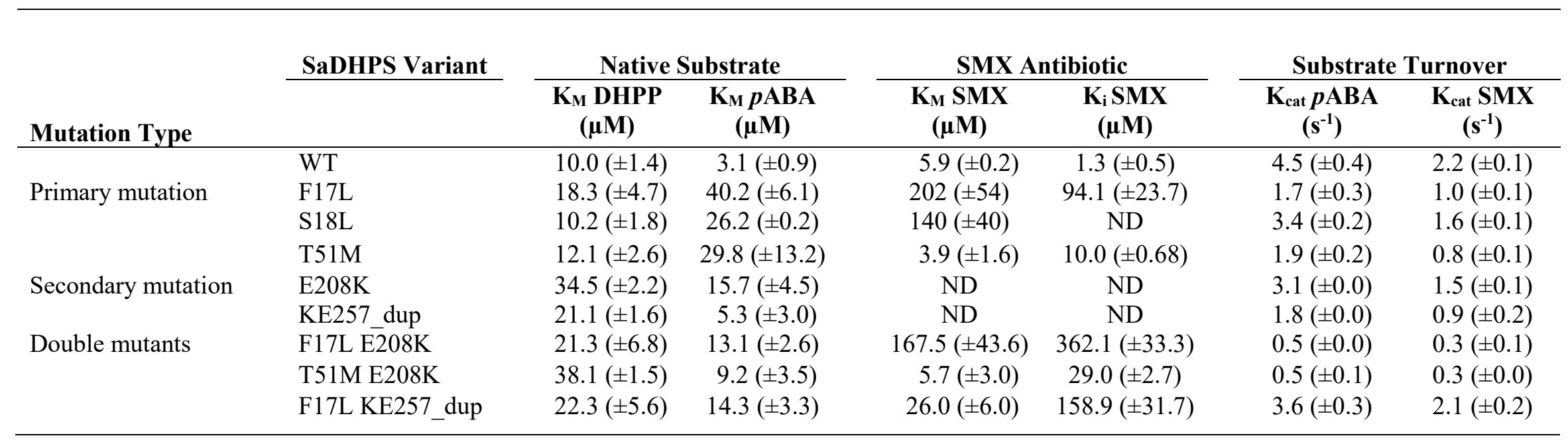


measured, and it was found that the mutations generally decrease turnover two-fold with an even further decrease in turnover with the E208K combinations (Table 2-4).

\section{Resistance profile of an $S$. aureus isogenic DHPS mutant panel against sulfonamide drugs}

To study the five DHPS mutations directly associated with sulfonamide resistance from a whole cell standpoint, a set of eight isogenic $S$. aureus DHPS mutants were generated in the USA300 AH1263 strain background. These isogenic strains harbored both the primary and secondary mutations either as single mutations or in their clinical combination (Tables 2-1 and 2-2). All mutants were successfully cloned with the exception of the T51M E208K combination. The $S$. aureus COL strain, which naturally harbors the T51M E208K set, was included for select experiments. The susceptibility of this panel of isogenic strains was tested against a variety of sulfonamide drugs (Table 2-

5). Chloramphenicol (CAM) was included as a control since its MOA is distinct from the antifolates. To avoid interference of metabolites common in rich testing medias with minimal inhibitory concentration (MIC) determination, MICs were performed in the SSM9PR minimal media which lacks $p$ ABA and various folate derivatives (Reed 2015).

All strains tested had very similar CAM MICs (Table 2-5). Among the primary mutants, F17L generally showed the most marked increases in sulfonamide MICs compared to the WT strain, with at least a two-fold MIC shift for seven of the ten sulfonamides tested. S18L and T51M showed less dramatic increases in susceptibility. Among all of the sulfonamides tested, the T51M single mutant only showed an MIC shift greater than two-fold for dapsone. In the case of T51M, this is notable due to the corresponding mutation being observed in M. leprae, Pneumocystis spp., and Plasmodium spp. In patients with sulfa allergies, dapsone is a common prophylactic treatment option in combination with TMP for Pneumocystis pneumonia in immunocompromised patients (Hughes 1998). Dapsone is also used in the treatment of both leprosy and malaria (Saha 2003). The use of dapsone to treat infections in these species likely encouraged this loop 2 mutation, which appears to have specific effects towards dapsone.

The two secondary mutations, E208K and KE257_dup, are located in less conserved regions of the enzyme that are more disparate from the active site locale. Therefore, their roles in resistance are unclear. Secondary mutations do not always show resistance on their own but may compensate for a fitness cost associated with primary mutations (Sander 2002). However, both DHPS secondary mutations alone showed moderate increases in MIC for many of the sulfonamides tested, indicating they have direct contributions to resistance as opposed to strictly serving as compensatory mutations (Table 2-5).

The susceptibility profile of strains with mutations in combination showed the strongest increases in resistance among the entire mutant panel. In the case of the combination mutations in the isogenic set (F17L E208K and F17L KE257_dup), the 
Table 2-5. MIC80 Values for $S$. aureus Isogenic DHPS Mutant Panel $(\mu \mathrm{g} / \mathrm{mL})$.

\begin{tabular}{|c|c|c|c|c|c|c|c|c|c|}
\hline \multirow[b]{2}{*}{ Compound } & \multirow{2}{*}{$\begin{array}{c}\begin{array}{c}\text { No } \\
\text { Mutation }\end{array} \\
\text { WT } \\
\end{array}$} & \multicolumn{3}{|c|}{ Primary Mutation } & \multicolumn{2}{|c|}{ Secondary Mutation } & \multicolumn{3}{|c|}{ Combination } \\
\hline & & F17L & S18L & T51M & E208K & KE257_dup & $\begin{array}{c}\text { F17L } \\
\text { E208K }\end{array}$ & $\begin{array}{c}\text { F17L } \\
\text { KE257_dup }\end{array}$ & $\begin{array}{c}\text { T51M } \\
\text { E208K }^{\mathrm{a}}\end{array}$ \\
\hline Sulfathiazole & 2.6 & 12.5 & 4.2 & 3.1 & 8.3 & 8.3 & 41.7 & 41.7 & 83.3 \\
\hline Sulfamethoxazole & 4.2 & 16.7 & 10.4 & 6.3 & 8.3 & 8.3 & 50.0 & 41.7 & 66.7 \\
\hline Sulfisoxazole & 2.1 & 6.3 & 5.2 & 2.1 & 6.3 & 6.3 & 25.0 & 25.0 & 58.0 \\
\hline Sulfadiazine & 10.4 & 25.0 & 25.0 & 8.3 & 20.8 & 20.8 & 83.3 & 83.3 & 200.0 \\
\hline Dapsone & 16.7 & 20.8 & 6.3 & 50.0 & 33.3 & 29.2 & 50.0 & 66.7 & $200->200$ \\
\hline Sulfamethoxypyridazine & 7.3 & 25.0 & 25.0 & 7.3 & 12.5 & 16.7 & 83.3 & 83.3 & 133.3 \\
\hline Sulfadimethoxine & 2.6 & 12.5 & 4.2 & 3.6 & 8.3 & 8.3 & 33.3 & 25.0 & 66.7 \\
\hline Sulfadoxine & 25.0 & 33.3 & 41.7 & 12.5 & 50.0 & 41.7 & 200.0 & 83.3 & $200->200$ \\
\hline Chloramphenicol & 4.2 & 4.2 & 3.1 & 3.1 & 3.1 & 3.1 & 3.1 & 3.1 & 3.1 \\
\hline $\mathrm{CMY} / \mathrm{TMPb}$ & $0.39 /$ & $0.78 /$ & $0.33 /$ & $0.39 /$ & $0.52 /$ & $0.65 /$ & $1.6 /$ & $1.6 /$ & $3.1 /$ \\
\hline $\mathrm{SMX} / \mathrm{TMP}$ & 0.021 & 0.041 & 0.017 & 0.021 & 0.027 & 0.034 & 0.082 & 0.082 & 0.16 \\
\hline
\end{tabular}

${ }^{a}$ The $S$. aureus COL strain was used to represent the T51M E208K combination since the isogenic mutant harboring this mutation set could not be generated.

${ }^{\mathrm{b}} \mathrm{SMX} / \mathrm{TMP}$ combination was prepared at a 19:1 combination. 
combination MIC exceeded the combined MICs of the primary and secondary mutations alone for nearly all sulfonamides. The COL strain representing the T51M E208K set had the highest MIC values overall, but genetic variations in this strain could have additional effects on susceptibility (Table 2-5).

\section{Altered susceptibility of isogenic mutants to TMP and co-trimoxazole}

To explore the potential for effects of DHPS mutation-induced collateral sensitivity against DHFR-targeted agents, the TMP susceptibility among the isogenic mutants was determined both alone and in combination with the common partner drug SMX. DHFR is two steps downstream of DHPS in the FBP and targeting both enzymes with the co-trimoxazole combination results in synergy (Figure 1-2).

Interestingly, the primary mutations imparted a remarkable decrease in the MIC for TMP. Secondary mutations alone had negligible changes to the TMP MIC, but the MICs among the double mutants showed a partial restoration of TMP susceptibility closer to WT levels of $1.6 \mu \mathrm{g} / \mathrm{mL}$ (Table 2-5 and Figure 2-3). The addition of $p$ ABA to the testing media universally restored the MIC for all affected strains (Figure 2-3). This provides interesting implications as to how closely the enzymes of the FBP are interconnected, which could explain the synergy observed within the co-trimoxazole combination. Primary DHPS mutations were shown to impart a fitness cost in terms of biochemical function (Table 2-4), which likely renders the downstream enzyme DHFR more crucial to overall cell viability, resulting in a collateral sensitivity effect towards TMP. Secondary mutations appear to not only increase sulfonamide resistance, but also rescue defects in both biochemical fitness (Table 2-4) as well as restore the TMP and cotrimoxazole MIC (Table 2-5). Addition of $p$ ABA may boost the catalytic output of DHPS and outcompete sulfonamides, reducing the downstream consequences of primary mutation on DHFR. These effects highlight the interplay between the two enzymes and suggests the synergy and efficacy of the co-trimoxazole combination can be attributed to collateral sensitivity effects occurring within antifolate combination therapy.

Although co-trimoxazole is formulated at a SMX/TMP ratio of 5:1, in vitro testing typically occurs at a ratio of 19:1 to mimic the ratio of both drugs achieved in the serum after oral administration (Rein 1980). The mutant strains were tested against this combination and MICs were determined (Table 2-5). The single mutations did not show notable increases in resistance to the combination, but the increase in resistance among double mutants was at least three-fold. Although the primary mutations were more sulfonamide resistant than secondary mutations alone, the increased TMP susceptibility maintained the co-trimoxazole MIC closer to WT levels, with only a two-fold increase seen for F17L which showed the strongest overall resistance to the sulfonamide panel as a single mutant. When a primary mutation is present, a secondary mutation may be necessary to compensate for the heightened TMP susceptibility and impart high-level resistance to the SMX/TMP combination. Therefore, in addition to restoring biochemical fitness (Table 2-4), secondary mutations rescue the collateral sensitivity imparted by DHPS primary mutations towards TMP (Figure 2-3). 


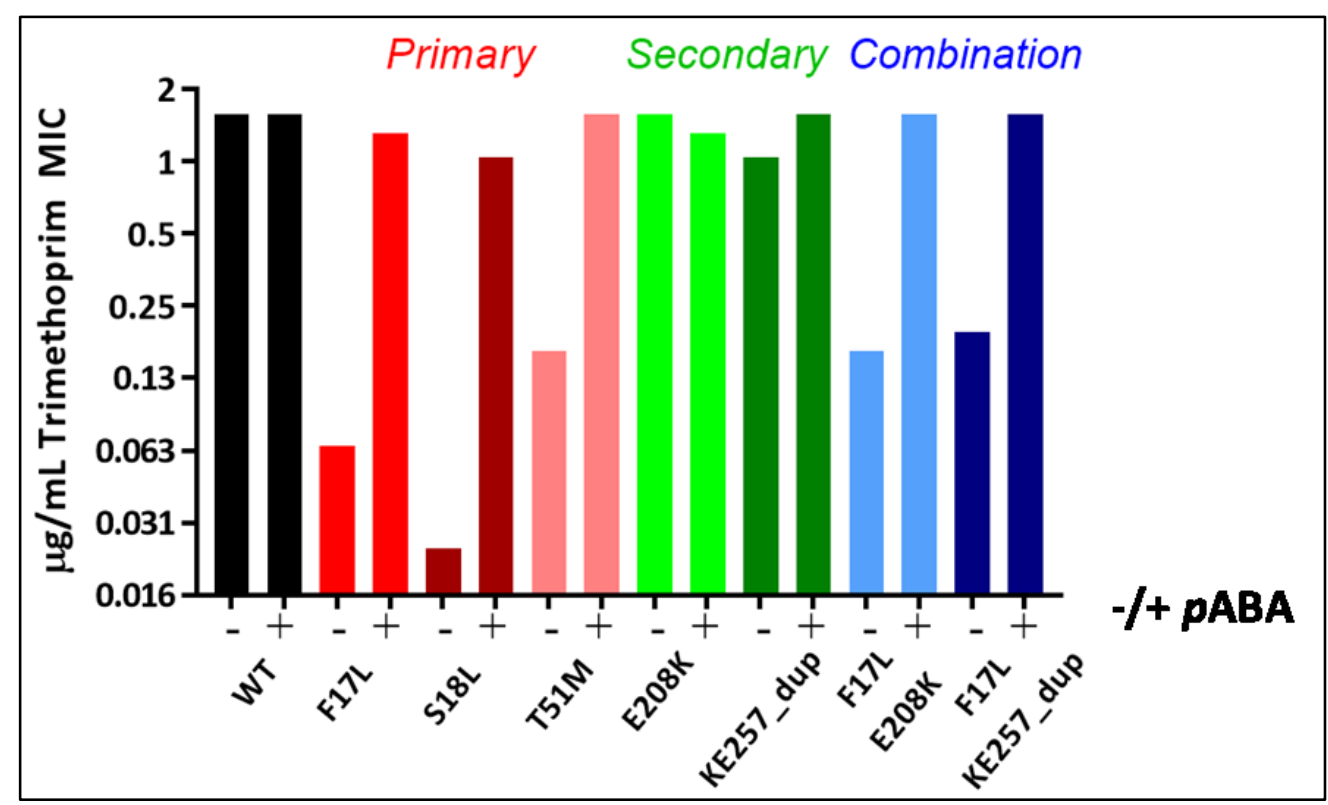

Figure 2-3. Trimethoprim susceptibility profile of isogenic DHPS mutant panel. This data represents the TMP MIC data in Table 2-5 and includes additional MIC values determined in the presence of $p \mathrm{ABA}$ supplementation. The presence or absence of 5 $\mu \mathrm{g} / \mathrm{mL} p \mathrm{ABA}$ is represented by $\mathrm{a}+$ or - sign, respectively. 


\section{Growth curve studies}

To determine whether the DHPS mutations directly associated with resistance resulted in reduced fitness, growth rates were determined for each mutant either as single mutations or in their observed clinical combination. It was found that only the S18L and T51M primary mutations had a significant impact on doubling time (Figure 2-4). This indicates that mutations in the flexible loop region where both native substrate $p \mathrm{ABA}$ and sulfonamides bind results in the highest fitness cost. However, F17L, which resides in the flexible loop region, did not show a fitness cost in this model. The higher-level resistance of F17L in comparison to the other primary mutations and the lack of an associated fitness cost may explain its frequency in $S$. aureus and other organisms in comparison to the other two primary mutations (Tables 2-2 and 2-3).

\section{Galleria mellonella infection model}

The larvae of the greater wax moth, Galleria mellonella, can serve as cheap and effective host organisms for in vivo rescue experiments (Ramarao 2012, Tsai 2016). This model was used to study both the virulence and resistance of the isogenic mutants from an in vivo standpoint. The wax moth larvae infection model was first optimized to determine the inoculum density necessary to achieve a median lethal dose (LD50) with the WT strain at 72 hours post-infection. This three-day time span allows the opportunity for several live/dead counts before the larvae begin the pupation stage (Tsai 2016). Using the USA300 AH1263 WT strain to optimize this model, it was found that approximately $10^{5}$ colony-forming units (CFU) of bacteria per injection could achieve approximately 50\% mortality at 72 hours (Figure $2-5 \mathbf{A}$ ).

To test for resistance in this model, it is necessary to be able to rescue the larvae hosts from WT infection using a sulfonamide. It was found that SMX is soluble in a carrier solvent of PBS and 5\% DMSO up to $100 \mathrm{mg} / \mathrm{mL}$. Daily administration of 100 $\mathrm{mg} / \mathrm{kg}$ SMX resulted in a significant rescue of the larvae from WT infection (Figure 25B). A control group receiving an equivalent number of injections per day with the PBS/DMSO carrier showed minimal death over the 72-hour span.

The entire isogenic mutant panel was subjected to this infection model to monitor for any significant reduction in virulence. No significant difference was observed according to the Mantel-Cox (Log-Rank) analysis of mortality-based virulence among all of the mutants relative to the WT strain (Figure 2-6). However, overall mortality rates at the end of the study were lowest for S18L and T51M, which agrees with the reduced fitness represented by increased doubling times observed in the growth rate study (Figure 2-4). Interestingly, none of the mutants could be rescued by SMX treatment, indicating the resistance observed during MIC testing translates to in vivo resistance. This also confirms that the secondary mutations are not simply abrogating a fitness cost, but that they have direct roles in sulfonamide resistance. 


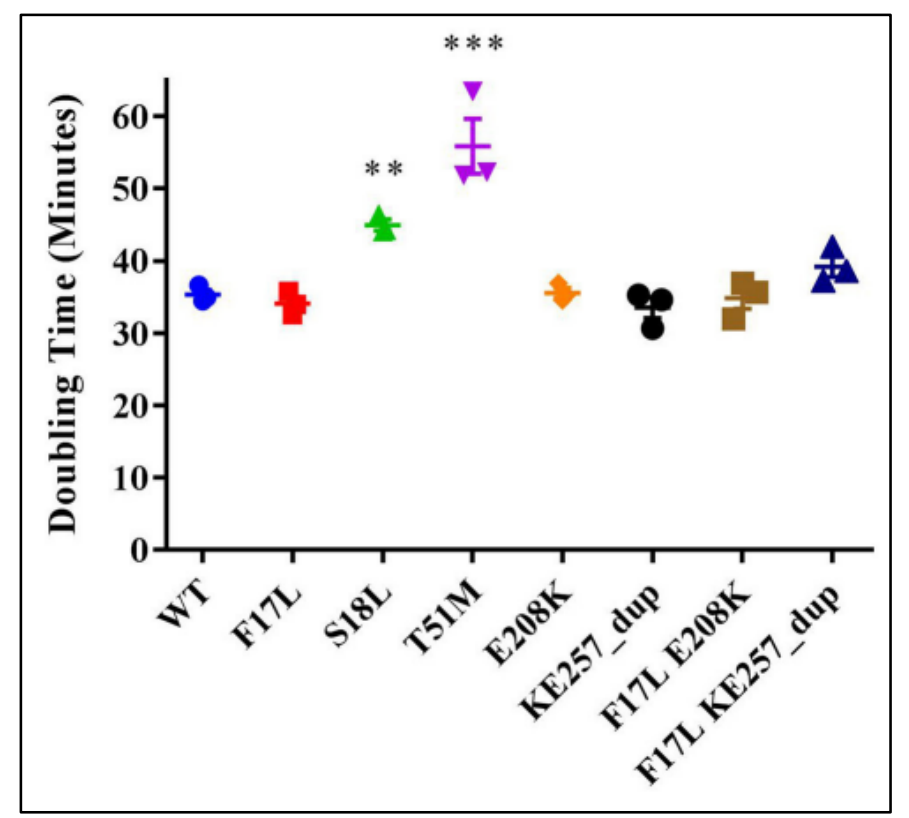

Figure 2-4. Doubling times of the isogenic DHPS mutant panel in S. aureus.

Doubling times were determined during the exponential growth phase of each strain. Significant differences in doubling times were determined by Dunnett's multiple comparisons. $* *$ indicates $\mathrm{p}<0.05, * * *$ indicates $\mathrm{p}<0.001$.

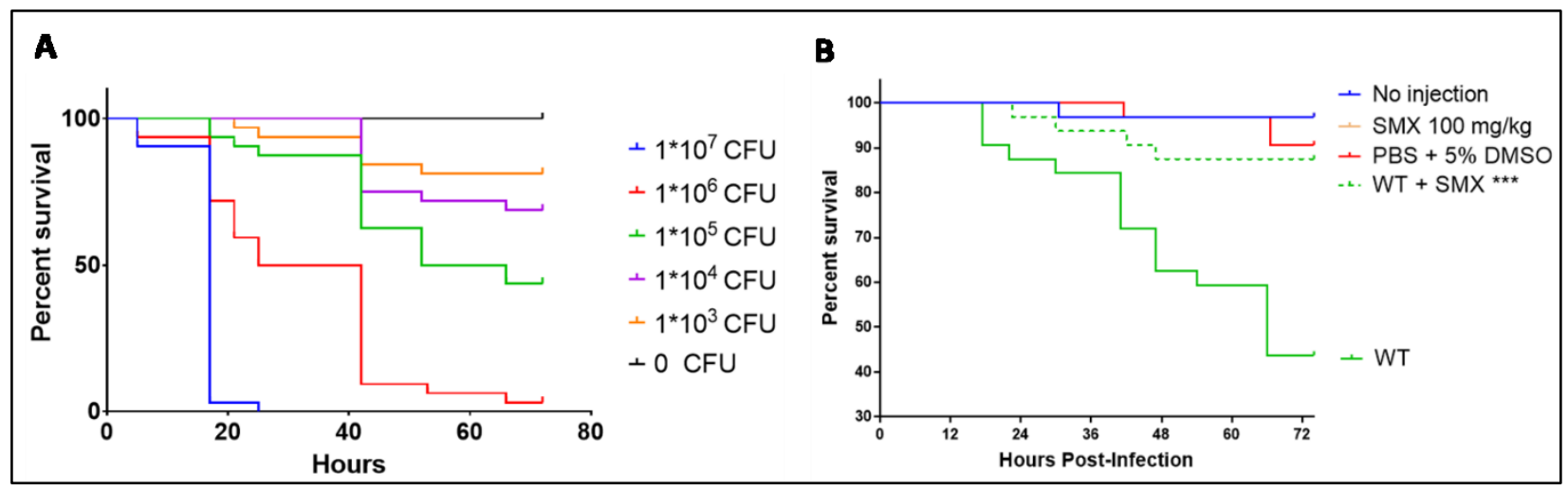

Figure 2-5. Optimization of wax moth larvae infection model.

A. Determination of USA300 inoculum density required to reach an LD50 at approximately 72 hours. B. SMX rescue of USA300 infection in G. mellonella infection model. *** indicates $\mathrm{P}<0.001$ using Mantel-Cox (Log-Rank) survival analysis. 


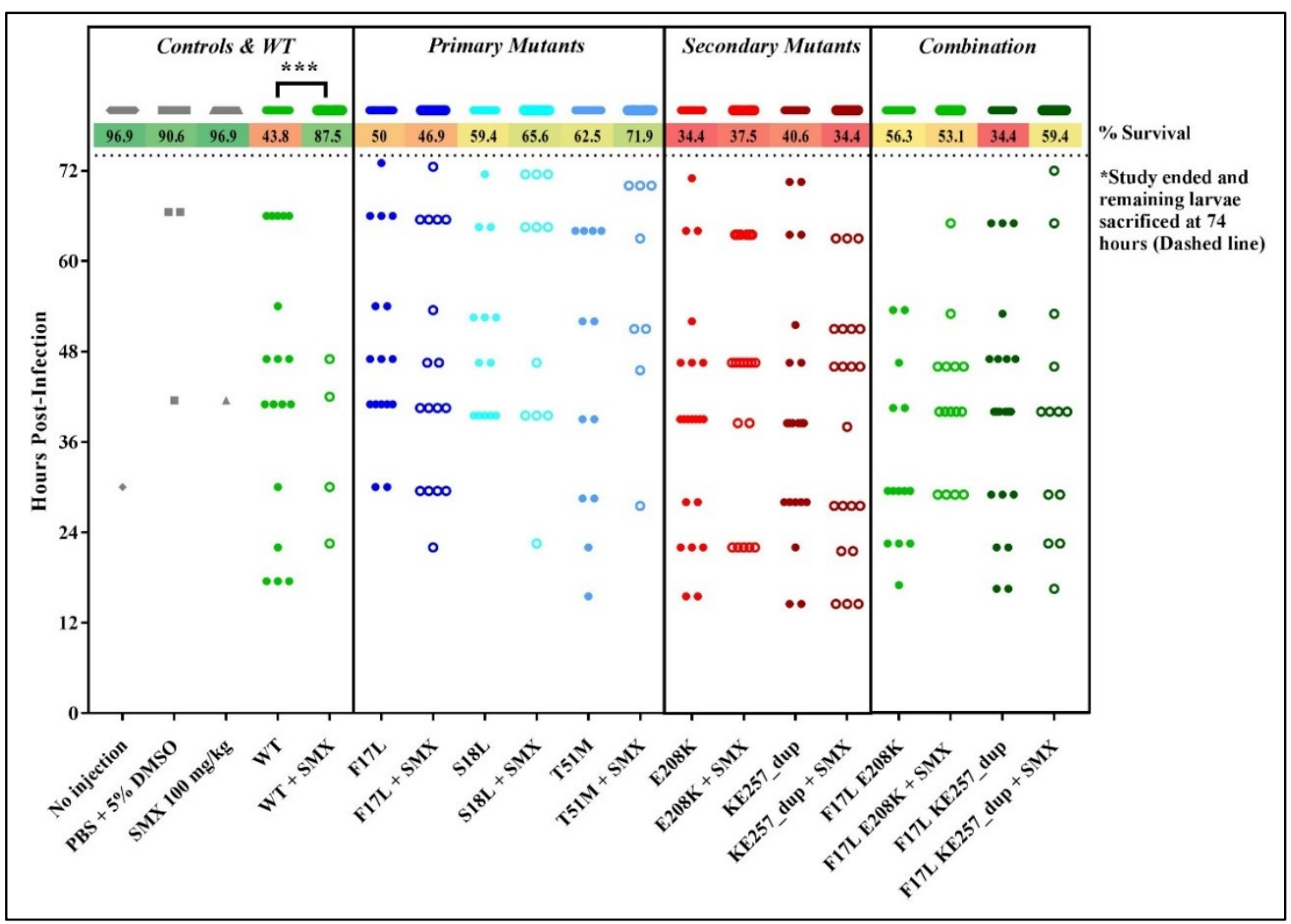

Figure 2-6. Galleria mellonella virulence and rescue study.

Mortality data are represented as a dot plot, with each dot representing an observed death. Closed dots are untreated larvae and open dots are SMX-treated larvae. ***indicates $\mathrm{P}<$ 0.001 via Mantel Cox (Log-Rank) survival analysis. 


\section{Structural characterization of the DHPS resistance mutations}

In order to study the DHPS resistance mutations from a structural standpoint, an X-ray crystallography study was initiated on all recombinant mutant proteins. The DHPS structure has a notable degree of disorder within the flexible loop system where both $p A B A$ and sulfonamides bind. This has caused the structural study of the active site region of DHPS from $S$. aureus and other species to be especially difficult (Hampele 1997). Crystallization and data collection were attempted on all variant enzymes, but only the F17L E208K structure was successfully obtained. This mutant structure was solved both in the apo state and in complex with 1530, which is a pterin-sulfisoxazole conjugate (Zhao 2016, Compound 16).

Although only the F17L E208K protein could be characterized through X-ray crystallography, the resulting structures provided valuable information on resistance mechanisms imposed by both mutations. Previously reported structures of $Y$. pestis DHPS in the transition state were valuable in the study and modeling of the active site locale and adjacent salt bridge array affected by secondary mutation (Yun 2012). Fortunately, several of the active site residues are well conserved between the two species. Modeling of the SaDHPS transition state using YpDHPS 3TYZ predicted several of the loop 1-2 residues known to coordinate binding of $p$ ABA and SMX likely maintained the same roles in S. aureus. S18 does not appear to have direct interactions with $p$ ABA but may have stabilizing effects on loop 1 . T51 interacts with the $p$ ABA amino group through hydrogen bond formation and stabilizes the amino group of the $\mathrm{C} 11$ carbon of DHP to assist in bond formation with $p$ ABA (Figure 2-7A).

The crystal structures of both apo- and 1530-bound SaDHPS with the F17L E208K double mutation were compared with the modeled SaDHPS transition state structure. The L17 residue in loop 1 of both structures assumes a similar position in the unbound structure to that of F17 in the modeled transition state, suggesting L17 may sterically interfere with the sulfonamide portion of 1530 (Figure 2-7A, B). The direct steric impact of the F17L mutation on sulfonamide binding was an expected effect given that it is located directly in the active site binding region of sulfonamides.

Compared to the primary mutations, the mechanisms of resistance imposed by the E208K secondary mutation are less immediately obvious. Structural studies, however, revealed an E208K-mediated mechanism of disfavoring inhibitor binding. E208 is part of a salt bridge array wherein E208 forms a salt bridge with R176, E179 forms a salt bridge with R204, and the two arginines at positions 176 and 204 interact by $\pi$-stacking. This array in the WT SaDHPS structure from 1AD1 is represented in Figure 2-7D. In the mutated structure, however, R204 is displaced and positioned so that it seems to sterically disfavor the position of the oxazole ring of 1530 which extends beyond the molecular space occupied by $p$ ABA. R176 forms a salt bridge with E179 instead of E208, suggesting disruption of the WT salt bridge array is an indirect mechanism of sterically disfavoring sulfonamide binding through mutation of E208.

These findings on the perturbation of the sulfonamide binding site induced by 


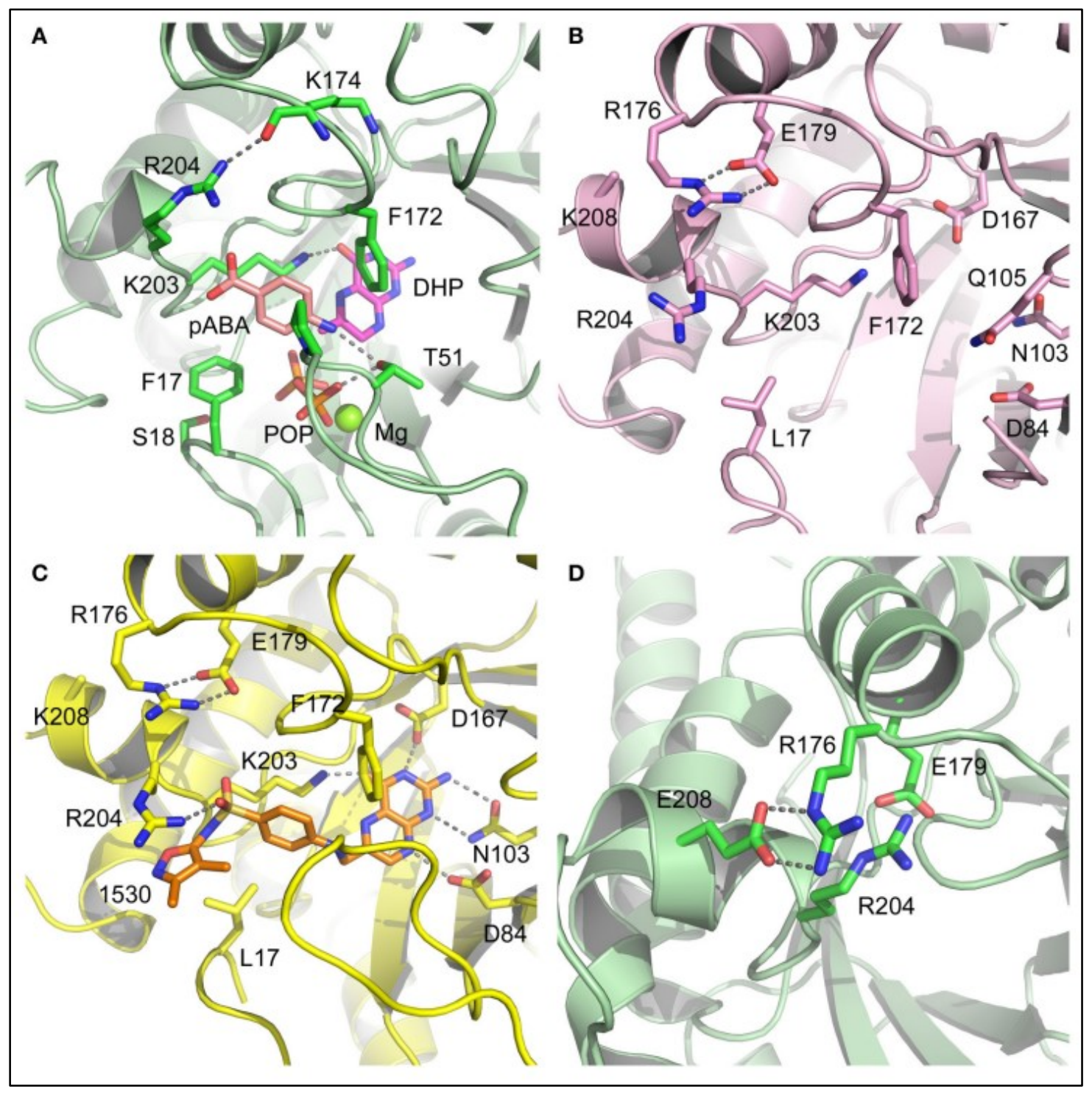

Figure 2-7. Structural characterization of the WT and F17L E208K salt bridge array.

A. SaDHPS transition state modeling based on the $Y$. pestis (PDB 3TYZ) structure. The DHPS protein is represented in green cartoon, highlighted residues are green carbon with stick representation, the ligands DHP and $p$ ABA are both in stick representation with magenta and salmon carbons, respectively, while PPi is orange. The magnesium cofactor is represented as a green ball. B. Crystal structure of the $S$. aureus F17L E208K salt bridge array. The protein in cartoon representation is shown in purple, and the residues are in stick representation with purple carbons. C. Crystal structure of F17L E208K salt bridge array in complex with 1530. The DHPS protein is in yellow cartoon, the residues are represented by yellow sticks, and 1530 is represented by orange sticks. D. WT SaDHPS salt bridge array proximal to the active site from the SaDHPS crystal structure 1AD1. The coloring and representation are the same as panel A. Salt-bridges and hydrogen bonds are represented by dashed gray lines for all panels. 
E208K emphasize the unexpected roles that secondary mutations can have on resistance. Pathogens can clearly develop sophisticated mechanisms through target alteration in regions distal from the site of drug binding. Before this work, sulfonamide resistance mutations were generally understood to exist directly in the active site loops 1 and 2, which directly interact with both sulfonamides and $p$ ABA, but resistance in $S$. aureus can apparently occur through additional secondary mutations that have indirect effects on sulfonamide binding site structure and additive effects on resistance (Table 2-5). Since the secondary mutation E208K occurs in a region of DHPS that is not well conserved in other species (Figure 2-2), this appears to be a resistance mechanism unique to $S$. aureus. These findings on the impact of mutations on regions extending beyond the binding sites of native substrates emphasize that next-generation inhibitors must remain within the substrate envelope to avoid impaction by mutations that readily arise against sulfonamides in clinical isolates. Studies on resistance mutations in hepatitis $\mathrm{C}$ virus also revealed that the highest level of resistance was also acquired by mutation against inhibitors extending beyond the molecular space within the substrate envelope (Romano 2010).

\section{Conclusions and Future Perspectives}

The basis for resistance caused by primary mutations within the loop 1-2 substructure are well characterized in this study and others, and the structural basis for resistance imparted by the E208K secondary mutation was explained for the first time in this body of work. Unfortunately, attempts to study the KE257_dup secondary mutation through X-ray crystallography were unsuccessful. An intriguing study characterizing the DHPS dimer interface for allosteric inhibitor development suggested interactions at this site influence breathing motions within the dimer that facilitate substrate release (Hammoudeh 2014). The dimer interface clearly imparts allosteric effects on the active site, but the exact basis for sulfonamide resistance achieved through alteration of this site has yet to be revealed.

The FBP remains promising for further drug discovery efforts due to the role of folates in numerous fundamental cell processes as well as the absence of many enzymes including DHPS from mammals (Bermingham 2000). As with all other major classes of antibiotics, the efficacy of our current arsenal of sulfonamides is threatened by continued observation of sulfonamide resistance globally. Effective next-generation DHPS inhibitors must be refractory to circulating mechanisms against established sulfonamides. In order to improve the current understanding of sulfonamide resistance mechanisms, a comprehensive characterization of DHPS mutations directly attributed to sulfonamide resistance was carried out using biological, structural, and biochemical approaches. The results presented within this chapter present important implications for future drug development towards DHPS. It was found that mutations in both the flexible loop region as well as in disparate regions of the enzyme influence the portion of sulfonamides that extends out of the substrate envelope (Figure 2-7). Therefore, next-generation DHPS inhibitors must avoid this molecular space that is most susceptible to circulating sulfonamide resistance mutations. 
Ongoing DHPS inhibitor development within our laboratory currently avoids probing the space beyond the $\mathrm{DHPP} / p \mathrm{ABA}$ substrate envelope. These inhibitors are expanding upon the pyridazine inhibitor series and are designed to remain within the conserved pterin binding site, wherein no resistance mutations are known to occur (Lever 1985, Zhao 2012). Beyond DHPS, there are also several enzymes of interest for inhibitor development within the FBP and related branches of bacterial metabolism. In the next chapter, further drug discovery efforts towards bacterial metabolism are pursued by employing a high throughput metabolic screen for new small molecule inhibitors disrupting metabolic processes related to folate biosynthesis. 


\section{CHAPTER 3. METABOLIC PROFILING OF ANTIFOLATES LEADS TO A SCREEN FOR NEW INHIBITORS OF BACTERIAL METABOLISM}

\section{Introduction}

\section{Metabolic networks associated with tetrahydrofolate are promising avenues for drug discovery}

The current antibiotic arsenal generally only targets a limited number of cell processes, including DNA synthesis, protein synthesis, and the cell wall (Haselbeck 2002, Brown 2013). New antimicrobials with unique MOAs would provide crucially needed treatment options when target-mediated resistance is presented against traditional antibiotic classes. A rich source of potential new antibiotic targets lies within bacterial metabolism, which is evolutionarily distinct from higher eukaryotes.

There is promising potential for antibiotic discovery towards both existing and new targets within the FBP (Sköld 2000, Zlitni 2013). Additionally, several allied pathways responsible for crucial metabolic processes are yet to be fully exploited for antibiotic discovery. Drug discovery campaigns towards bacterial metabolism have gained stamina in recent years using both structure-based and in vitro screening methodologies. Structure-based studies have allowed for the generation of improved inhibitors of 6-hydroxymethyl-7,8-dihydropterin pyrophosphokinase, which is immediately upstream of DHPS within the FBP (Dennis 2016). Propargyl-linked antifolates (PLAs) are showing the potential to successfully inhibit drug-resistant forms of DHFR, the final enzyme in the FBP, in MRSA strains (Reeve 2016). Recently, an inhibitor of $p \mathrm{ABA}$ biosynthesis was discovered in a differential metabolic screen in $E$. coli (Zlitni 2013). Beyond folate biosynthesis, the same screen identifying the $p$ ABA biosynthesis inhibitor also characterized inhibitors of biotin biosynthesis and glycine metabolism. Furthermore, an allosteric inhibitor of tryptophan synthase has recently been identified from a whole cell $M$. tuberculosis screen against a diversity-oriented synthetic library (Wellington 2017).

\section{Obstacles of antimetabolite drug discovery}

A common issue with early stage development of antimetabolites is the fact that nutrients within common testing media can interfere with the ability of certain antimicrobials to inhibit bacterial growth. The MICs of antimetabolites representing both experimental and existing antibiotic compounds are known to be masked by nutrient-rich testing medias (Zlitni 2013). When the product of a target enzyme is available to the cell in sufficient quantities, i.e. nutrients within the media, inhibiting the function of the enzyme may not result in growth inhibition as there is no starvation effect. Furthermore, bacterial metabolism is extensively regulated, and the transcription of the biosynthetic machinery is often dependent on closely associated metabolites (Baba 2006). In a 
nutrient-rich environment, the intended antimicrobial target may not be expressed in sufficient quantities for the intended growth inhibitory effect. Conversely, in the absence of specific metabolites, the transcription of de novo biosynthetic machinery is often greatly upregulated and necessary for survival. This drastic change in gene transcription has been observed in E. coli, wherein 119 genes are known to be essential in a minimal nutrient environment, but dispensable in rich growth media (Baba 2006).

Mueller Hinton (MH) and lysogeny broths (LB) are very common bacterial testing media, but they are rich in peptides and vitamins that represent both intermediates and downstream products of folate biosynthesis. Isosensitest broth (ISB) is often used for antifolate susceptibility testing due to significantly reduced concentrations of thymidine and $p \mathrm{ABA}$, but this media is still rich in peptides. Various amino acids are downstream products of folate biosynthesis and methionine is known to mask antifolate MICs (Zlitni 2013). Molecules in early-stage development often have MICs that are close to the upper limit of detection, and even a subtle decrease in potency can cause the MIC to be undetectable. This inability to detect a MIC dramatically hinders the ability to improve biological activity through a structure-activity relationship (SAR) approach.

A preferred testing media for early-stage development of antifolates and other antimetabolites would completely lack all amino acids and as many vitamins as possible. The high-throughput screen (HTS) carried out by Zlitni and colleagues, which identified the inhibitors of $p$ ABA biosynthesis, biotin biosynthesis, and glycine metabolism in $E$. coli, was carried out against whole cell E. coli in the M9-based minimal media ATCC 2511 (Zlitni 2013). Initial indications of the MOA of the primary hits was evaluated with "rescue studies" carried out by adding individual metabolites to the M9 media to test for antagonism of the growth inhibitory action of these molecules. This screen yielded several interesting new antimetabolite compounds, including MAC173979, which is an inhibitor of $p \mathrm{ABA}$ biosynthesis and is very closely linked to folate biosynthesis (Zlitni 2013). The potency of this hit compound could be antagonized by supplementation with both $p \mathrm{ABA}$ and L-methionine, which is a similar metabolic profile to that of conventional sulfonamide drugs.

Within this chapter, whole cell testing conditions are optimized to detect the growth inhibition of next-generation antifolates against the GN pathogen $E$. coli without the interference of metabolites related to folate biosynthesis. The inclusion of two additional testing conditions in the presence of the DHPS substrate $p$ ABA and the folate derivative methionine result in detectable MIC shifts indicating an interaction with the target pathway. This approach augmented an SAR study of a novel series of pterinsulfonamide conjugates designed to simultaneously engage two distinct binding sites on the DHPS enzyme.

These conditional antimetabolite testing methods were adapted to a HTS for small molecule inhibitors of E. coli. A Bioactives collection of known therapeutics including antibiotic antifolates confirmed this screening approach, after which approximately 13,000 compounds were screened. After excluding known antibiotics, a collection of validated hits with varying sensitivity to the amino acid methionine were obtained. These 
hits consisted of both existing therapies characterized for human targets as well as a variety of small molecules yet to be characterized for biological activity. These efforts have yielded tools for better detection and improvement of biological activity of novel antifolates. Additionally, the HTS resulted in a collection of new GN-effective inhibitors that could potentially have an exciting new antimetabolite MOA in a time when new first-in-class inhibitors are desperately needed.

\section{Materials and Methods}

\section{Differential susceptibility testing in M9 minimal media}

MIC broth dilution testing was performed as previously described in Chapter 2 . E. coli K12 MG1655 was purchased directly from ATCC and only cultured in M9 for initial and subsequent stock generations to avoid auxotrophy. MIC determination of pterin-sulfa conjugates, primary screening, and secondary validation experiments were all carried out in M9 using this strain. M9 media was prepared as described for ATCC media 2511 . For every liter of media, $200 \mathrm{~mL}$ of $5 \mathrm{X} \mathrm{M} 9$ salt solution, $20 \mathrm{~mL}$ of $20 \% \mathrm{w} / \mathrm{v}$ glucose, $2 \mathrm{~mL}$ of $1 \mathrm{M} \mathrm{MgSO}_{4}, 0.1 \mathrm{~mL} \mathrm{CaCl} 2$, and $0.1 \mathrm{~mL}$ of a $0.5 \% \mathrm{w} / \mathrm{v}$ solution of thiamine were combined and brought to the final volume and filter-sterilized. When needed, supplements were added at concentrations of $5 \mu \mathrm{g} / \mathrm{mL}$ for $p \mathrm{ABA}$ or $20 \mu \mathrm{g} / \mathrm{mL}$ for L-methionine.

\section{High throughput screen in nutrient-restricted media}

Thirteen microliters of M9 media was dispensed into each well of a Nunc 384well optical bottom plate using the Thermo Scientific Wellmate Matrix Dispenser. A PerkinElmer BackSeal was applied to the bottom of the plate to accommodate luminescence reads. Source plates containing approximately $10 \mathrm{mM}$ compound were used to stamp $121 \mathrm{~nL}$ into the assay plate using a Biomek FXP Automated Liquid Handler. Columns 1, 2, 13, and 14 were reserved for controls, into which CAM or SMX were added from a control plate. A mid-log culture of $E$. coli K12 MG1655 was diluted to an OD600 of 0.001 in ATCC medium 2511 before dispensing $13 \mu \mathrm{L}$ into each well of the drugged assay plates using the Thermo Scientific Wellmate Matrix Dispenser, resulting in $1.3 \times 10^{4} \mathrm{CFU}$ per well. Concentrations varied slightly in the library source plates, but the final assay concentration for each compound was approximately $50 \mu \mathrm{M}$.

After 16 hours of incubation at $37^{\circ} \mathrm{C}, 26 \mu \mathrm{L}$ freshly prepared BacTiter-Glo ${ }^{\mathrm{TM}}$ was added to each well. The plates were allowed to shake for four minutes followed by a luminescence read within thirty minutes of adding the reagent. Four columns total were committed to controls, with two of these columns containing two-fold serial dilutions of both CAM and SMX. Therefore, MICs of these standard drugs could be determined within each plate, and the MIC of SMX was shifted by the addition of pABA and methionine. The other two columns containing alternating wells with either $50 \mu \mathrm{M} \mathrm{CAM}$ 
or DMSO, representing full inhibition or full growth, and were used to calculate the Zfactor of each plate. The high-throughput data was analyzed using the Robust Interpretation of Screening Experiments (RISE) application, which was written with Pipeline Pilot (Accelrys, v. 8.5) using the R program (R Core Team 2015).

\section{Secondary validation of primary hits and testing for rescue effect}

Hits from the primary screen that showed $\geq 50 \%$ growth inhibition were validated using a traditional MIC assay. Three replicates of the MIC were performed on three separate days. The resulting MIC values were averaged, and hits that maintained an $\mathrm{MIC}_{80}$ of $50 \mu \mathrm{M}$ or less were considered validated hits.

\section{Results and Discussion}

\section{Minimal media conditions enable SAR study of pterin-sulfa conjugates}

Antifolate compounds developed in our laboratory have displayed high affinity engagement of DHPS and structural studies have shown that they interact with the enzyme in areas that are less prone to common sulfonamide resistance mechanisms (Zhao 2012, Hammoudeh 2014). Despite these advantages, the measurement of biological activity under standard testing conditions is an obstacle towards the further development of these compounds. Similar to conventional antifolates, it is likely that nutrients present in standard testing media interfere with the detection of growth inhibition.

A new series of pterin-sulfa compounds were developed to simultaneously engage the DHPS enzyme in the conserved DHPP binding site and the traditional sulfonamide binding site (Zhao 2016). Eight molecules were synthesized with a fixed pterin moiety conjugated to each of eight different sulfonamides (Table 3-1). Instead of the rich MIC testing broths LB, MH, and ISB, a M9-based minimal media broth (ATCC 2511 or M9) was used to test for MIC activity.

The pterin-sulfa compounds have no detectable MIC against E. coli in either MHII or ISB (data not shown). When these compounds were tested in the M9-based media ATCC 2511 (M9), however, MICs against E. coli K12 were observed to be as low as $10.9 \mu \mathrm{M}$ (Table 3-1). Similar to the antifolates tested in the Zlitni et al. study, the action of these conjugates could be strongly antagonized by $p$ ABA and to a lesser extent Lmethionine (Zlitni 2013, Zhao 2016). The corresponding sulfonamides were also tested and displayed a similar pattern of antagonism through supplementation (Table 3-1). Furthermore, the potency of the pterin-sulfa conjugates had a high degree of correlation with the potency of the associated sulfonamide. 
Table 3-1. The MICs of pterin-sulfa conjugates can be rescued by metabolites related to folate biosynthesis.

\begin{tabular}{|c|c|c|c|c|}
\hline \multirow{2}{*}{ Compound \# } & \multirow{2}{*}{$\mathbf{R}$} & \multicolumn{3}{|c|}{ Minimal inhibitory concentration $E$. coli $\mathrm{K} 12$, in $\mu \mathrm{M}^{\mathrm{a}}$} \\
\hline & & M9 media & M9 plus $5 \mu \mathrm{g} / \mathrm{mL} p \mathrm{ABA}$ & M9 plus $20 \mu \mathrm{g} / \mathrm{mL}$ methionine \\
\hline 15 & & $10.9(0.8)^{\mathrm{b}}$ & $467(263)$ & $29.0(1.5)$ \\
\hline 16 & & $28.3(1.5)$ & $>412(249)$ & $47.0(5.9)$ \\
\hline 17 & & $17.1(1.6)$ & $>470(666)$ & $78.0(6.2)$ \\
\hline 18 & & $29.5(2.7)$ & $>471(669)$ & $98.0(6.3)$ \\
\hline 19 & & $17.2(1.6)$ & $>412(537)$ & $86.0(3.4)$ \\
\hline 20 & & $34.3(3.8)$ & $>412(>644)$ & $172(10.1)$ \\
\hline 21 & & $197(25)$ & $236(>934)$ & $236(50)$ \\
\hline 22 & & $136(22)$ & $>514(>934)$ & $>200(58)$ \\
\hline Ampicillin & & 1.6 & 2.0 & 2.1 \\
\hline Chloramphenicol & & 8.5 & 9.7 & 9.7 \\
\hline Trimethoprim & & 1.7 & 1.4 & 0.7 \\
\hline
\end{tabular}




\section{Table 3-1. Continued.}

Modified with permission from Zhao Y, Shadrick WR, Wallace MJ, Wu Y, Griffith EC, Qi J, Yun MK, White SW, Lee RE. Pterinsulfa conjugates as dihydropteroate synthase inhibitors and antibacterial agents. Bioorg Med Chem Lett. 2016 Aug 15;26(16):3950-4. ${ }^{a}$ Average of 3 experiments.

${ }^{\mathrm{b}}$ Corresponding values for parent sulfonamides shown in parentheses. 


\section{Assay validation for the conditional metabolic screen through a preliminary screen of known Bioactive compounds}

The revealed activity of the pterin-sulfa series against the GN pathogen $E$. coli K12 enabled the potential for pursuing a HTS for new antimetabolites that could inhibit the growth of this clinically significant pathogen. The primary screen would be conducted in the minimal media environment to avoid interference of MIC activity by metabolites present in the testing media. Two additional conditions, the presence of $p$ ABA or L-methionine, could be included as an indication of interaction with the biosynthesis of folate and closely related pathways (Zlitni 2013).

To investigate the feasibility of this screening approach, a library of Bioactive molecules was initially tested. This library consists of 1,831 molecules with known biological activity and therapeutic utility against a wide range of maladies such as cancer, cardiomyopathies, and infections. A range of antibiotic classes including sulfonamide drugs and other antifolates are also present in this library, allowing for several internal controls for both $E$. coli growth inhibition and the conditional metabolic screen. The initial screen was set up in 384 well plates with designated wells for the positive control, CAM, and the negative control, DMSO, representing 100\% growth inhibition and no growth inhibition, respectively. An internal assay control represented by a SMX doseresponse was also included to show that antagonism by $p$ ABA and L-methionine could be achieved. Each drug from the Bioactives collection was added to the assay plates at a final concentration of approximately $50 \mu \mathrm{M}$ in $\mathrm{M} 9$, and the plates were inoculated and incubated similar to a standard MIC (see methods). Growth inhibition was determined based on a luciferase-based readout that is sensitive to the amount of ATP in each well, corresponding to the number of viable cells.

The Robust Interpretation of Screening Experiments (RISE) application was utilized for analysis of the screening data (R Core Team, 2015). Z-factors ranging between 0.43 and 0.69 were achieved within the Bioactives screen, with an average Zfactor of 0.59 (Figure 3-1), indicative of an excellent assay (Zhang 1999). Only four plates were slightly below this threshold.

In addition to the Z-factor calculations for the control compounds, the data for all molecules within the Bioactives screen were again analyzed using RISE. Out of the 1,831 compounds, 78 exhibited at least $50 \%$ growth inhibition relative to the internal controls, resulting in a hit rate of $4.3 \%$. Most of these hits were known antimicrobial compounds. When these antimicrobial compounds were categorized by MOA, it was apparent that antifolates indeed showed a unique metabolic profile, with the action of nearly all antifolates, with the exception of TMP, being completely antagonized by $p \mathrm{ABA}$. Methionine rescued the growth inhibition of antifolates to a lesser extent, and also exhibited an effect on some inhibitors of DNA and protein synthesis (Figure 3-2). 


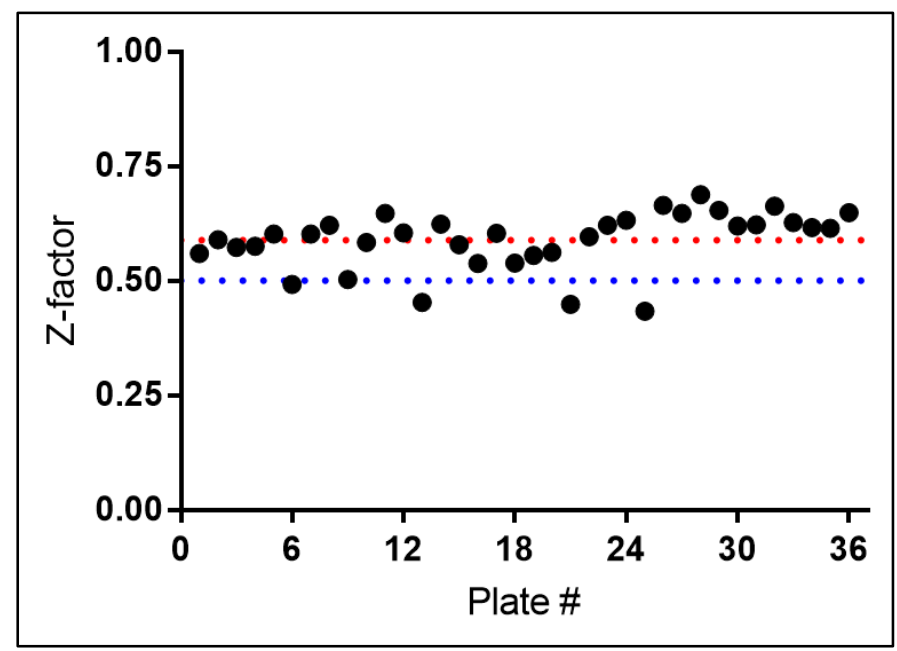

Figure 3-1. $\quad Z$ factors observed for the preliminary Bioactives screen.

$Z$-factors are determined based on internal positive and negative controls in each assay plate. Blue dashed line at 0.5 represents the threshold for an excellent assay. Red dashed line at 0.59 represents the average Z-factor of the Bioactives screen.

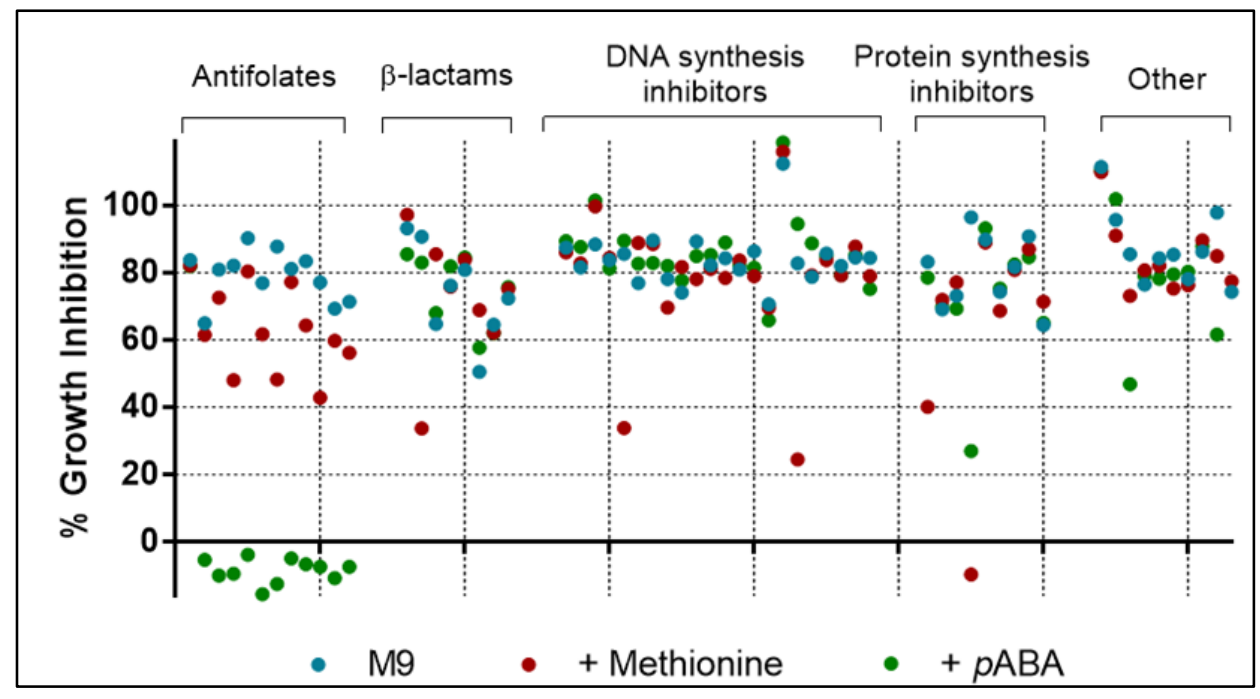

Figure 3-2. Growth inhibition and antagonism by key metabolites observed by known antimicrobials in the Bioactives collection.

Blue dots represent percentage growth inhibition in M9 media. Red and green dots represent percentage growth inhibition in the presence of either $20 \mu \mathrm{g} / \mathrm{mL}$ methionine or $5 \mu \mathrm{g} / \mathrm{mL} p \mathrm{ABA}$, respectively. 


\section{High-throughput screen of nearly 13,000 compounds}

Since the quality of the HTS methods and antimetabolite detection strategy was successfully validated using the Bioactives collection, the HTS was further expanded to three additional libraries including a set of "Lead-Like" molecules, a Small Fragment library, and an in-house library of antifolates. This led to a total of 12,597 molecules that underwent the HTS. The Lead Like set consists of 10,041 molecules with diverse chemical scaffolds that are suitable for lead optimization with a molecular weight at or below 400. The small fragment library is comprised of 750 compounds, wherein $98 \%$ of the molecules in this set have a molecular weight below 200. Finally, a collection of antifolate compounds generated in-house was assembled. This consisted of 335 compounds in total, with ten of them being established sulfonamides.

Due to the large number of compounds included in the screen, these three additional libraries were first screened only in M9. The growth inhibition threshold for hit determination was again set at 50\%, and 136 primary hits were obtained, resulting in a primary hit rate of $1.1 \%$ for the entire screen in M9 (Figure 3-3A). When the percentage growth inhibition of all experimental compounds and controls were plotted on a scatter plot, it was found that there was an adequate degree of separation between positive and negative controls (Figure 3-3B). To further assess the quality of the primary screen, Zfactors were calculated for all M9 screening plates for all compounds tested. The average Z-factor was 0.62 , and all but one of the assay plates had a Z-factor above 0.5 (Zhang 1999) (Figure 3-4).

\section{Secondary validation of primary hits}

Among the primary hits showing $\geq 50 \%$ growth inhibition against E. coli, 54 molecules had no previously characterized antimicrobial activity and were selected for secondary analysis. These molecules underwent traditional MIC testing in unsupplemented M9, M9 with $20 \mu \mathrm{g} / \mathrm{mL}$ methionine, and M9 with $5 \mu \mathrm{g} / \mathrm{mL} p$ ABA. Nineteen compounds had an $\mathrm{MIC}_{80}$ of $\leq 50 \mu \mathrm{M}$ and were considered validated hits (Table 3-2).

Most of the validated hits originated from the Bioactives or Lead-Like libraries, and one compound originated from the Small Fragment Library. From the Bioactives collection, the validated hits were mostly oncological agents disrupting metabolic processes related to DNA. Notably, five inhibitors of thymidylate synthase (TS) were confirmed secondary hits (Table 3-2). These drugs are all analogs of fluorouracil. Although not situated directly in the classic FBP, TS is only two enzymatic steps downstream of THF (Figure 3-5). TS converts deoxyuridine monophosphate (dUMP) to deoxythymidine (dTMP), which is one of the four nucleotide bases in DNA. N5,N10methylenetetrahydrofolate acts as a methylene and hydride donor during this reductive methylation reaction. Along with dTMP, 7,8-dihydrofolate is also produced during this reaction and recycled back to DHFR at the last step of the FBP. 


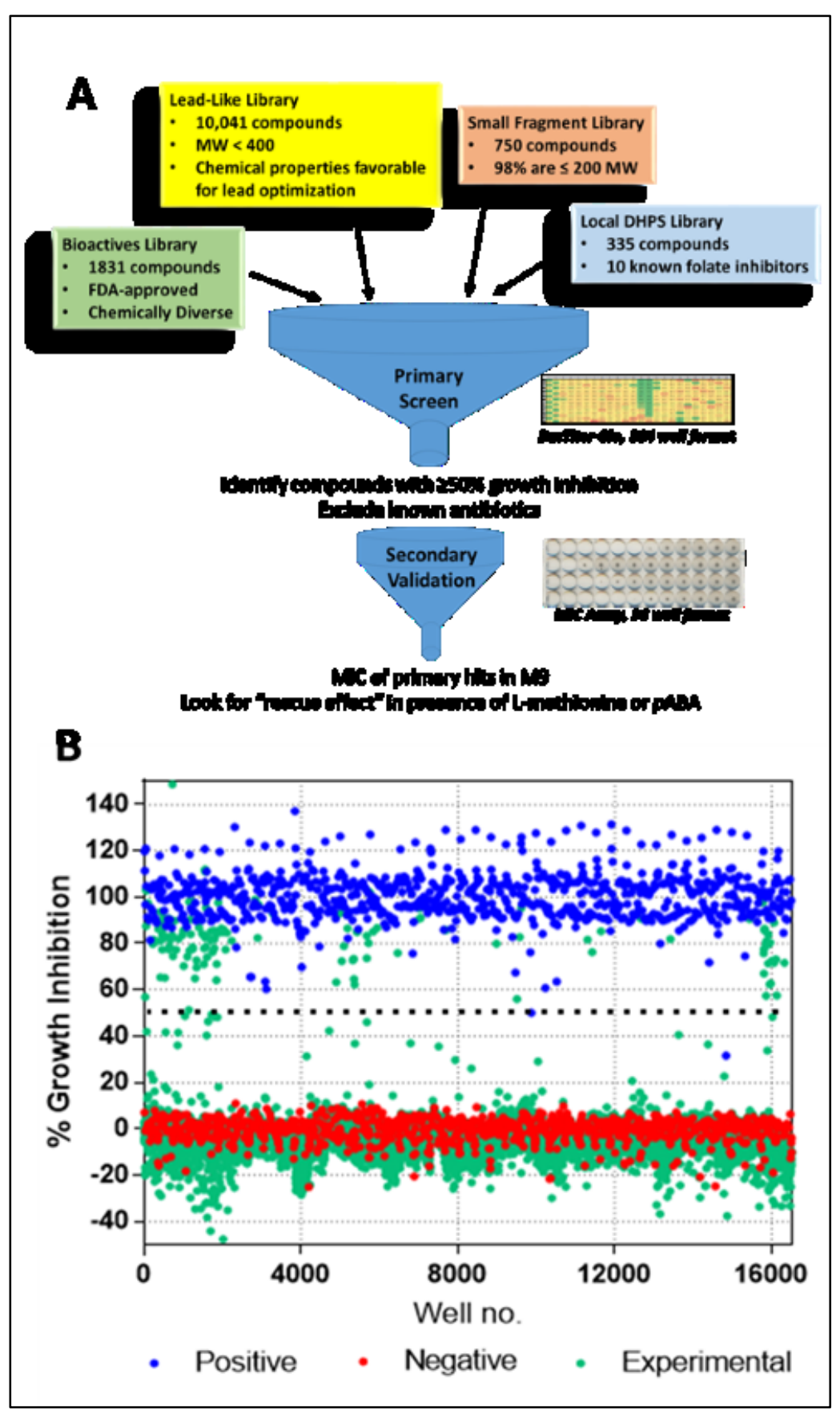

Figure 3-3. HTS of nearly 13,000 compounds in nutrient-restricted broth. A. Schematic of the overall screen. B. Scatter plot of percentage E. coli growth inhibition in M9 for all compounds tested. Dashed line at 50\% growth inhibition indicates the minimum percentage of growth inhibition required for hit determination. 


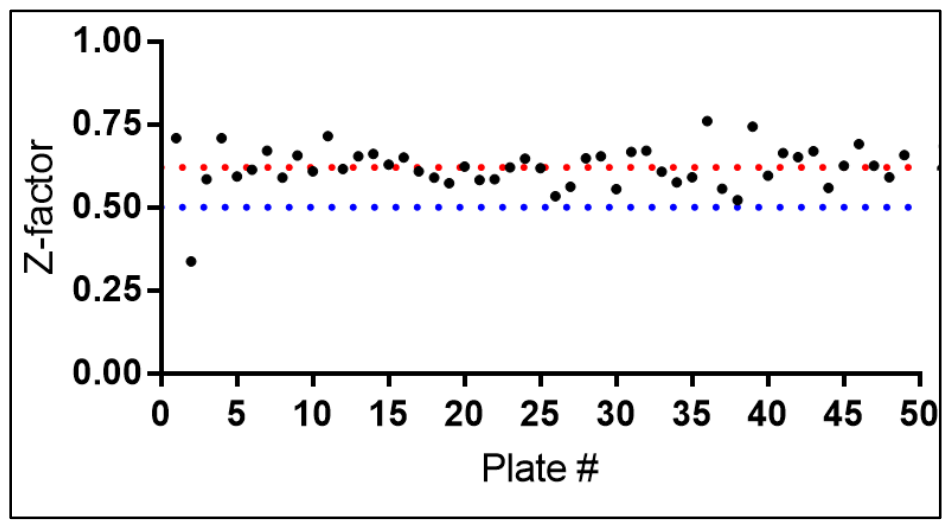

Figure 3-4. $\quad Z$ factors of the entire screen, $M 9$ only.

Blue dashed line at 0.5 represents the threshold for an excellent assay. Red dashed line at 0.62 represents the average Z-factor for the complete primary screen in M9. 
Table 3-2. Validated hits from the conditional metabolic screen.

\begin{tabular}{|c|c|c|c|c|c|}
\hline \multirow{2}{*}{$\begin{array}{l}\text { Common } \\
\text { Name/SJ\# }\end{array}$} & \multirow{2}{*}{$\begin{array}{c}\mu \mathrm{M} \\
\mathrm{MIC}_{80}{ }^{\mathrm{a}}\end{array}$} & \multicolumn{2}{|c|}{ Fold Shift MIC } & \multirow{2}{*}{$\begin{array}{l}\text { Screening } \\
\text { Library }\end{array}$} & \multirow{2}{*}{ Mechanism of Action } \\
\hline & & Methionine $^{\mathrm{c}}$ & $p \mathbf{A B A}^{\mathrm{d}}$ & & \\
\hline 5-fluorouracil & 0.16 & - & - & Bioactives & $\begin{array}{l}\text { Thymidylate synthase } \\
\text { inhibitor }\end{array}$ \\
\hline Carmofur & 0.20 & - & 0.75 & Bioactives & $\begin{array}{l}\text { Thymidylate synthase and } \\
\text { acid ceramidase inhibitor }\end{array}$ \\
\hline Floxuridine & 0.21 & 1.3 & - & Bioactives & $\begin{array}{l}\text { Thymidylate synthase } \\
\text { inhibitor }\end{array}$ \\
\hline Decitabine & 0.32 & 1.6 & 0.83 & Bioactives & $\begin{array}{l}\text { DNA methyltransferase } \\
\text { inhibitor }\end{array}$ \\
\hline Azacytidine & 1.5 & 0.71 & 0.71 & Lead-Like & $\begin{array}{l}\text { Cytidine analog, treats } \\
\text { myelodysplastic syndrome }\end{array}$ \\
\hline Zebularine & 1.8 & - & 0.75 & Bioactives & $\begin{array}{l}\text { Cytidine analog, epigenetic } \\
\text { therapy }\end{array}$ \\
\hline Bleomycin & 3.1 & - & 0.67 & Bioactives & $\begin{array}{l}\text { Induces DNA strand } \\
\text { breaks }\end{array}$ \\
\hline STATTIC & 4.5 & 2.0 & - & Bioactives & STAT3 inhibitor \\
\hline Pifithrin & 6.5 & 2.0 & - & Bioactives & HSP70 inhibitor \\
\hline Cladribine & 8.9 & 1.7 & - & Bioactives & $\begin{array}{l}\text { Purine analog, inhibits } \\
\text { adenosine deaminase }\end{array}$ \\
\hline SJ000208968 & 12.6 & 4.0 & - & Lead-Like & Unknown \\
\hline Doxifluridine & 14.3 & - & 0.88 & Bioactives & $\begin{array}{l}\text { Thymidylate synthase } \\
\text { inhibitor }\end{array}$ \\
\hline $\begin{array}{l}\text { 5-fluoro-1- } \\
\text { (tetrahydro-2- } \\
\text { furfuryl)uracil }\end{array}$ & 18.1 & 1.3 & - & Bioactives & $\begin{array}{l}\text { Thymidylate synthase } \\
\text { inhibitor }\end{array}$ \\
\hline SJ000461885 & 20.9 & 4.8 & - & Lead-Like & Unknown \\
\hline SJ000209472 & 25 & 5.3 & - & Lead-Like & Unknown \\
\hline Cisplatin & 42 & 2.4 & - & Bioactives & $\begin{array}{l}\text { Inhibits DNA replication, } \\
\text { crosslinks purine bases }\end{array}$ \\
\hline SJ000866485 & 44 & - & - & $\begin{array}{l}\text { Small } \\
\text { Fragment }\end{array}$ & Unknown \\
\hline SJ000208489 & 50 & 5.3 & - & Lead-Like & Unknown \\
\hline SJ000129420 & 50 & 3.3 & 1.0 & Lead-Like & Unknown \\
\hline Chloramphenicol & 5.0 & 1.3 & - & NA & Control antibiotic \\
\hline Sulfamethoxazole & 0.39 & 2.0 & 849 & NA & Control antibiotic \\
\hline Trimethoprim & 0.69 & - & - & NA & Control antibiotic \\
\hline
\end{tabular}

${ }^{a} \mathrm{MIC}$ values and MIC shifts are an average of three replicate experiments.

${ }^{b}$ Fold shift MIC is the ratio of supplemented MIC over unsupplemented MIC. If ratio is equal to one, a dashed line is put in place of that data to represent an absence of an observable MIC shift.

${ }^{c} \mathrm{~L}$-methionine was supplemented at a final concentration of $20 \mu \mathrm{g} / \mathrm{mL}$.

${ }^{\mathrm{d}} p \mathrm{ABA}$ was supplemented at a final concentration of $5 \mu \mathrm{g} / \mathrm{mL}$. 


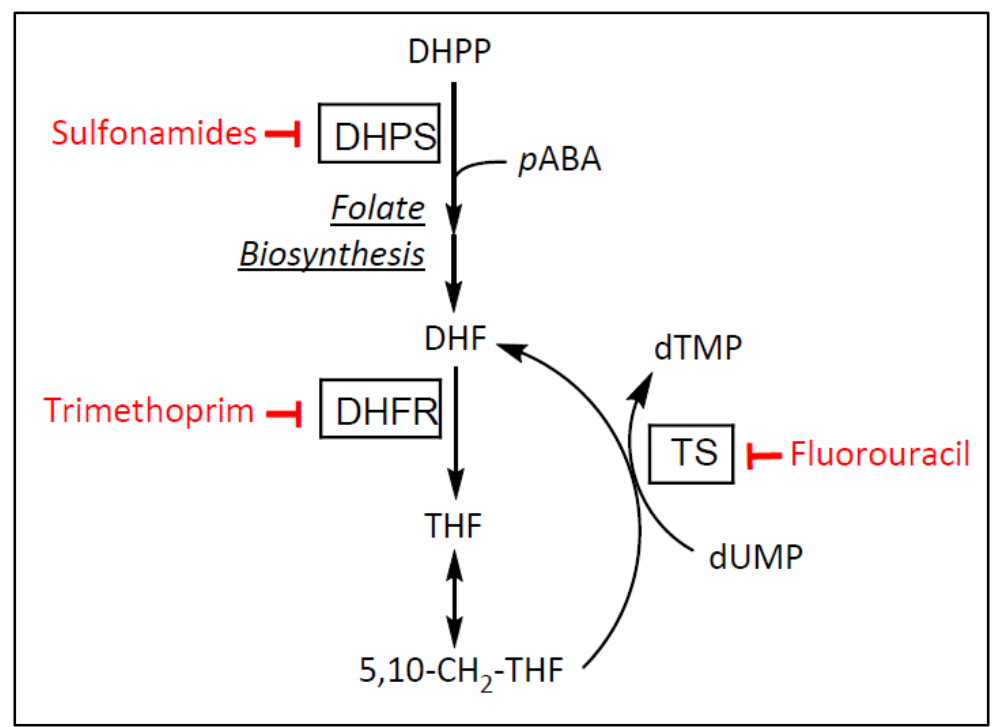

Figure 3-5. Thymidylate synthase is closely linked to the FBP. $\mathrm{TS}=$ Thymidylate synthase . 
Six other chemotherapeutic agents among the secondary confirmed hits also disrupt nucleic acid metabolism as part of their MOA. These secondary hits were decitabine, azacytidine, zebularine, bleomycin, cladribine, and cisplatin (Table 3-2). The folate cycle is closely networked with several metabolic reactions related to DNA metabolism and could potentially be engaged by these chemotherapies. The remaining validated hits from the Bioactives library include STATTIC, BAY 11-7082, and pifithrin. The targets of these chemotherapies are better studied and understood in mammalian systems, and the potential targets in bacteria are unclear. Although these established therapies have a confirmed effect on human targets, selectivity towards the target enzymes in pathogenic species can be achieved through SAR.

Five compounds were validated hits from the Lead-Like library and one compound was a validated hit from the Small Fragment library. The chemical scaffolds of these six hits had no previously reported characterization as anti-infective agents (Table 3-2). The MICs of these hits were not substantially altered by $p$ ABA supplementation, but methionine shifted the MIC of nearly all of these compounds more than two-fold. These compounds represent potential new antimetabolites that may have exciting new MOAs against $E$. coli.

\section{Conclusions and Future Perspectives}

The rise of multi-drug resistant infections has led to a critical need for new and druggable antibiotic targets. There are several unique candidate enzymes both within the FBP and in closely related pathways in bacteria. The screening campaign carried out in this study was intended to detect new small molecule scaffolds with an antimetabolite MOA against E. coli. Growth inhibition in minimal media was measured within the primary HTS, and then antimetabolite effects closely related to folate biosynthesis by testing for MIC "rescue" with either $p$ ABA or methionine were identified during secondary validation. Although the hit rate for GN whole cell screening is often very low and the MOAs of lead compounds are not immediately apparent, the resulting hits from whole cell screens have already met crucial checkpoints in that the molecules are GN permeable and achieve adequate residence time to exert a growth inhibitory effect. For these reasons, a general shift towards phenotype-based screening has been observed among other drug discovery campaigns (Payne 2007, Zlitni 2013). The HTS presented in this chapter initiated the approach of screening whole cell GN bacteria in a nutrientrestricted environment in the Lee laboratory. Similar screening approaches are ongoing against recently developed compound libraries and other GN species with the hopes of continually acquiring GN-effective antimetabolites

A total of nineteen validated hits were obtained from the nutrient-restricted HTS presented in this study. Most of these hits originated from either the Bioactives library of known biologically active molecules with diverse MOAs as well as the Lead-Like library, which was the most extensive library tested consisting of over 10,000 compounds. The Bioactives library served as an ideal molecule set to pilot the primary HTS. The diverse collection of antibiotics from several classes including the antifolates 
allowed for validation of the HTS methods and the metabolic rescue approach (Figure 32). Interestingly, various non-antibiotic hits passed secondary validation including inhibitors of thymidylate synthase and other inhibitors of DNA synthesis as well as STATTIC and pifithrin (Table 3-2). These therapies serve as candidates for drug repurposing as antibiotics, which is a drug discovery method accruing interest in recent years (Farha 2019). A significant advantage of drug repurposing is the fact that the drugs of interest are already extensively characterized for the efficacy, safety, and metabolic profile in humans. This can greatly expedite the clinical approval process.

In addition to the secondary hits originating from the Bioactives collection, five validated hits originated from the Lead-Like library, which was the largest library screened, and one validated hit originated from the Small Fragment collection. None of these molecules have previously reported biological activity and their MOA against $E$. coli is unknown. The fact that $p \mathrm{ABA}$ does not shift the MIC of any of these compounds suggests DHPS and $p$ ABA metabolism are likely not targeted directly by these molecules. Methionine supplementation, however, imparted a significant shift in the MIC for all five Lead-Like compounds (Table 3-2). This suggests these compounds may have an antimetabolite MOA related to the biosynthesis of methionine. Investigation of these compounds is continued in the next chapter. 


\section{CHAPTER 4. DISCOVERY AND DEVELOPMENT OF THIOACETAMIDE- LINKED 1,2,3-TRIAZOLES TARGETING CYSTEINE SYNTHASE A}

\section{Introduction}

\section{Cysteine metabolism in microorganisms}

Similar to folate biosynthesis, the cysteine biosynthetic pathway in bacteria is unique from humans yet necessary for essential cell metabolites. Cysteine is an essential metabolite with important cellular roles common to all domains of life. Cysteine is one of the twenty standard amino acids found in proteins and aids in secondary structure and stability through the formation of disulfide bonds. Additionally, cysteine can be found in the active site of several enzymes as it is a strong nucleophile when oxidized. This amino acid and various sulfur-containing derivatives, such as glutathione and taurine, can act as an antioxidant using the thiol group to participate in redox reactions. Cysteine is also a precursor for a variety of other metabolites including methionine, $S$-adenosylmethionine, FeS clusters, thiamine, and coenzyme A (Mazumder 2016, Mozzarelli 2011).

Although cysteine has universal importance among living organisms, the biosynthetic routes to cysteine between higher eukaryotes and microorganisms are distinct. Higher eukaryotic species can either acquire cysteine through the diet or the transsulfuration pathway, wherein the sulfur group and peptide backbone are derived from methionine and serine, respectively (Griffith 1987). Prokaryotes, lower eukaryotes, and plants have a unique biosynthetic route to cysteine that occurs in two steps (Figure 4-1). First $O$-acetylserine (OALS) is formed from serine by serine acetyltransferase (SAT). Cysteine synthase A (CysK) then mediates a reaction between OALS and sulfide to form cysteine (Rabeh 2004). Sulfide, in this case, is derived from the reductive sulfate assimilation pathway (RSAP) instead of methionine (Sekowska 2000).

The metabolic pathways responsible for cysteine biosynthesis and sulfate assimilation are tightly regulated by the cellular concentrations of various intermediates and products of these pathways. In the absence of OALS, CysK is inhibited by the Cterminal tail of the upstream enzyme SAT (Campanini 2005). Furthermore, the CysK product cysteine inhibits SAT. A high cellular pool of OALS results in the spontaneous rearrangement of OALS to $N$-acetyl-L-serine, which induces the sulfate assimilation pathway (Kredich 1992).

Downstream of cysteine biosynthesis and the RSAP, cysteine acts as a precursor for homocysteine, which feeds into the methyl cycle and the folate cycle in both prokaryotes and eukaryotes (Figure 4-1). Methionine synthase produces methionine from homocysteine and represents a juncture between two metabolically significant pathways: the methyl cycle and the folate cycle (Ferla 2014). These two pathways have indispensable roles in protein biosynthesis, DNA biosynthesis and repair, and methylation reactions. 


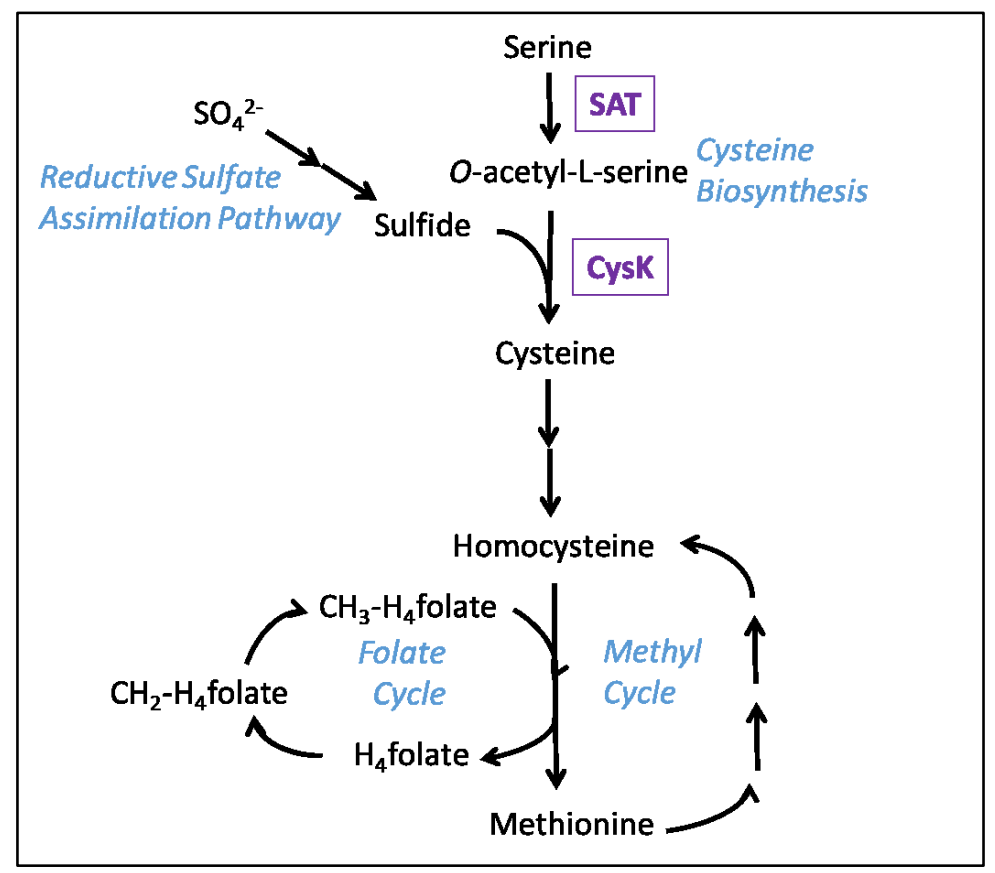

Figure 4-1. Cysteine metabolism and related pathways in microorganisms. $\mathrm{SAT}=$ serine acetyl transferase $;$ CysK $=$ cysteine synthase A. 


\section{Cysteine and virulence in pathogenic microbes}

In addition to cysteine's role in protein structure and several fundamental cell processes, this amino acid also has well-established roles in virulence among several microbial species. The antioxidant properties of cysteine and its derivatives counteract redox stress from the host during infection (Bornemann 1997, Mori 2015). $S$.

typhimurium has heightened susceptibility to certain antibiotics in the absence of the cysteine biosynthetic operon, and the differentiation of swarm-cells requires cysteine biosynthesis (Turnbull 2008). RSAP genes in mycobacteria are upregulated in conditions of oxidative stress and nutrient deprivation, which mimics the conditions encountered during the latent phase of tuberculosis infection (Hatzois 2011). Cysteine is the primary low molecular weight antioxidant in various protozoan parasites (Nozaki 2005). In $E$. histolytica, cysteine has well-established roles in attachment, proliferation, and motility (Mori 2015).

\section{Current antibiotic drug discovery efforts towards CysK}

The unique biosynthetic route to cysteine among microorganisms as well as the roles of cysteine and sulfur assimilation in virulence suggests cysteine biosynthesis is a favorable target for new antimicrobial development. The available literature indicates drug discovery efforts towards CysK as early as the 1970s (Kredich 1975). Kredich and coauthors revealed the ability of 1,2,4-triazole to inhibit the growth of S. typhimurium through interaction with the CysK enzyme. It was demonstrated that several enzymes within a regulon for the RSAP were downregulated in response to 1,2,4-triazole treatment. Additionally, it was determined that the CysK enzyme could form a false product between the triazole and the native CysK substrate OALS. The authors developed the intriguing hypothesis that rather than inhibiting CysK function directly, the triazoles contributed to growth inhibition through depleting cellular pools of OALS, which is necessary for induction of the RSAP pathway (Kredich 1975). These effects were later confirmed by showing dose-dependent reduction of RSAP genes in response to 1,2,4-triazole treatment in S. typhimurium (Turnbull 2008).

Interest in CysK as an antimicrobial target gained stamina at the turn of the century. The CysK enzymes of several pathogenic species have been investigated structurally and evaluated for inhibitor development including E. histolytica (Nagpal 2012, Mori 2015), S. typhimurium (Spyrakis 2013, Magalhães 2018), M. tuberculosis (Jean Kumar 2013, Poyraz 2013), H. influenzae (Salsi 2010, Amori 2012), and T. vaginalis (Singh 2013, Westrop 2006). In contrast to the false-product mechanism of 1,2,4-triazole, the majority of the more recently established CysK inhibitor campaigns generate molecules that directly inhibit the enzyme similarly to the C-terminal tail of SAT (Campanini 2005, Salsi 2010).

Within this chapter, thioacetamide-linked 1,2,3-triazole (TAT) hits from the metabolic screen in Chapter 3 are characterized as new antimicrobial agents targeting CysK in the GN pathogen E. coli. A comprehensive set of experimental approaches are 
employed to confirm the putative target as well as the molecular MOA. These studies present a novel TAT series with a promising new antimetabolite MOA against clinically significant strains of GN bacteria.

\section{Materials and Methods}

\section{Spontaneous mutant generation and genomic sequencing}

ATCC 2511 agar plates were prepared containing each of the three validated hit compounds at $100 \mu \mathrm{g} / \mathrm{mL}$. Each of these plates was inoculated with $3 \times 10^{8} \mathrm{CFU}$ of $E$. coli K12 MG1655 in $1 \mathrm{~mL}$ M9 media, which was spread evenly over the agar and allowed to dry. The plates were incubated at $37^{\circ} \mathrm{C}$. Colonies were apparent but very faint after 24 hours, so the plates were allowed to incubate another day. By 48 hours, adequately sized colonies were visible. Five colonies for each hit compound were used to inoculate M9 containing the respective compounds at $100 \mu \mathrm{g} / \mathrm{mL}$. The parent $E$. coli was also used to inoculate M9 liquid media containing the compounds. Most of the selected mutants survived and grew in the compound-containing media, while the parent E. coli did not grow.

A single colony of each mutant was used to inoculate overnight cultures in M9 containing $100 \mu \mathrm{g} / \mathrm{mL}$ of the compound used to generate each mutant. The Qiagen DNeasy Blood and Tissue Kit was used to extract genomic DNA from each mutant. The genome sequence of each strain was determined through HiSeq Illumina sequencing. CLC Genomics Workbench was used to map the resulting reads to the E. coli $\mathrm{K} 12$ MG1655 reference genome (GenBank U00096.3) using CLC Genomics Workbench. Fixed ploidy variant detection was used to determine the spontaneous mutations present.

\section{Susceptibility testing}

MIC testing was performed as previously described in Chapter 2.

\section{Structural similarity search based on hit compounds}

A nearest neighbor structure search was performed on the entire St. Jude compound collection with structures from an initially identified set of active compounds, as queries. The MDL Public Keys (Durant 2002), implemented in Pipeline Pilot (Dassault Systemes BIOVIA 2018), were used as molecular fingerprints for the nearest neighbor search. A Tanimoto Coefficient cutoff of 0.8 was used to limit the number of nearest neighbors identified from the search. 


\section{EcCysK heterologous expression and purification}

The CysK construct encoding the cysK gene (UniProt P0ABK5) for heterologous protein expression and purification was cloned into the NdeI-NheI cloning site of the pET-28a $(+)$ vector by GenScript. The N-terminal region consisted of a $6 \mathrm{X}$-Histidine tag with a thrombin cleavage site between the histidine tag and the cysK gene. This plasmid was transformed into chemocompetent BL21(DE3) E. coli cells. Growth in the presence of $50 \mu \mathrm{g} / \mathrm{mL}$ Kanamycin, as well as sequencing via the T7 primer set, confirmed successful transformation of the plasmid and correct sequence of the construct.

A single colony of this host strain was used to inoculate an overnight culture in LB with Kanamycin. The next day, this culture was diluted 1:100 in 4 liters of $\mathrm{LB} / \mathrm{Kan} 50$ and allowed to grow to an $\mathrm{OD}_{600}$ of 0.5 . IPTG was added to a final concentration of $1 \mathrm{mM}$, and the culture was incubated overnight at $18^{\circ} \mathrm{C}$. The bacteria was then pelleted at $3,700 \mathrm{RCF}, 10$ minutes, $4^{\circ} \mathrm{C}$ and the pellets were stored at $-20^{\circ} \mathrm{C}$ for up to two weeks.

For every liter of bacteria, $50 \mathrm{~mL}$ of lysis buffer was prepared. This buffer consisted of Buffer A (50 mM Tris- $\mathrm{HCl}, 500 \mathrm{mM} \mathrm{NaCl}, 5 \mathrm{mM}$ imidazole, $\mathrm{pH}$ 8.0), 1 protease pellet (Roche 11-836-170-001), and $450 \mathrm{mg}$ of chicken egg lysozyme. The pellets were thawed and resuspended by pipetting into this buffer until the bacterial pellets were in solution. This was followed by sonication at $100 \%$ amplitude for 3 minutes followed by a 1 -minute rest. The sonication was repeated two more times. The sonication time was increased by another 3 minutes for every additional liter of bacteria included.

After the sonication, the lysate was centrifuged at 20,000 RCF, 20 minutes, and $4^{\circ}$ C. The supernatant was retained and filtered through a $0.22 \mu \mathrm{M}$ filter. The lysate was then run over two stacked His Trap HP $5 \mathrm{~mL}$ columns at $0.5 \mathrm{~mL} / \mathrm{minute}$ using the AKTA Pure purification system. The His-tagged target protein was eluted using a continuous gradient of $0-100 \%$ Buffer B (Same formulation as Buffer A but with an imidazole concentration of $500 \mathrm{mM}$ ) run at $0.5 \mathrm{~mL} /$ minute for 2 hours. Fractions with elevated UV spectra were analyzed by denaturing gel electrophoresis and total protein staining to ensure purity and correct size of the protein. Fractions that were at least $95 \%$ pure were pooled and ran over a HiPrep 26/60 Sephacryl S-300 HR column at $0.5 \mathrm{~mL} /$ minute in 50 $\mathrm{mM}$ HEPES, $150 \mathrm{mM} \mathrm{NaCl}, \mathrm{pH}$ 7.6. The resulting fractions that showed elevated UV spectra were again analyzed by total protein staining. Fractions containing CysK protein were pooled, aliquoted and flash frozen. The protein was stored at $-80^{\circ} \mathrm{C}$ until ready for use.

\section{Spectroscopic studies with CysK}

To ensure the PLP cofactor was fully incorporated into the CysK protein, CysK was allowed to gently rotate with $0.5 \mathrm{mM}$ PLP in $25 \mathrm{mM}$ HEPES, $150 \mathrm{mM} \mathrm{NaCl}$, pH 7.6 for 30 minutes. Since PLP exhibits its own spectroscopic profile, exogenous cofactor 
was removed by then equilibrating CysK into the same buffer lacking PLP by washing with equivalent volumes three times in a 10,000 MWCO centricon. OALS was prepared in the same buffer, while all inhibitory molecules were prepared in DMSO. Final assay concentrations were $100 \mu \mathrm{M}$ CysK and $500 \mu \mathrm{M}$ for both substrate and inhibitors. The DMSO concentration for all samples was $1.25 \%$.

\section{RNA-seq studies}

Two medias were used for the RNA-seq analysis. M9 is the standard M9-based media ATCC2511 used in previous experiments, and M9T has the same media composition with taurine as the sulfur source. To make M9T, magnesium sulfate was omitted, and magnesium chloride was added at a final concentration of $2 \mathrm{mM}$. Taurine was added at a final concentration of $0.25 \mathrm{mM}$ (Gyaneshwar 2005).

A single colony of $E$. coli $\mathrm{K} 12 \mathrm{MG} 1655$ was picked into $5 \mathrm{~mL}$ M9 broth and grown overnight at $37^{\circ} \mathrm{C}, 225 \mathrm{RPM}$ shaking. The next day, two $200 \mu \mathrm{L}$ samples were taken from the overnight culture and pelleted at $3700 \mathrm{xG}$ for 10 minutes. The supernatant was removed and one sample was washed in $1 \mathrm{~mL} \mathrm{M9}$ while another sample was washed in $1 \mathrm{~mL}$ M9T. The samples were pelleted as before and the supernatant removed. The samples were resuspended in $100 \mu \mathrm{L}$ of their respective media and added to $100 \mathrm{~mL}$ of M9 or M9T. The cultures were allowed to grow to mid-log at $37^{\circ} \mathrm{C}, 225 \mathrm{RPM}$ shaking. Five $\mathrm{mL}$ of mid-log culture was added to $50 \mathrm{~mL}$ conical tubes containing $50 \mu \mathrm{L}$ DMSO or $50 \mu \mathrm{L}$ of $1.56 \mathrm{mg} / \mathrm{mL} 3866$ to achieve a final concentration of $15.6 \mu \mathrm{g} / \mathrm{mL}$. The cultures were allowed to drug for 30 minutes at $37^{\circ} \mathrm{C}, 225 \mathrm{RPM}$ shaking. Afterward, 10 mL RNAprotect Bacteria Reagent was immediately added and mixed with the drugged cultures and pelleted at 5,000xG for 10 minutes. The supernatant was discarded and the pellets were flash frozen. Each group (drugged and undrugged in either M9 or M9T) was prepared in triplicate from three different colonies.

For RNA extraction, pellets of E. coli K12 were re-suspended in $100 \mu \mathrm{L}$ of 1 $\mathrm{mg} / \mathrm{mL}$ lysozyme and incubated at $37^{\circ} \mathrm{C}$ for 10 minutes with 5 seconds vortex every 2 minutes. Subsequent steps were carried out using the RNeasy extraction kit (Qiagen) according to protocol. RNA was eluted in $40-50 \mu \mathrm{L}$ of supplied elution buffer. Residual genomic DNA contamination was depleted using RNA Clean and Concentrator kit (Zymo Research) following the manufacture's protocol. Samples were eluted in $50 \mu \mathrm{L}$ of DNase/Rnase-Free water. Quality of RNA samples was measured using an Agilent 2100 Bioanalyzer and RIN values were all > 7.0.

The ScriptSeq ${ }^{\mathrm{TM}}$ v2 RNA-seq Library Preparation Kit was used to prepare the RNA-seq library. Total RNA was sequenced as 100 bp paired end reads using Illumina HiSeq technology. Ribosomal RNA sequences were filtered out using BBDuk $\{$ https://jgi.doe.gov/data-and-tools/bbtools/\}. The resulting reads were aligned to the reference $E$. coli str. K-12 substr. MG1655 genome (NCBI) using Rockhopper $\{23716638\}$ to generate the gene counts. Differential gene expression analysis was analyzed using the DESeq2 analysis package $\{25516281\}$ in the $\mathrm{R}$ statistical computing 
environment from the raw gene count data of the protein coding sequences generated from Rockhopper.

\section{Results and Discussion}

Among the validated hits from the HTS in Chapter 3, three molecules originating from the Lead-Like library were selected for further lead development and assigned lab identification numbers. These compounds shared a 2-((1H-1,2,3-triazol-5-yl)thio)-N(phenyl)acetamide scaffold (thioacetamide-linked 1,2,3-triazoles or TATs), and their growth inhibition was antagonized by methionine (Table 3-2 and Figure 4-2). These qualities indicated the possibility of a common MOA between the three molecules.

\section{Initial characterization of the TAT hits and target determination}

The three Lead-Like hits 3866, 3865, and 3864 were synthesized in-house and their MICs confirmed. The newly-synthesized compounds maintained growth inhibition against $E$. coli $\mathrm{K} 12$ and their MIC could still be antagonized by methionine supplementation (Table 4-1). To obtain preliminary indications towards the target of the TATs, E. coli spontaneous mutants were raised separately against the three compounds on M9 agar with inhibitor concentrations equivalent to $\sim 30-60 \mathrm{x}$ the MIC. The resulting resistance frequencies were in the range of $1-3 \times 10^{-7} \mathrm{CFU}$.

For each compound, five colonies were selected from the spontaneous mutant plates to screen for cross-resistance to the other hit compounds. All five colonies selected for 3864 and 3865 were successfully cultivated and maintained resistance. Only two of the five colonies selected for 3866 continued to grow in the presence of 3866 . All 12 mutants were resistant to all three TAT Lead-Like hits, indicating a shared MOA (Table 4-2).

To investigate the mechanism of resistance to the TAT inhibitors, genomic DNA was extracted from the twelve spontaneous mutants and sequenced. The strains tested had nonsynonymous mutations in the cysK gene, which encodes cysteine synthase A (CysK) (Table 4-3). The nearly universal presence of cysK mutations in the E. coli spontaneous mutants was a robust initial indication that CysK is targeted by the TATs.

\section{Modeling of observed resistance mutations on the $E$. coli CysK enzyme}

Given the fact that nearly all mutants had nonsynonymous mutations in the CysK protein, it seemed possible that the lead molecules may interact directly with this enzyme. CysK is a pyridoxal-L-phosphate (PLP) dependent fold type II enzyme with a substrate binding channel between the $\mathrm{N}$ - and C-terminal domains (Mozzarelli 2011). Within this channel is an invariant lysine that forms an internal aldimine linkage with PLP, which switches to an external linkage with OALS as part of its catalytic mechanism. PLP is 


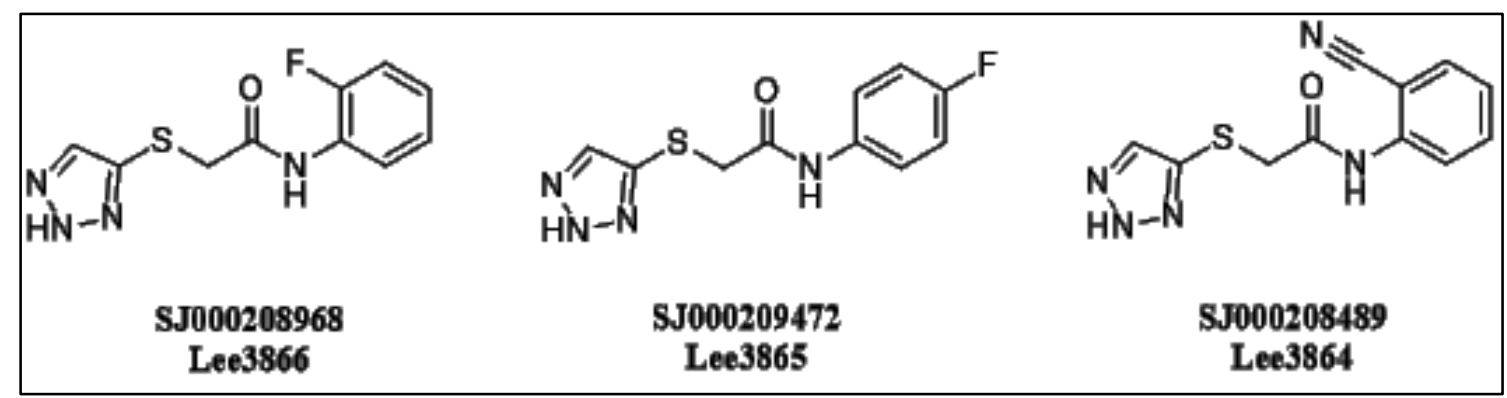

Figure 4-2. Thioacetamide-linked 1,2,3-triazole hits from the metabolic screen. Original St. Jude numbers and assigned lab identification numbers are shown.

Table 4-1. Preliminary MICs and resistance frequency of the TAT hits.

\begin{tabular}{|c|c|c|c|c|}
\hline Lee No. & Structure & $\begin{array}{c}\text { E. coli K12 } \\
\mu \mathrm{g} / \mathrm{mL} \text { MIC90 }\end{array}$ & $\begin{array}{c}\text { L-methionine } \\
\text { MIC Shift }\end{array}$ & $\begin{array}{l}\text { Resistance } \\
\text { Frequency }^{\mathrm{b}}\end{array}$ \\
\hline 3866 & & 1.6 & 2 & $1.3 \times 10^{-7}$ \\
\hline 3865 & & 3.1 & 2 & $2.7 \times 10^{-7}$ \\
\hline 3864 & & 3.1 & 4 & $2.8 \times 10^{-7}$ \\
\hline
\end{tabular}

${ }^{\text {a } M e t h i o n i n e ~ s h i f t ~ i s ~ a ~ r a t i o ~ o f ~ t h e ~ M I C ~ i n ~ t h e ~ p r e s e n c e ~ o f ~} 20 \mu \mathrm{g} / \mathrm{mL}$ L-methionine over the MIC in M9 with no supplements.

${ }^{\mathrm{b}}$ Determined in the presence of $100 \mu \mathrm{g} / \mathrm{mL}$ of each compound. 
Table 4-2. MIC shifts of spontaneous mutants at 24 hours.

\begin{tabular}{|c|c|c|c|c|c|c|c|c|c|c|c|c|}
\hline \multirow[b]{2}{*}{ Compound } & \multicolumn{5}{|c|}{3864 Mutant No. } & \multicolumn{5}{|c|}{3865 Mutant No. } & \multicolumn{2}{|c|}{3866 Mutant No. } \\
\hline & 1 & 2 & 3 & 4 & 5 & 1 & 2 & 3 & 4 & 5 & 1 & 4 \\
\hline 3864 & 64 & 64 & 64 & $>64$ & 64 & 64 & 64 & 64 & 64 & 64 & $>64$ & $>64$ \\
\hline 3865 & $>64$ & $>64$ & $>64$ & $>64$ & 64 & $>64$ & $>64$ & $>64$ & $>64$ & 64 & $>64$ & $>64$ \\
\hline 3866 & $>64$ & $>64$ & $>64$ & $>64$ & $>64$ & $>64$ & $>64$ & $>64$ & $>64$ & 64 & $>64$ & $>64$ \\
\hline SMX & - & - & - & - & - & - & - & - & - & - & - & - \\
\hline TMP & - & 2 & - & - & - & - & - & 2 & - & 2 & 2 & - \\
\hline CAM & - & - & 0.5 & - & - & - & - & - & 0.5 & - & - & 0.5 \\
\hline KAN & - & - & - & - & 2 & - & 2 & 2 & - & 2 & 2 & - \\
\hline
\end{tabular}

$\mathrm{SMX}=$ Sulfamethoxazole, $\mathrm{TMP}=$ Trimethoprim, $\mathrm{CAM}=$ Chloramphenicol, $\mathrm{KAN}=$ Kanamycin. Dashes represent the absence of a detectable MIC shift (Mutant MIC/WT $\mathrm{MIC}=1)$. 
Table 4-3. Chromosomal mutations in spontaneous $E$. coli K12 mutants raised against the TATs.

\begin{tabular}{|c|c|c|c|c|c|c|c|c|c|c|c|c|c|c|c|}
\hline \multirow[b]{2}{*}{ Position $^{\mathrm{a}}$} & \multirow[b]{2}{*}{ Mutation Type ${ }^{\text {b }}$} & \multirow{2}{*}{$\begin{array}{l}\text { Residue } \\
\text { Change }\end{array}$} & \multirow[b]{2}{*}{ Gene } & \multicolumn{12}{|c|}{ Mutant $^{\mathrm{c}}$} \\
\hline & & & & 3864-1 & $3864-2$ & 3864-3 & $3864-4$ & $3864-5$ & $3865-1$ & $3865-2$ & $3865-3$ & $3865-4$ & $3865-5$ & 3866-1 & $3866-4$ \\
\hline 686073 & $\mathrm{SNV}, \mathrm{A} \rightarrow \mathrm{C}$ & ND & gltI & & & & & & & & & & & $\mathrm{x}$ & \\
\hline 987494 & $\mathrm{SNV}, \mathrm{T} \rightarrow \mathrm{G}$ & ND & $\operatorname{asn} S$ & & & & & & & & & $\mathrm{x}$ & & & \\
\hline 1207789 & $\mathrm{SNV}, \mathrm{C} \rightarrow \mathrm{G}$ & ND & $t f a E$ & & & & & & & & & $\mathrm{x}$ & $\mathrm{x}$ & & \\
\hline 2532518 & $\mathrm{SNV}, \mathrm{C} \rightarrow \mathrm{A}$ & P37H & cys $K$ & & & & & & & & & $\mathrm{x}$ & & & \\
\hline 2532538 & $\mathrm{SNV}, \mathrm{C} \rightarrow \mathrm{G}$ & R44G & cys $K$ & & & & & & & & & & $\mathrm{x}$ & & \\
\hline 2532616 & $\mathrm{SNV}, \mathrm{A} \rightarrow \mathrm{T}$ & S70C & cys $K$ & & & & $\mathrm{x}$ & & $\mathrm{x}$ & $\mathrm{x}$ & & & & & \\
\hline 2532620 & $\mathrm{SNV}, \mathrm{G} \rightarrow \mathrm{C}$ & G71R & cys $K$ & & & & & & & & & & & & $\mathrm{x}$ \\
\hline 2533321 & $\mathrm{SNV}, \mathrm{C} \rightarrow \mathrm{A}$ & R305S & $\operatorname{cys} K$ & $\mathrm{x}$ & $\mathrm{x}$ & & & & & & $\mathrm{x}$ & & & & \\
\hline 2533322 & $\mathrm{SNV}, \mathrm{G} \rightarrow \mathrm{T}$ & R305L & cysK & & & $\mathrm{x}$ & & & & & & & & & \\
\hline 2533325 & $\mathrm{SNV}, \mathrm{A} \rightarrow \mathrm{T}$ & Y306F & cys $K$ & & & & & $\mathrm{x}$ & & & & & & & \\
\hline
\end{tabular}

$\mathrm{SNV}=$ Single nucleotide variation $; \mathrm{ND}=$ Not determined.

aPosition in genome is relative to E. coli K12 MG1655 genome (GenBank: U00096.3).

${ }^{b}$ Nucleotide change is shown after mutation type.

${ }^{\mathrm{c}}$ The letter $\mathrm{x}$ indicates presence of mutation. 
restored to the internal aldimine linkage after the addition of sulfide and release of the cysteine product (Schnell 2007).

To investigate the locations of the spontaneous mutations on CysK, the affected residues were mapped on a previously solved structure of E. coli CysK (PDB 5J43) (Figure 4-3). The aminoacrylate intermediate from a structure of $M$. tuberculosis CysK (PDB 2Q3D) was overlayed to study the proximity of the mutations to the substrate. All mutations tracked to the substrate binding channel of CysK (Figure 4-3). Two mutated residues P37 and R44 are located directly next to the invariant lysine K42 involved in PLP binding. S70 and G71 are situated in a conserved loop that interacts with the carboxyl groups of three known ligands of CysK including the SAT C-terminal isoleucine, the aminoacrylate reaction intermediate, and the benzoic acid moiety of a thiazolidine inhibitor of M. tuberculosis cysteine synthase (Poyraz 2013). The roles of R305 and Y306 were less clear from the existing literature, but these residues still track to the interface between the two subunits of CysK where ligand binding occurs.

\section{Homology of resistance mutations in the CysK enzyme of various species}

In order to investigate whether the mutated residues residing within the substrate binding channel are well conserved, an alignment of the CysK primary sequences from ten species was assembled (Figure 4-4). In addition to E. coli, the organisms selected were other GN pathogens as well as various species for which CysK is well studied. Residues and/or regions previously reported as having established roles in catalysis were noted (Dharavath 2017). All six residues altered during spontaneous mutant generation were in sites that were $100 \%$ conserved among several GN species, intracellular parasites, and even the model plant organism Arabidopsis thaliana. Furthermore, all mutated residues except R305 and Y306 were either directly within or proximal to regions involved in ligand binding. The location of mutations in highly conserved regions necessary for substrate interactions suggests the TATs may interfere with the catalytic function of CysK.

\section{Cellular studies further confirm CysK as the target of lead inhibitors}

To further study the role of CysK in growth inhibition by the lead TATs, the $E$. coli BW25113 $\Delta$ cysK JW2407 strain, as well as the parent strain, was obtained from the Keio collection (Table 4-4) (Baba 2006). Growth rate studies revealed that the $\Delta$ cysK strain had a severe growth defect in M9 minimal media, which lacks all twenty amino acids (Figure 4-5A). Supplementation of cysteine to the media restored the growth curve to a trend very similar to the WT strain, indicating that cysteine starvation is responsible for the growth defect in the knockout strain. Addition of 3866 to the M9 media at 16 $\mu \mathrm{g} / \mathrm{mL}$ (10x the E. coli $\mathrm{MIC}$ ) inhibited the growth of the WT strain over the 24 hours that growth was measured, but the addition of cysteine restored WT growth in the presence of inhibitor (Figure 4-5A). The similarities between the phenotypic effect of inhibitor and loss of the cysK gene suggests 3866 may act through inhibiting CysK function, resulting 


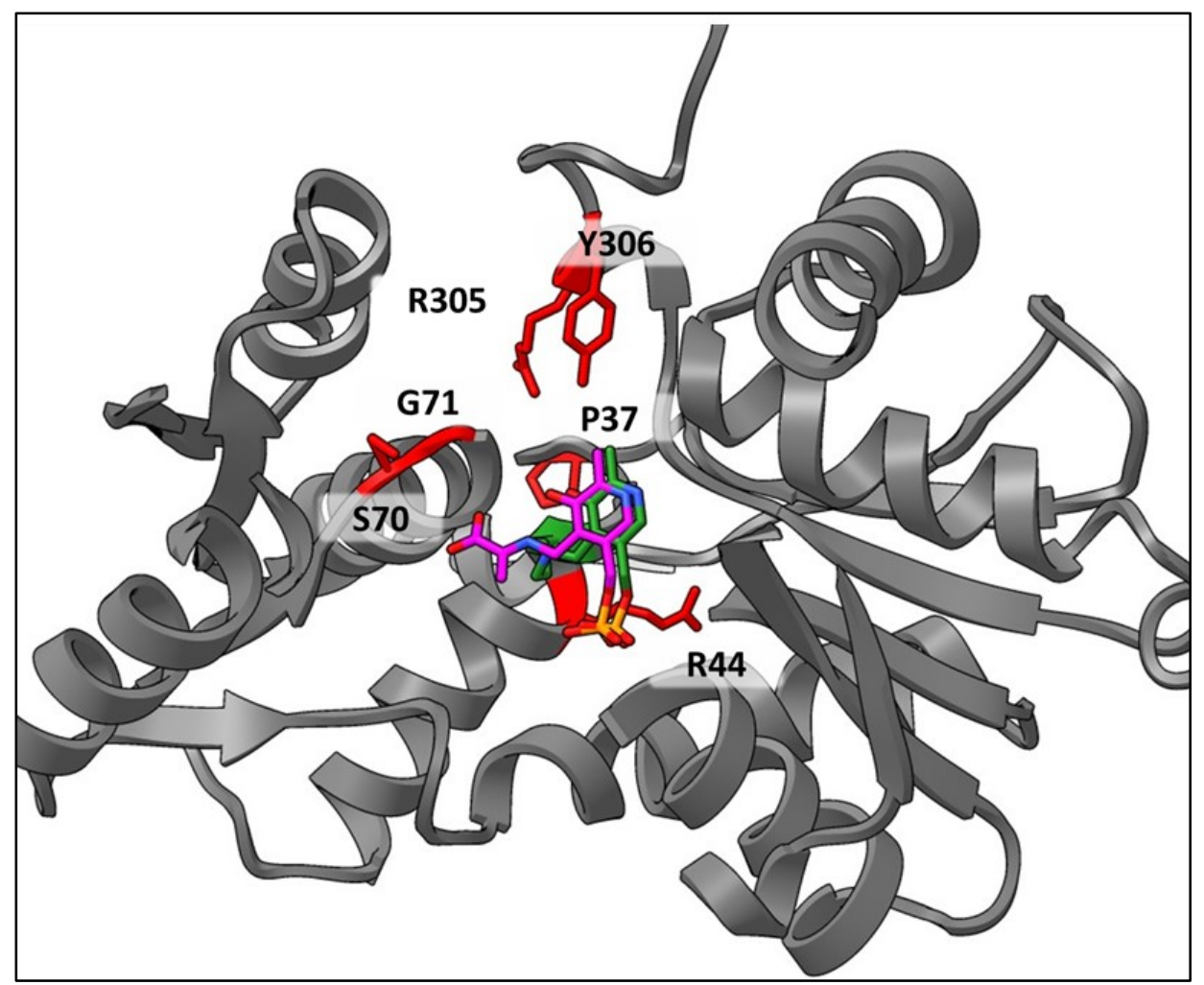

Figure 4-3. Location of spontaneous mutations on $E$. coli CysK.

CysK structure is from PDB 5J43. Lysine 42, which is required for binding with the PLP cofactor, is in green. Residues altered by observed mutations are red and the amino acrylate intermediate from PDB 2Q3D is represented by pink sticks. 


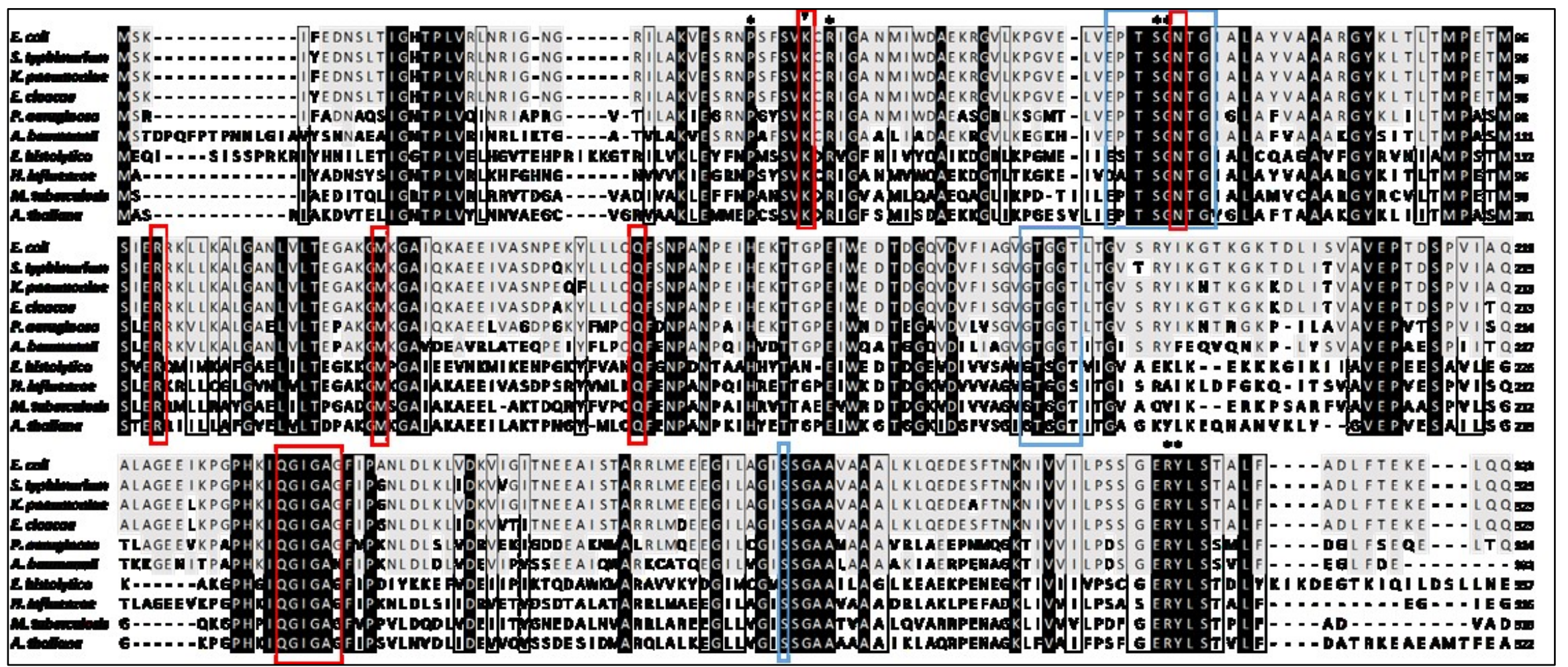

Figure 4-4. Sequence alignment of CysK and location of mutations induced spontaneously for resistance to TATs.

Stars represent location of observed nonsynonymous CysK mutations in spontaneous mutants. The triangle represents the invariant lysine required for interaction with PLP. Red boxes indicate residues known to interact with the SAT C-terminal tail. Blue boxes indicate residues involved in PLP interactions. Residues with established roles in interactions with SAT or PLP were identified in Dharavath 2017. 
Table 4-4. Strains used for characterization of the TATs.

\begin{tabular}{lll}
\hline Strain & \multicolumn{1}{c}{ Characteristics/Genotype } & \multicolumn{1}{c}{ Source } \\
\hline E. coli $\mathrm{K} 12 \mathrm{MG} 1655$ & Control for susceptibility testing & ATCC 700926 \\
E. coli $\mathrm{BW} 25113$ & Parent of knockout strains & (2006 Baba) \\
E. coli JW2407 & BW25113 $\Delta$ tolC, KAN & (2006 Baba) \\
BW pPJ131 empty & BW25113, pPJ131:CBN & This study \\
JW2407 CysK & BW25113 $\Delta$ tolC pPJ131:CysK, KAN KABN $^{\mathrm{R}} \mathrm{CBN}^{\mathrm{R}}$ & This study \\
JW2407 CysK $^{\mathrm{S} 70 \mathrm{C}}$ & BW25113 $\Delta$ tolC $\mathrm{pPJ} 131: \mathrm{CysK}^{\mathrm{S} 70 \mathrm{C}}, \mathrm{KAN}^{\mathrm{R}}, \mathrm{CBN}^{\mathrm{R}}$ & This study \\
\hline
\end{tabular}

Note: $\mathrm{CBN}=$ Carbenicillin .

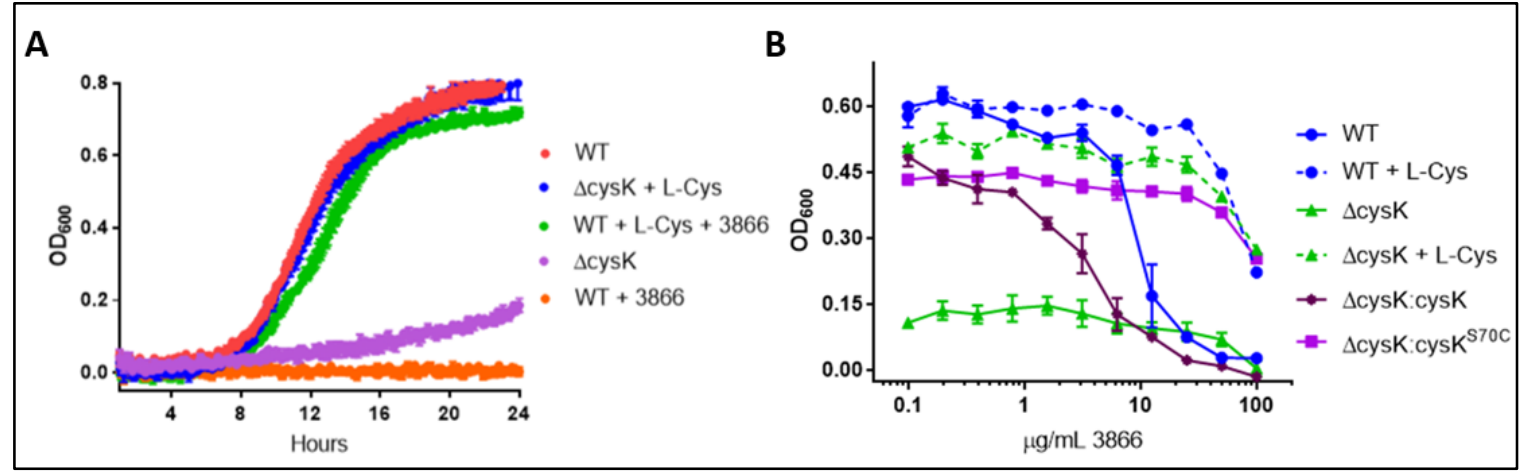

Figure 4-5. Cell-based studies to investigate the putative CysK target.

A. Growth curve study of WT and $\triangle$ CysK E. coli strains in the presence of 3866 and/or L-cysteine. B. Dose-response of 3866 among panel of WT and $\Delta$ CysK strains harboring WT and resistant forms of the cysK gene in the constitutive expression vector pPJ131. 
in cysteine starvation.

To further study target specificity, the cys $K$ gene was cloned into the pPJ131 vector for constitutive expression (Yao 2016). An additional variant of the cys $K$ gene harboring the frequently observed spontaneous mutation S70C was generated in the same plasmid (Tables 4-3 and 4-4). These two plasmids were transformed into the $\Delta \mathrm{CysK}$ strain. The panel of $E$. coli strains was subject to increasing concentrations of 3866 in M9 media and allowed to grow overnight, following methods very similar to a traditional MIC. After 24 hours of growth, the $\mathrm{OD}_{600}$ values were measured (Figure 4-5B). Cysteine supplementation again rescued $E$. coli from growth inhibition. The $\Delta$ CysK strain showed scant growth among all concentrations tested, but in the presence of cysteine showed a growth profile similar to the WT strain, suggesting cysteine auxotrophy. In the presence of both 3866 and L-cysteine, this knockout strain did not appear to have WT susceptibility to 3866 in the absence of supplementation. Addition of the $c y s \mathrm{~K}^{\mathrm{S} 70 \mathrm{C}}$ plasmid to the knockout strain showed a similar resistance profile. These experiments further confirm CysK as the putative TAT target by indicating phenotypic similarities between 3866 treatment and the absence of the CysK. Specificity is further confirmed through the observed correlation between reduced 3866 activity and CysK target modification.

\section{Lead optimization of the TAT series}

A structure-activity-relationship (SAR) study was carried out to see if modifications to the molecular structure of the TATs could improve potency against $E$. coli as well as in vitro pharmacokinetic/pharmacodynamic (PK/PD) properties. For this dissertation, ten molecules in addition to the original three hits were evaluated in terms of activity against $E$. coli and in vitro ADME properties (Figure 4-6 and Table 4-5). These molecules included two previously established CysK-targeted molecules including 1,2,4triazole and a decapeptide representing the last 10 peptides of E. coli SAT (EcP10, NHTFEYGDGI, UnitProt P0A9D4) (Figure 4-6A). The MIC against $E$. coli for 1,2,4triazole was high in comparison to the original TAT hits but detectable, while EcP10 had no detectable activity.

The other eight molecules evaluated within this study are TAT analogs of the original hit 3866 (Figure 4-6B). These molecules were either synthesized in-house or identified using a nearest neighbor structure search to obtain molecules that were already available in the compound library at St. Jude Children's Research Hospital with similar molecular structures to the original TAT hits. MIC detection and in vitro PK/PD studies on the most potent molecules provided valuable information on the SAR of the TAT series. Modifications to the triazole moiety imparted the highest consequence to potency, and the 1,2,3-triazoles were generally more potent than the corresponding scaffolds with a 1,2,4-triazole. Modifications to the thioacetamide linker generally increased the $E$. coli MIC, however the phenyl ring could be modified with little consequence to biological activity. Although none of the analogs were more potent than the original Lead-Like hit 3866 , some in vitro pharmacokinetic properties were improved including solubility and 


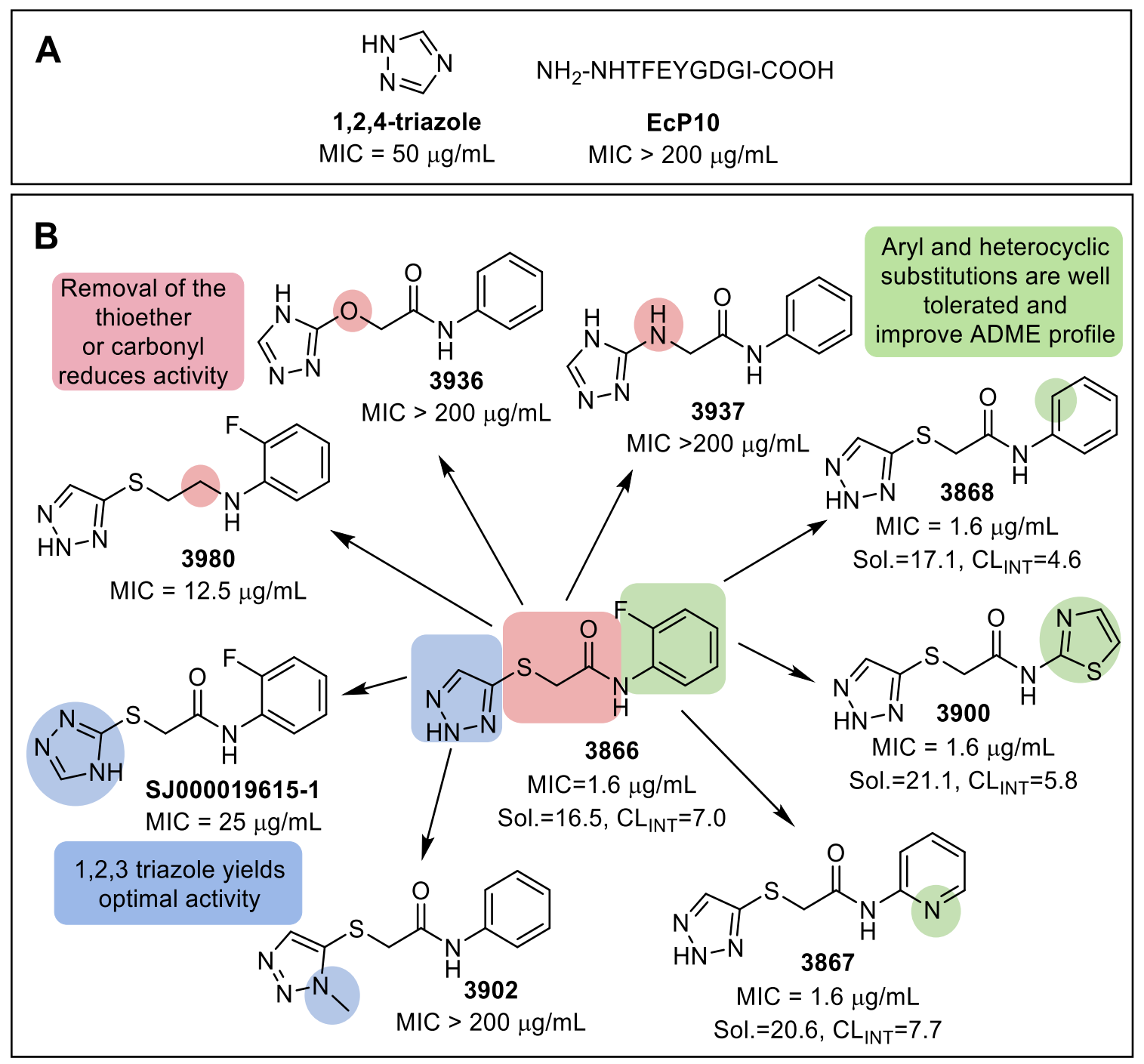

Figure 4-6. TAT molecules representing the SAR study.

For each molecule, the E. coli $\mathrm{K}_{12} \mathrm{MIC}_{90}$ in M9 is shown. For lead inhibitors, solubility (Sol.) is shown in $\mu \mathrm{g} / \mathrm{mL}$ and in vitro clearance rate (CLINT) is shown in $\mathrm{mL} / \mathrm{min} / \mathrm{Kg}$. A. Previously established CysK-targeted molecules. B. Select TAT analogs and their SAR summary. Blue region is the triazole moiety, which is most crucial for potency. Red region is the central portion of the molecule which generally showed a reduction in activity upon modification. The region highlighted in green was amenable to substitutions and could be modified to improve the in vitro PK/PD profile. 
Table 4-5. PK/PD Characterization of Lead TATs.

\begin{tabular}{|c|c|c|c|c|c|}
\hline \multirow[b]{2}{*}{ Compound } & \multirow[b]{2}{*}{$\begin{array}{c}\text { Solubility } \\
(\mu \mathrm{g} / \mathrm{mL})\end{array}$} & \multirow[b]{2}{*}{$\begin{array}{c}\text { Plasma Protein } \\
\text { Binding }(\%)\end{array}$} & \multirow[b]{2}{*}{$\begin{array}{c}\text { Plasma Stability } \\
\text { t1/2 (hr) }\end{array}$} & \multicolumn{2}{|c|}{ Metabolic Stability } \\
\hline & & & & t1/2 (hr) & $\begin{array}{c}\text { CLint } \\
(\mathrm{mL} / \mathrm{Min} / \mathbf{K g})\end{array}$ \\
\hline 3864 & $19.0\left( \pm 1.5^{\mathrm{a}}\right)$ & $78.8( \pm 5.8)$ & $23.0( \pm 2.1)$ & $3.7( \pm 0.30)$ & 5.7 \\
\hline 3865 & $18.5( \pm 2.3)$ & $86.6( \pm 1.0)$ & $36.3( \pm 5.5)$ & $4.7( \pm 0.42)$ & 4.4 \\
\hline 3866 & $16.5( \pm 0.82)$ & $90.5( \pm 1.1)$ & $34.8( \pm 4.9)$ & $3.0( \pm 0.22)$ & 7.0 \\
\hline 3900 & $21.1( \pm 1.9)$ & $76.9( \pm 6.6)$ & $32.1( \pm 5.4)$ & $3.6( \pm 0.34)$ & 5.8 \\
\hline 3868 & $17.1( \pm 0.62)$ & $88.8( \pm 1.3)$ & $35.5( \pm 4.0)$ & $4.5( \pm 0.40)$ & 4.6 \\
\hline 3867 & $20.6( \pm 3.1)$ & $71.2( \pm 9.4)$ & $19.9( \pm 1.9)$ & $2.7( \pm 0.21)$ & 7.7 \\
\hline Carbamazepine & $20.1( \pm 0.79)$ & NT & NT & NT & NT \\
\hline Albendazole & $5.3( \pm 0.22)$ & NT & NT & NT & NT \\
\hline Ranitidine & $27.2( \pm 0.84)$ & NT & NT & NT & NT \\
\hline Verapamil & $39.0( \pm 0.96)$ & $94.2( \pm 0.13)$ & $0.36( \pm 0.0061)$ & $1.5( \pm 0.088)$ & 14.0 \\
\hline
\end{tabular}

Note: $\mathrm{NT}=$ Not tested.

${ }^{a}$ Values in parenthesis represent standard deviation. 
clearance rate in human liver microsomes (Figure 4-6B and Table 4-5).

\section{Antagonism of MIC by metabolites closely related to L-cysteine biosynthesis}

CysK is responsible for producing cysteine, which provides the thiol moiety in methionine and feeds into the methyl cycle (Ferla 2014). This could explain the shift in MIC observed in the presence of methionine. To further validate $\mathrm{CysK}$ as the target using differential media conditions, the MIC was measured in the presence of additional metabolites closely related to cysteine biosynthesis. The 1,2,4-triazole molecule was included in these studies since it is also known to interact with CysK and has a high yet detectable MIC in E. coli of $50 \mu \mathrm{g} / \mathrm{mL}$ (Kredich 1975) (Table 4-6).

Several metabolites closely linked to CysK biosynthesis antagonized the growth inhibitory effect of the triazole-based compounds. These metabolites included OALS, serine, sulfide, and glutathione. A mix of amino acids representing all 20 standard amino acids with the exception of cysteine, serine, and methionine exhibited a mild shift of twofold. The 1,2,4-triazole molecule exhibited a similar pattern of antagonism, suggesting a shared antimetabolite mechanism towards CysK (Table 4-6).

\section{TATs potentiate DHPS inhibitors}

Antimetabolites such as the antifolate antibiotics are known to exhibit a high degree of synergy in combination by inhibiting different enzymes within the same metabolic network simultaneously (Bermingham 2002, Minato 2018). To explore the potential for partner agents that could show synergy and/or potentiation in combination with the TAT series, the $\triangle \mathrm{CysK}$ strain was first tested against a panel of frontline antibiotics and experimental antifolates. Given the fact that folate and cysteine biosynthetic pathways feed into common metabolic cycles such as the folate cycle and methyl cycle, various antifolates were tested against the knockout strain. In addition to SMX and TMP, the pterin-sulfamethoxazole compound 568 was tested as well as MAC173979, which is an inhibitor of $p$ ABA biosynthesis that is known to potentiate other antifolates (Zhao 2016, Thiede 2016). The $\Delta$ CysK strain did indeed show hypersusceptibility to all antifolates tested, as well as increased susceptibility to CAM and a subtle increase in susceptibility to tetracycline (Table 4-7).

To further confirm the potential for synergistic or potentiation effects between the TATs and the antibiotics that were more potent against the $\triangle$ CysK strain, E. coli WT checkerboard MICs were performed between these antibiotics and the lead TAT 3900. The SMX and TMP combination was included as a control and displayed the classic synergy profile commonly observed between these two antifolates (Figure 4-7A). Unfortunately, none of the combinations with 3900 exhibited synergy. However, the two DHPS inhibitors tested, 568 and SMX, were potentiated by 3900 (Figure 4-7B, C). These effects indicate E. coli is more susceptible to DHPS inhibition when the cysteine biosynthetic pathway is disrupted, which further confirms the associations between the 
Table 4-6. Susceptibility testing of TATs in the presence of metabolites related to cysteine biosynthesis.

\begin{tabular}{|c|c|c|c|c|c|c|c|c|c|}
\hline \multirow[b]{2}{*}{ Compound } & \multirow[b]{2}{*}{$\begin{array}{l}\mu \mathrm{g} / \mathrm{mL} \\
\mathrm{MIC}_{90}\end{array}$} & \multicolumn{8}{|c|}{ E. coli BW25113 MIC Shift ${ }^{\mathrm{a}}$} \\
\hline & & L-Cys & GSH & L-Met & L-Ser & OALS & $\mathrm{Na}_{2} \mathrm{~S}$ & $\mathbf{A A}-\mathbf{C M S}^{\mathrm{b}}$ & pABA \\
\hline 3866 & 0.78 & $>256$ & 128 & 2 & 128 & 128 & 8 & 2 & 2 \\
\hline $\begin{array}{c}1,2,4- \\
\text { triazole }\end{array}$ & 50.0 & 64 & 32 & 4 & 64 & 64 & 4 & 2 & 2 \\
\hline EcP10 & $>200.0$ & NT & NT & NT & NT & NT & NT & NT & NT \\
\hline SMX & 0.10 & 0.5 & 2 & 8 & 2 & 2 & 2 & 2 & $>256$ \\
\hline TMP & 0.39 & 0.5 & 1 & 1.0 & 0.5 & 0.5 & 1 & 2 & 1 \\
\hline AMP & 0.78 & 2.0 & 1.5 & 2.0 & 1 & 1 & 2 & 2 & 1 \\
\hline
\end{tabular}

SMX $=$ Sulfamethoxazole; TMP $=$ Trimethoprim; AMP $=$ Ampicillin; GSH $=$ Glutathione.

${ }^{\mathrm{a}} \mathrm{MIC}$ shift is the ratio of averaged MICs from two experiments in supplemented M9 over unsupplemented M9. Dashes represent the absence of a shift of two-fold or higher.

${ }^{\mathrm{b}} \mathrm{AA}-\mathrm{CMS}$ is all twenty standard amino acids except cysteine, methionine, and serine.

Table 4-7. Susceptibility of $E$. coli $\Delta C y s K$ to various antibiotic compounds.

\begin{tabular}{|c|c|c|c|}
\hline \multirow[b]{2}{*}{ Compound } & \multicolumn{2}{|c|}{$\mu \mathrm{g} / \mathrm{mL} \mathrm{MIC}_{90}{ }^{\mathrm{a}}$} & \multirow{2}{*}{$\begin{array}{c}\Delta \text { CysK fold } \\
\text { decrease in MIC }\end{array}$} \\
\hline & BW25113 & $\Delta$ CysK & \\
\hline Ampicillin & 0.78 & 0.78 & 1 \\
\hline 3900 & 3.1 & 50.0 & 0.063 \\
\hline Sulfamethoxazole & 0.098 & 0.024 & 4 \\
\hline MAC173979 & 25.0 & 3.1 & 8 \\
\hline 568 & 3.1 & 0.78 & 4 \\
\hline Trimethoprim & 0.39 & 0.049 & 8 \\
\hline Tetracycline & 0.78 & 0.39 & 2 \\
\hline Chloramphenicol & 3.1 & 0.78 & 4 \\
\hline
\end{tabular}

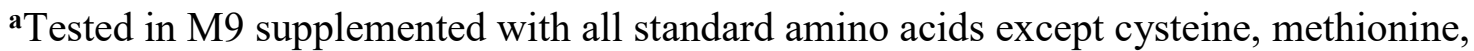
and serine to accommodate the growth of the knockout strain. $\mathrm{MIC}_{90}$ S were determined after 24 hours of growth.

${ }^{\mathrm{b}} \Delta \mathrm{CysK}$ fold decrease in MIC was calculated as a ratio of $\Delta \mathrm{CysK}$ MIC/BW25113 MIC. 

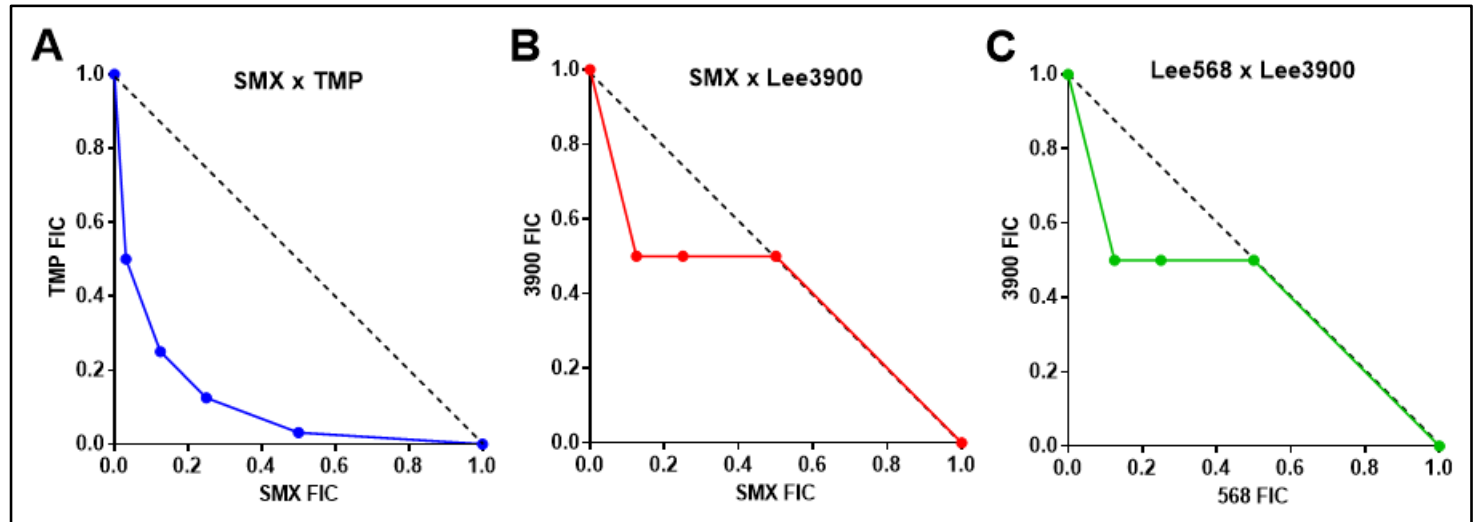

Figure 4-7. 3900 potentiates inhibitors of DHPS.

Fractional inhibitory concentrations (FIC) (MIC of drug A/MIC of drug B) are shown from three checkerboard MIC experiments. A. SMX x TMP. B. SMX x 3900. C. 568 x 3900. $\mathrm{SMX}=$ Sulfamethoxazole; TMP $=$ Trimethoprim. 
folate and cysteine biosynthetic pathways.

\section{TATs maintain activity against antifolate-resistant GN clinical isolates}

The lead TAT compounds 3866, 3900, 3868, and 3867 were tested against a collection of twelve randomly selected GN E. coli and K. pneumoniae clinical isolates from St. Jude Children's Research Hospital with varying antifolate resistance profiles (Table 4-8). These compounds maintained growth inhibitory activity against all strains except one $K$. pneumoniae isolate. Relative to the representative lab strains, in most cases the MIC did not vary more than two-fold. Resistance to either SMX or TMP in each strain did not correspond with increased resistance to the TATs (Table 4-8). This indicates that the MICs detected in lab strains are reflective of MICs against clinical isolates. Furthermore, despite the possibility of interactions with folate biosynthesis detected in the potentiation studies, the TATs are not susceptible to circulating antifolate resistance mechanisms.

\section{In vitro interactions of the TATs with the CysK enzyme}

CysK is a PLP-dependent enzyme, and therefore this enzyme has a unique spectroscopic profile based on the state of the PLP cofactor (Campanini 2005). Spectroscopy has aided other drug discovery campaigns against CysK and could be used to characterize interactions with our TAT series. The absorbance spectra of the apoenzyme shows peak absorbance intensity at $410 \mathrm{~nm}$ which shifts to peak intensity at $466 \mathrm{~nm}$ and shoulder formation at 330nm upon OALS adduct formation (Steiner 2014). Inhibitory peptides mimicking the C-terminal tail of SAT increase the fluorescence intensity of CysK at $510 \mathrm{~nm}$ upon binding (Campanini 2005, Spyrakis 2013). To compare CysK interactions between triazole-containing compounds and inhibitory peptides, the 1,2,4-triazole molecule and EcP10 were included in the spectroscopic studies as controls in addition to 3866 .

The scanning absorbance profile of recombinant E. coli CysK (EcCysK) exhibited the expected profile of PLP-dependent enzymes with both internal and external aldimine linkage states of PLP (Figure 4-8A). Addition of OALS shifted the $410 \mathrm{~nm}$ peak to 466 $\mathrm{nm}$ and increased absorbance of the $330 \mathrm{~nm}$ shoulder. Addition of 3866, 1,2,4-triazole, and EcP10 alone did not cause dramatic changes to the absorbance spectra, however EcP10 decreased the absorbance at 410nm (Figure 4-8A). The fluorescence intensity spectrum was also monitored to see if any changes in the $510 \mathrm{~nm}$ emission were apparent. As expected, EcP10 significantly increased the fluorescence intensity at $510 \mathrm{~nm}$ while OALS decreased the fluorescence intensity relative to the apoenzyme (Figure 4-8B). In contrast to the peptide inhibitor, 3866 and 1,2,4-triazole had a negligible effect on fluorescence.

Although the TAT 3866 and the 1,2,4-triazole molecule exhibited very little change to the absorbance and fluorescence spectra of CysK, notable effects were 
Table 4-8. Lead CysK inhibitors are effective against antifolate-resistant GN clinical isolates.

\begin{tabular}{llcccccc}
\hline \multirow{2}{*}{ Isolate } & \multicolumn{7}{c}{$\mathbf{M I C}_{\mathbf{9 0}}(\boldsymbol{\mu} \mathbf{g} / \mathbf{m L})^{\mathbf{a}}$} \\
\cline { 2 - 7 } E. coli & AMK & SMX & TMP & $\mathbf{3 8 6 6}$ & $\mathbf{3 9 0 0}$ & $\mathbf{3 8 6 8}$ & $\mathbf{3 8 6 7}$ \\
BW251 13 & 1.0 & 0.098 & 0.31 & 1.6 & 1.0 & 1.6 & 1.2 \\
EcCI 1 & 6.3 & $>25$ & $>25$ & 1.6 & 1.6 & 1.3 & 1.3 \\
EcCI 2 & 5.2 & 0.39 & 0.49 & 3.5 & 3.5 & 2.0 & 1.6 \\
EcCI 3 & 3.1 & 0.78 & $>25$ & 2.7 & 3.1 & 2.7 & 3.1 \\
EcCI 4 & 3.1 & $>25$ & $>25$ & 1.3 & 1.6 & 1.0 & 1.3 \\
EcCI 5 & 3.1 & $>25$ & $>25$ & 3.1 & 3.1 & 2.6 & 2.6 \\
EcCI 6 & 9.4 & $>25$ & $>25$ & 1.6 & 1.6 & 1.6 & 1.6 \\
K. pneumoniae & & & & & & & \\
ATCC 700603 & 0.78 & $>25$ & 3.1 & 33 & 50 & 25 & 25 \\
KpCI 1 & 1.6 & 0.78 & 0.68 & 15.6 & 25 & 12.5 & 12.5 \\
KpCI 2 & 5.2 & $>25$ & $>25$ & $>100$ & $>100$ & $>100$ & $>100$ \\
KpCI 3 & 1.6 & $>25$ & $>25$ & 12.5 & 25 & 12.5 & 12.5 \\
KpCI 4 & 1.0 & 0.65 & 0.78 & 12.5 & 25 & 12.5 & 12.5 \\
KpCI 5 & 0.78 & $>25$ & $>25$ & 21 & 25 & 16.7 & 16.7 \\
KpCI 6 & 1.0 & $>25$ & $>25$ & 25 & 42 & 25 & 12.5 \\
\hline
\end{tabular}

AMK = Amikacin; SMX = Sulfamethoxazole; TMP $=$ Trimethoprim.

${ }^{\mathrm{a}} \mathrm{MICs}$ are an average of at least three experiments. 


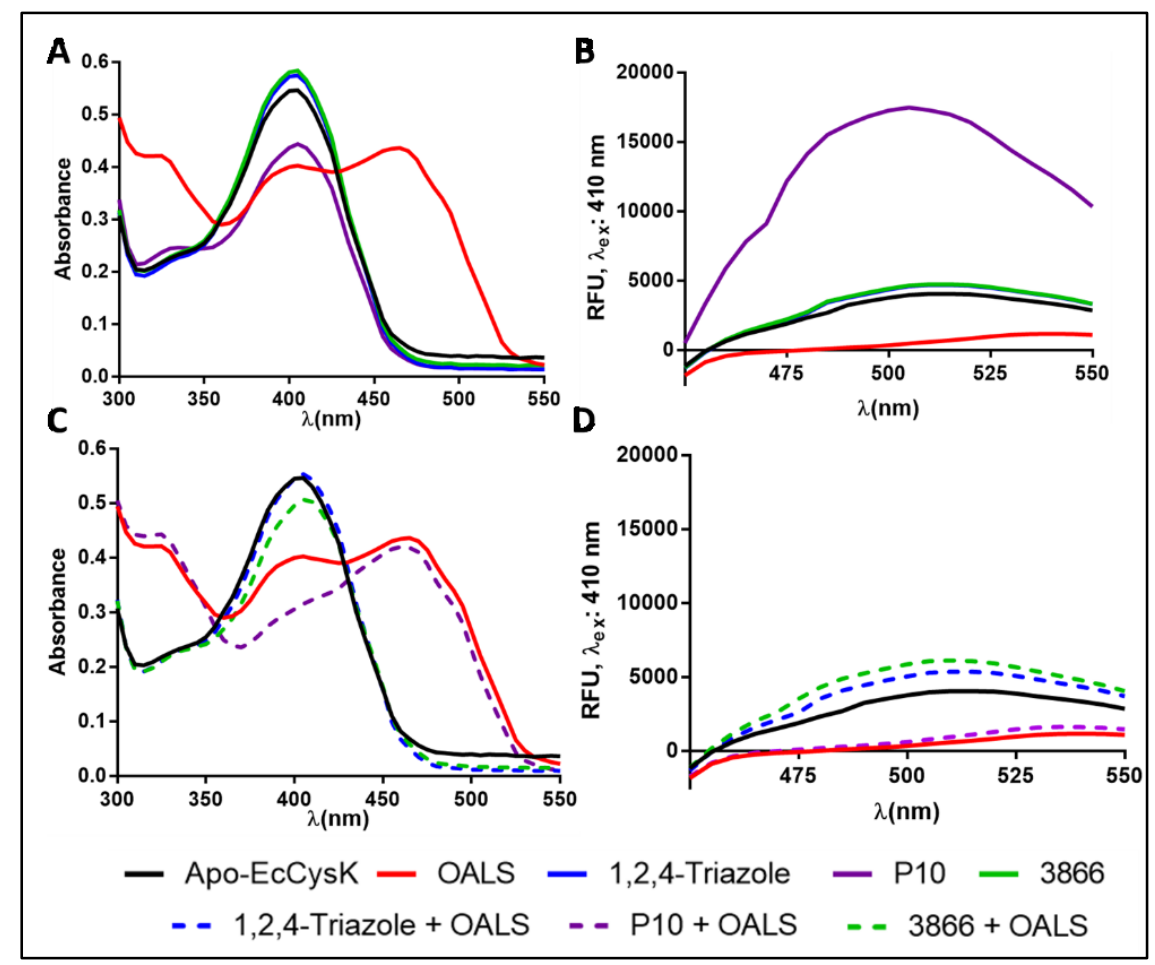

Figure 4-8. Spectroscopic profiling of EcCysK in combination with native substrate and CysK inhibitors.

The final concentration of the EcCysK enzyme is $100 \mu \mathrm{M}$ and all ligands were added at $500 \mu \mathrm{M}$. A. Scanning absorbance of EcCysK with individual ligands. B. Fluorescence spectrum of EcCysK with individual ligands. C. Absorbance spectrum of EcCysK with combinations of OALS substrate and inhibitors. D. Fluorescence spectrum of EcCysK with combinations of OALS substrate and inhibitors. 
observed in combination with the OALS substrate (Figure 4-8C, D). The combination of either 1,2,4-triazole or 3866 with OALS resulted in the restoration of both the absorbance and emission spectra to what was observed with the apoenzyme. Addition of EcP10 resulted in only subtle changes to either the absorbance or fluorescence spectra relative to the CysK-OALS complex.

The initial spectroscopic studies were carried out with single concentrations of enzyme, inhibitor, and substrate. Additional experiments revealed that the observed changes in the absorbance spectra exerted by both OALS binding and the 3866 interaction with the EcCysK-OALS complex are concentration-dependent (Figure 4-9). This dose dependency confirmed that the abrogation of PLP-OALS aminoacrylate formation imparted by 3866 is a true effect.

In contrast to EcP10, the spectroscopy studies yielded no evidence of observable direct binding effects between the triazole inhibitors and CysK in the absence of OALS. Furthermore, attempts at the thermal melt assay and surface plasmon resonance failed to show biophysical interactions between TAT molecules and CysK (data not shown). This implies that the effect of the TAT series is distinct from SAT C-terminal peptidomimetics which directly inhibit the CysK enzyme (Salsi 2010). The OALS substrate appears to need to be present for interaction of 3866 and 1,2,4-triazole with the enzyme (Salsi 2010). The absence of spectra indicating an external PLP linkage in the presence of both OALS and triazole inhibitor could indicate that a false product has been formed and released from CysK, which is the mechanism proposed to occur with 1,2,4-triazole (Kredich 1975).

\section{The transcriptional response of 3866-treated $E$. coli cells}

The biosynthetic pathways involved in cysteine biosynthesis, sulfur metabolism, and the closely related folate cycle and the methyl cycle are highly regulated by both the products and intermediate metabolites of these pathways. A likely hypothesis is that the effect of the TAT compounds on CysK will disrupt the cellular pools of associated metabolites, and therefore may alter normal regulation of the transcription of genes within these and potentially other metabolic pathways. Results from these studies will garner further mechanistic insights into the MOA of TATs.

Kredich and coauthors proposed that the growth inhibitory effect of 1,2,4-triazole on $S$. typhimurium was due to false product formation between OALS and the triazole. In addition to cysteine starvation, reduced intracellular pools of OALS, which induces sulfur-associated metabolic processes, resulted in downregulation of component enzymes in the RSAP (Kredich 1975). To study whether 3866 disrupted transcription in the RSAP or other closely associated metabolic pathways, an RNA-seq study was carried out with E. coli K12 preparations treated with 3866 . Two media conditions were used due to the high sensitivity of RSAP-associated gene transcription to the available sulfur source.

Inorganic sulfate is known to downregulate transcription of this pathway, which may mask potential effects of the triazole inhibitors on sulfur metabolism (Gyaneshwar 2005). 


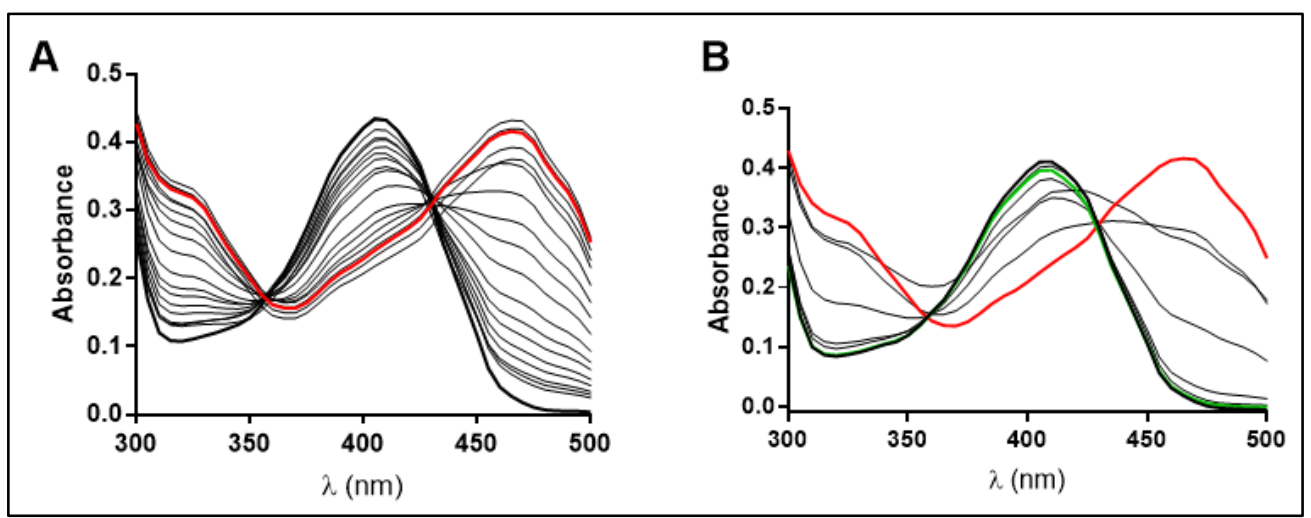

Figure 4-9. Dose-dependent spectroscopic effects.

Both OALS substrate and triazole inhibitor 3866 have dose dependent effects on the scanning absorbance profile of CysK. A. OALS gradient. Apo-CysK is represented by a bold black line. Bold red line represents highest concentration of OALS at $1 \mathrm{mM}$, and other black lines represent intermediate concentrations of OALS. B. Gradient of 3866 on OALS-CysK complex. Apo-CysK is again represented by bold black line, OALS-CysK complex with no inhibitor is represented by red line, bold green line represents 3866 and CysK without OALS, and all other black lines are intermediate concentrations of 3866 on the OALS-CysK complex. 
In addition to the standard M9 recipe (ATCC 2511) used for previous experiments, which has magnesium sulfate as a sulfur source, M9T media was used, which is the same media composition with the exception of taurine representing the sulfur source.

Before studying inhibitor effects, differences in the E. coli transcriptome between the two media were studied. Relative to M9, genes from the ssu and tau operons were the most upregulated in M9T (Figure 4-10). The proteins necessary for transport and sulfur acquisition from aliphatic sulfonates and taurine are encoded by the $s s u$ and tau operons, respectively. These effects are in agreement with the well-characterized homeostatic responses in E. coli to available sulfur sources (Gyaneshwar 2005). The ssu and tau operons are both negatively regulated by adenosine 5'-phosphosulfate (APS), which is the first RSAP intermediate after the transport of extracellular inorganic sulfate such as the magnesium sulfate that is present in M9. Both operons are also regulated by cellular concentrations of OALS, which may decrease upon CysK-mediated false product formation (Kredich 1975). Therefore, the two media conditions are suitable to study the potentially varied transcriptional effects associated with $E$. coli growth inhibition in either M9 or M9T.

The transcriptional effect of 3866 treatment of E. coli K12 in both M9 and M9T was examined. The altered genes were evaluated using both volcano plot representation (Figure 4-11) as well as through pathway analysis of significantly affected genes (Figure 4-12). Criteria for significant transcriptional changes were again defined as a $P$ value $\leq$ 0.01 and a two-fold change in gene transcription.

The most striking transcriptomic effect of 3866 was the marked downregulation of genes involved in sulfur metabolism in M9T (Figures 4-11A and 4-12A). Sulfur homeostatic responses are regulated by two major transcription factors, $\mathrm{CysB}$ and $\mathrm{cbl}$ (Gyaneshwar 2005). CysB, which is positively controlled by OALS, is the main regulator of sulfur homeostatic responses and is necessary for transcription of cbl. CysB regulates the cys, ssu, and tau operons encoding the RSAP, aliphatic sulfonate metabolism, and alkane sulfonate metabolism, respectively. The cbl transcription factor positively regulates the $s s u$ and tau operons and is negatively regulated by APS generated in the RSAP. The expression of cbl was downregulated in response to 3866 along with the $s s u$, tau, and cys operons. Since CysB expression was not significantly altered, a plausible explanation for the downregulation of these genes is a reduced cellular pool of the CysB co-activator OALS. This would agree with the CysK-mediated false product mechanism described with 1,2,4-triazole (Kredich 1975). Furthermore, transcriptional studies in S. typhimurium have shown a similar negative effect of 1,2,4-triazole on the cys operon (Turnbull 2008).

These major transcriptomic effects on sulfur metabolism were not observed in the standard M9 media, likely due to the negative effect of APS on gene transcription associated with sulfur homeostatic responses. However, dysregulation of genes in pathways linked to the biosynthesis of several amino acids, the shikimate pathway, and nucleotide biosynthesis was observed in both medias (Figure 4-12). The glycine cleavage system, which plays a key role in both serine and glycine catabolism and is 


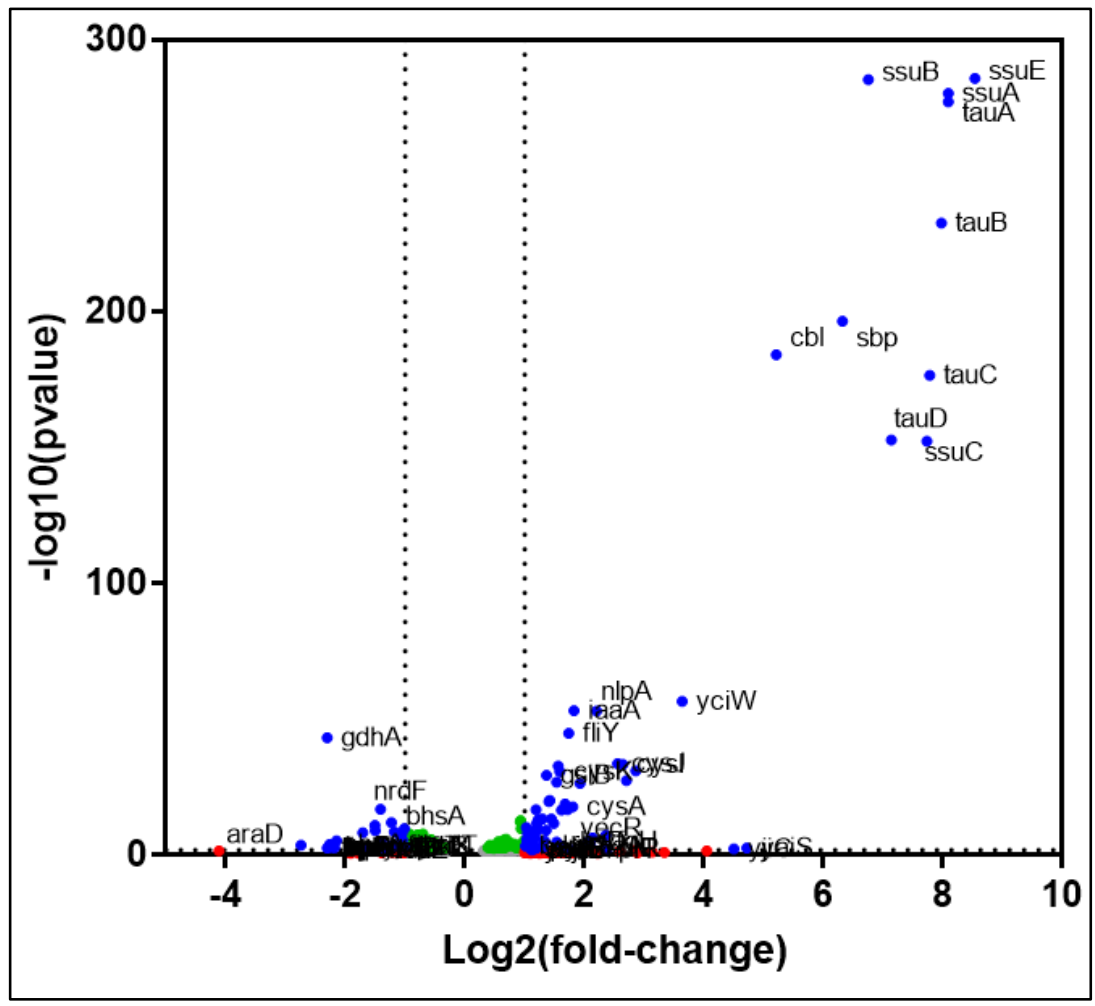

Figure 4-10. Volcano plot of global transcriptional changes in M9-based media with taurine as a sulfur source compared to magnesium sulfate as the sulfur source. Dashed lines represent cutoffs for significant fold-change of transcription at 2 and a Pvalue of 0.01 . Genes with significant change in expression and P-value are in blue, genes with significant transcriptional change but a P-value of $>0.01$ are in red. Green dots represent genes with insignificant changes in transcription in terms of both fold-change and P-value. Grey dots represent genes with a P-value $>0.01$ and no significant transcriptional change. 

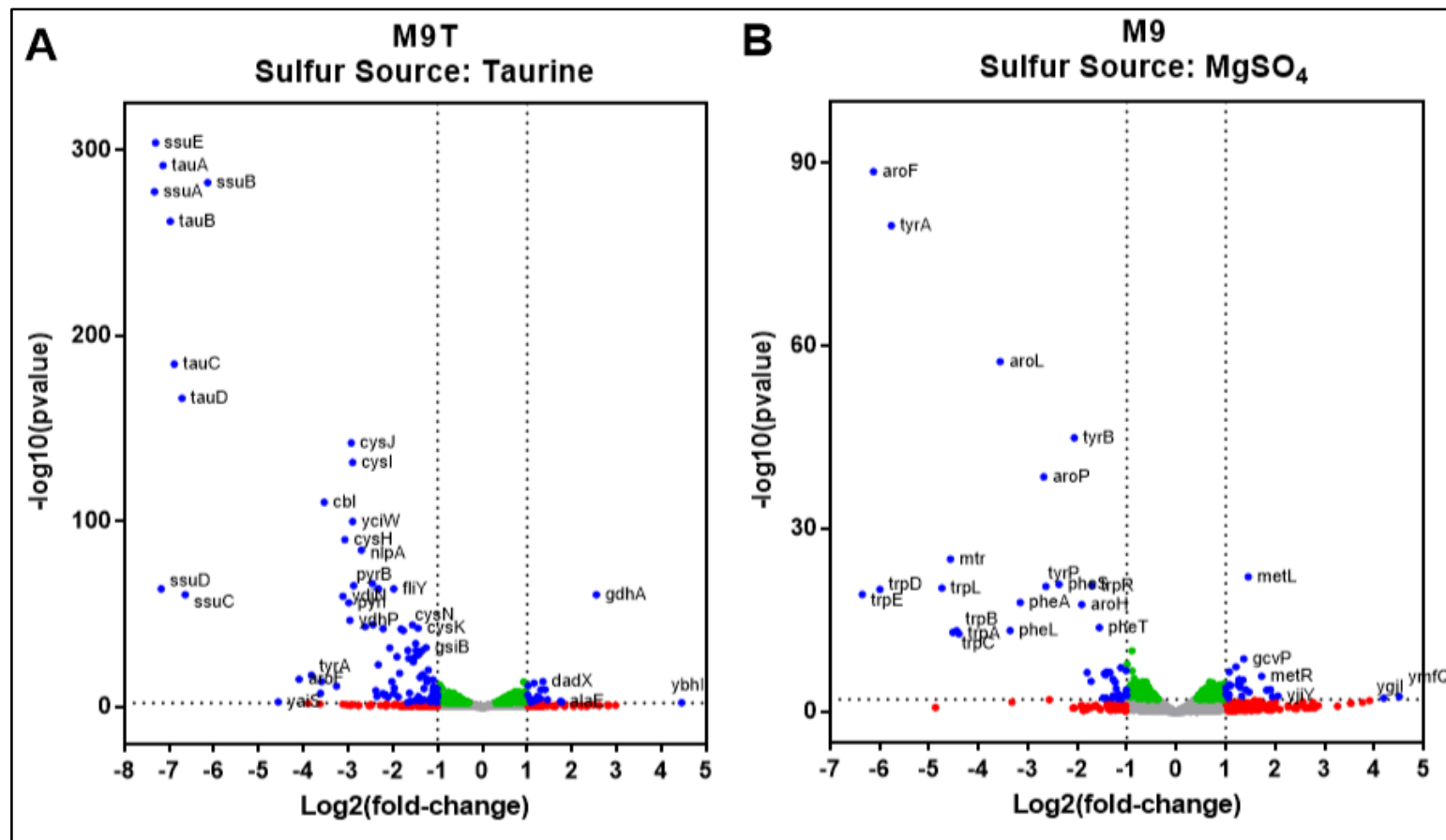

Figure 4-11. Volcano plots of transcriptional responses to TAT treatment. Dashed lines and coloring are similar to Figure 4-10. A. Global transcriptional response in standard M9 recipe (ATCC 2511) with taurine as the sulfur source. B. Global transcriptional response in M9 with magnesium sulfate as the sulfur source. 


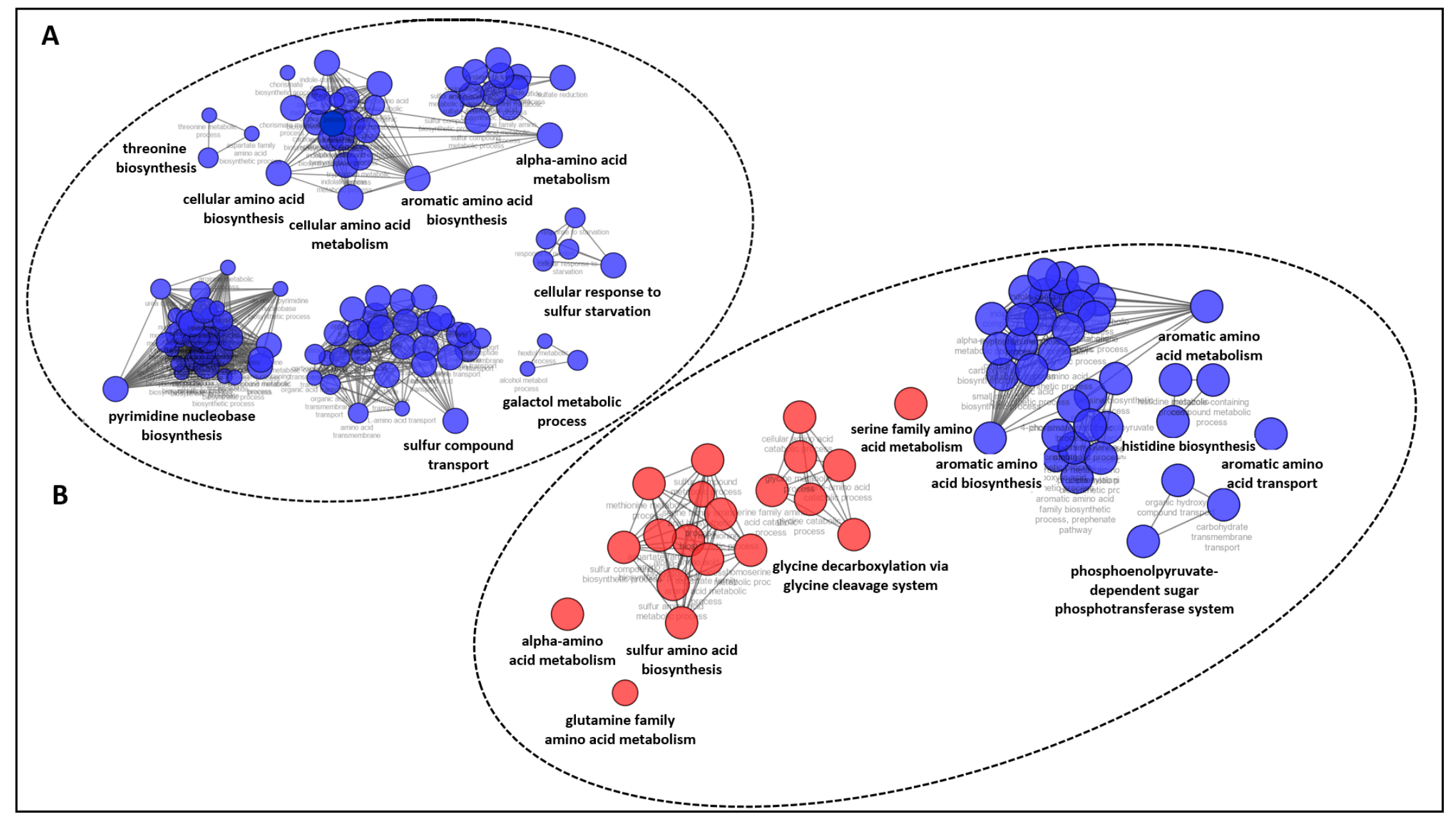

Figure 4-12. E. coli pathway networks transcriptionally altered by treatment with 3866 .

Genes with both a P-value $\leq 0.01$ and a $\log 2$ fold change of $\geq 1$ were selected for analysis. Blue and red shading represent downregulation and upregulation, respectively. A. Pathway analysis in M9T with taurine as the sulfur source. B. Pathway analysis in M9 with magnesium sulfate as the sulfur source. 
highly interconnected with the folate cycle, was upregulated in M9 in response to 3866 treatment.

Notably, in both conditions metabolic processes associated with aromatic amino acid biosynthesis and transport were substantially downregulated (Figures 4-11B and 412). The relationship of aromatic amino acid metabolic processes with cysteine biosynthesis is not as obvious as the sulfur-associated genes, however there are various biological associations between aromatic amino acid biosynthesis, sulfur metabolism, and folate biosynthesis. Chorismate is a shared precursor for both $p \mathrm{ABA}$ and aromatic amino acids (Dosselaere 2001). The transcription of the folA gene is under the control of TyrR, which also regulates aromatic amino acid metabolism (Yang 2007). Tryptophan and cysteine both share serine as a precursor. In combination with the tryptophan corepressor, TrpR negatively regulates the tryptophan biosynthetic operon as well as genes encoding various steps of the shikimate and chorismate pathways responsible for generation of aromatic amino acid precursors. The other two aromatic amino acids negatively regulate associated pathways, indicating a buildup of aromatic amino acids could be occurring in response to 3866 . Aromatic amino acid biosynthesis in plants is triggered in response to sulfur starvation, which is hypothesized to be a response to glutathione depletion to maintain redox homeostasis (Nikiforova 2006). Further experimentation is required to elucidate the exact relationship between aromatic amino acid biosynthesis and other aspects of cysteine, folate, and sulfur metabolism. However, these findings suggest the effects of inhibiting CysK may extend well beyond cysteine biosynthesis and dysregulate other metabolically significant pathways.

\section{Conclusions and Future Perspectives}

Altogether, the data presented in this study provide multiple indications that CysK is the target of the TAT inhibitors obtained in the HTS in Chapter 3. The phenotype of 3866 , which can be reversed by cysteine supplementation, is consistent with the phenotype observed with the cysteine auxotrophic $\Delta c y s K$ strain (Figure 4-5A). Nonsynonymous mutations in $c y s K$ were present in the spontaneous mutants (Table 4-3), and the introduction of the common mutation S70C in E. coli conferred resistance (Figure 4-5B). Metabolites closely linked to cysteine biosynthesis imparted substantial antagonism of the growth inhibitory effect of the triazoles (Table 4-6). The 3866 inhibitor also showed in-vitro interactions with the EcCysK-OALS complex through spectroscopy (Figures 4-8 and 4-9). These findings link the observed MIC activity with target inhibition in agreement with established postulates for target determination during the drug discovery process (Silver 2011, Copeland 2005).

An ongoing goal is to confirm the inhibition modality of the TATs against CysK. Some evidence of a false product mechanism has already been encountered. Various nitrogen-containing heterocycles are known to interact with CysK from bacteria and plants via a false-product mechanism (Kredich 1975, Ikegami 1988). The triazole portion of the TATs is essential for activity and may be the component of the molecule responsible for false product formation (Figure 4-6). Furthermore, all cell-based and spectroscopic studies including 1,2,4-triazole indicated this molecule interacts with $E$. 
coli and the CysK protein in a manner similar to the TAT series, and the interactions with the enzyme appear distinct from inhibitory peptides (Table 4-6 and Figure 4-8). The TATs demonstrated the expected transcriptomic effects on sulfur metabolic processes that are associated with 1,2,4-triazole treatment and OALS depletion (Kredich 1975, Turnbull 2008) (Figure 4-11B). The shutdown of these genes, which are under the control of the primary regulator $\mathrm{CysB}$, relies on the depletion of the CysB co-activator OALS, which would occur if a false product is formed between OALS and 3866. These data provide evidence toward a MOA through false product formation as described previously between CysK and 1,2,4-triazole (Kredich 1975).

Further studies are required to confirm a true cellular depletion of OALS as well as the formation of a false product, which was not directly addressed in this chapter. Bioanalytical studies are ongoing to investigate this putative false product reaction in both in vitro CysK-mediated reactions and in 3866-treated E. coli cells. Furthermore, efforts to optimize crystallization conditions for the recombinant $E c C y s K$ enzyme are also ongoing. Structural information on the molecular interactions of the TAT inhibitors obtained through X-ray crystallography may elucidate the inhibition modality and augment SAR efforts towards this inhibitor series.

Inhibition of metabolic targets that are unique to bacteria and central to fundamental metabolic processes is an exciting avenue for obtaining desperately needed new antibiotic targets. CysK is one such example of a potential new target that is absent in humans and situated at an important enzymatic branchpoint between sulfur assimilation, cysteine biosynthesis, and the methyl cycle. Similar to the antifolates, which have saved thousands of lives over nearly a century of use in humans, other antimetabolites could provide essential treatments desperately needed for multi-drug resistant pathogens. 


\section{CHAPTER 5. EXPLORING THE INTRACELLULAR TARGET ENGAGEMENT OF ANTIFOLATES IN GRAM-NEGATIVE BACTERIA}

\section{Introduction}

\section{Molecular methods to explore target engagement in Gram-negative cells}

The GN cell physiology presents several challenges to the drug discovery process. GN permeability, residence time, and efflux susceptibility all factor into the in vivo efficacy of the molecules of interest, and assessment of these qualities early in the drug development process is crucial (Silver 2016). A lack of early stage tools to measure whole cell uptake and target engagement are common roadblocks, especially for GN bacteria. Biological tools to assess and optimize GN potency early-on could improve the attrition rates associated with inhibitor series achieving in vivo and even clinical efficacy.

In response to the formidable worldwide issue of AMR GN infections, much effort has been put forth in recent years towards improving techniques to determine compound accumulation in GN bacteria. Liquid chromatography-mass spectrometry (LC-MS) approaches have been optimized to track the accumulation of compounds into the GN cell (Davis 2014, Zhou 2015). A recent compound accumulation study on 180 diverse molecules indicated that the presence of a primary amine, low globularity, amphiphilicity, and low rigidity all contribute to improved GN accumulation (Richter 2017). Raman spectroscopy has also proven useful for label-free tracking of GN compound accumulation (Heidari-Torkabadi 2015).

Although LC-MS and Raman spectroscopy are validated methods to study GN compound accumulation, they do not show direct indications of target engagement. An emerging technique employed in mammalian systems is the cellular thermal shift assay (CETSA), which can simultaneously show evidence of cell entry and target engagement in the intact cell system (Martinez 2013, Jafari 2014). This technique relies on the basic principles of the in vitro thermal shift assay, wherein ligand binding often leads to thermostabilization of the protein target (Waldron 2003). CETSA allows for the study of this phenomenon in intact cells and prepared lysates, allowing for target interaction to be studied in a more physiologically relevant context.

To perform CETSA, a cell or lysate preparation originating from the species of interest is treated with the desired drugs. For cell preparations, a wash step is often included after drugging to ensure compound that did not penetrate the cells does not interact with the target during subsequent lysis steps. After drugging, the samples are subjected to gradient heating, after which a method of determining the drug-induced heatstabilization of the target is employed. A variety of detection methods have been developed in recent years. The standard method involves removal of aggregated proteins from the samples, followed by immunodetection of the resulting soluble fractions (Martinez 2013). 
CETSA takes into account several important biological factors that many in vitro assays do not including the ability to permeate the cell membrane of the species targeted, trapping in cellular compartments, modification of the drug in the cell, and the activity in the physiologically relevant environment of the cytoplasm (Martinez 2013). Since the methods required to carry out CETSA are amenable to bacterial systems, this technique could be useful for studying compound entry in GN cells.

\section{Next-generation inhibitor development of antifolates}

The FBP is a promising target for the development of next-generation antibiotics, and recent insights into the structure and function of component enzymes have allowed for the rational design of next-generation therapeutics (Bermingham 2002). For example, propargyl-linked antifolates (PLAs) are designed to have high affinity towards resistant forms of the DHFR enzyme, and structure/function studies have guided the optimization of this series (Lombardo 2016). These compounds extend further into a hydrophobic pocket in the DHFR active site than TMP, while gaining additional hydrophobic interactions through the propargyl linker. This increased engagement may explain the affinity maintained between different forms of the enzyme and potent MICs observed against TMP-resistant strains. Various PLA inhibitor series have been generated with efficacy against both GP and GN pathogens (Reeve 2016, Schocchera 2016).

Similar to the PLAs, the pyridazine series targeting DHPS has also been optimized using an improved understanding of folate biosynthesis machinery in recent years. These compounds engage the pterin binding site, which is well conserved and predicted to be less resistance prone than the flexible loop region where sulfonamides bind (Lever 1985, Zhao 2012). An unfortunate discrepancy in this series is a lack of detectable MIC activity despite a high enzyme affinity. This could be due to either poor bacterial permeation or differences in the molecular mechanism pyridazines exert against DHPS as compared to sulfonamides.

\section{Major efflux systems in Gram-negative bacteria}

Bacteria have evolved efflux mechanisms to efficiently shuttle a variety of molecules out of the periplasm and cytoplasm. This results in both intrinsic and acquired resistance among pathogens. Resistance-nodulation-division (RND) efflux pumps are widespread among several GN species and are often responsible for MDR (Nikaido 2009). To understand the mechanistic basis of efflux, the major efflux machinery of several GN species has been characterized. RND pumps have three components including a transporter extending from the cytoplasmic membrane to the periplasm, an outer-membrane channel, and a membrane fusion protein (Silver 2016). The bestcharacterized RND efflux complex is in E. coli, consisting of the TolC outer membrane channel, the AcrB inner membrane transporter, and the AcrA fusion protein. E. coli strains with the $t o l C$ gene knocked out lose their intrinsic resistance to a variety of antibiotic classes, indicated by substantial decreases in MIC values (Tamae 2008). 
Comparison of MICs between tolC knockout strains and parent strains are routinely used to assess efflux susceptibility during antimicrobial testing.

\section{Improved strategies to develop GN-effective antifolates}

Within this chapter, CETSA techniques are adapted to the GN cell system to study intracellular target engagement and efflux susceptibility of next-generation antifolates. A panel of E. coli strains is assembled that enables the study of efflux susceptibility and the effect of target overexpression on the growth inhibitory effects of antifolates. Episomal expression of FLAG-tagged DHPS and DHFR enables the CETSA technique by allowing for immunodetection of target proteins. Ligand-induced thermostabilization is measured after treatment of both prepared lysates and intact cells to compare engagement with targets in the physiological cytoplasmic environment as well as in the presence of the intact GN cell membrane system. Important information is gleaned on the ability of DHPS inhibitors to engage the target within the GN cell as well as efflux susceptibility of select PLAs. For the pyridazine showing the best intracellular target engagement through CETSA, effects of this inhibitor on the E. coli transcriptome are observed.

\section{Materials and Methods}

\section{Minimal inhibitory concentration determination}

MIC testing was performed as previously described in Chapter 2 and was carried out in both LB and M9.

\section{Surface plasmon resonance}

Poly-His tagged BaDHPS protein was kindly gifted from the laboratory of Dr. Stephen White. SPR experiments were performed by the Molecular Interaction Analysis core at St. Jude Children's Research Hospital. SPR experiments were conducted at $20^{\circ} \mathrm{C}$ using a ForteBio Pioneer FE optical biosensor. BaDHPS was immobilized on polycarboxylate hydrogel-coated gold chips preimmobilized with nitrilotriacetic acid (HisCap chips; ForteBio). The chip was primed in $10 \mathrm{mM}$ HEPES pH 7.4, $150 \mathrm{mM}$ $\mathrm{NaCl}, 50 \mu \mathrm{M}$ EDTA, $0.005 \%$ Tween 20 (chelating buffer) and was preconditioned at 10 $\mu \mathrm{L} / \mathrm{min}$ with three 60 second injections of wash buffer $(10 \mathrm{mM}$ HEPES pH 8.3, $150 \mathrm{mM}$ $\mathrm{NaCl}, 350 \mathrm{mM}$ EDTA, $0.05 \%$ Tween 20 ) and one 60 second injection of chelating buffer before being charged with a 60 second injection of $500 \mu \mathrm{M} \mathrm{NiCl}_{2}$ in chelating buffer. After priming into the binding buffer (20 mM Tris $\mathrm{pH} 7.6,150 \mathrm{mM} \mathrm{NaCl}, 1 \mathrm{mM}$ TCEP, $0.01 \%$ Tween $20,5 \%$ DMSO), BaDHPS was injected until $\sim 3600$ RU of protein were captured. One flow cell on the chip was charged with $\mathrm{Ni}^{2+}$ without adding protein to be used as a reference cell. 
Compounds were prepared in binding buffer at two concentrations (50 and 100 $\mu \mathrm{M}$ for SMX; 25 and $50 \mu \mathrm{M}$ for $568 ; 2.5$ and $5 \mu \mathrm{M}$ for $1462,3893,3894,852$; and 100 and $200 \mathrm{nM}$ for 1532 and a control DHPS inhibitor 9229). The solutions were injected in duplicate for each concentration at a flow rate of $100 \mu \mathrm{L} / \mathrm{min}$ using the OneStep Injection feature, which exploits Taylor dispersion to generate a concentration gradient that provides a full titration of analyte in a single injection (Quinn 2012a, Quinn 2012b). A series of buffer-only (blank) injections were included throughout the experiment to account for instrumental noise. All compounds fully dissociated from the protein surfaces, eliminating the need for a regeneration step. The data were processed, doublereferenced, solvent corrected and analyzed using the software package Qdat (version 4.3.1.2, ForteBio). Kinetic rate constants and affinities were determined by fitting the data to a 1:1 interaction model.

\section{Whole cell CETSA}

CETSA methods for GN bacteria were adapted from a protocol for CETSA on mammalian cell preparations (Jafari 2014). A single colony of the strain to be tested was grown overnight in LB with $100 \mu \mathrm{g} / \mathrm{mL}$ carbenicillin. The overnight culture was subbed 1:100 in LB with no selective agent and grown to a mid-log $\mathrm{OD}_{600}$ between 0.4 and 0.6 . Mid-log bacteria was drugged at a final concentration of $100 \mu \mathrm{M}$ for the DHPS inhibitors and $10 \mu \mathrm{M}$ for the DHFR inhibitors, with a final DMSO concentration of $2 \%$. The drugged cultures were allowed to shake at $225 \mathrm{RPM}, 37^{\circ} \mathrm{C}$ for 30 minutes, after which the cells were moved to ice and $300 \mathrm{uL}$ taken from each culture for an $\mathrm{OD}_{600}$ read. The remaining culture was spun at $4,000 \times \mathrm{G}$ for 5 minutes. The supernatant was discarded and each pellet washed with $2 \mathrm{~mL}$ CETSA buffer, which consisted of $20 \mathrm{mM}$ Tris, $\mathrm{pH}$ $7.5,200 \mathrm{mM} \mathrm{NaCl}$ with one protease inhibitor (cOmplete mini 11836170001 ) per $10 \mathrm{~mL}$ of buffer. The cultures were spun as before and supernatant discarded. During the centrifugation and wash steps, $\mathrm{OD}_{600}$ values were determined and the resuspension volume calculated. For DHPS, pellets were suspended at $4 \mathrm{X}$ the $\mathrm{OD}_{600}$. For DHFR, pellets were resuspended at $2 \mathrm{X}$ the $\mathrm{OD}_{600} .30-100 \mu \mathrm{L}$ was aliquoted into PCR strips, and the cells were administered an optimized heat gradient using the Veriti Thermal Cycler for 3 minutes, followed by a 3-minute rest at room temperature. The cells were then flash-frozen in liquid Nitrogen. The cells were thawed and lysed by adding $5 \mathrm{uL} 10$ $\mathrm{mg} / \mathrm{mL}$ lysozyme for every $100 \mathrm{uL}$ cells and incubated at $37^{\circ} \mathrm{C}$ for 30 minutes. The lysed cells were pelleted at $20,000 \mathrm{xG}$ for 20 minutes at $4^{\circ} \mathrm{C} .19 .5 \mu \mathrm{L}$ supernatant was collected from each sample and mixed with $7.5 \mu \mathrm{L}$ LDS in a 96 well PCR plate. Plates were stored at $-20^{\circ} \mathrm{C}$ for up to two weeks before immunodetection.

\section{Lysate CETSA}

The cells used for the lysate CETSA strains were grown as described for the whole cell CETSA to a mid-log OD 600 . The cells were spun and washed in CETSA buffer as before and resuspended at the CETSA $\mathrm{OD}_{600}\left(4 \mathrm{X} \mathrm{OD}_{600}\right.$ for DHPS and $2 \mathrm{X}$ $\mathrm{OD}_{600}$ for DHFR). The cells were then flash-frozen in $1 \mathrm{~mL}$ aliquots and stored at $-80^{\circ} \mathrm{C}$ 
until ready to use. The cells were lysed as previously described for the whole cell CETSA followed by spinning at $20,000 \times \mathrm{G}, 20$ minutes at $4^{\circ} \mathrm{C}$. The supernatants were retained and pooled if multiple tubes were lysed. Drug tubes were prepared by adding 4 $\mu \mathrm{L}$ of either DMSO or $500 \mu \mathrm{M}$ drug to $1.5 \mathrm{~mL}$ tubes. $196 \mu \mathrm{L}$ lysate was added to each

drug tube so that the final drug concentration was $100 \mu \mathrm{M}$ for the DHPS inhibitors or 10 $\mu \mathrm{M}$ for the DHFR inhibitors. The tubes were briefly vortexed then taped to the floor of an incubator shaker and allowed to equilibrate for 30 minutes at $37^{\circ} \mathrm{C}, 225 \mathrm{RPM}$. After drugging, the lysate was aliquoted into PCR strips as before. All subsequent methods including CETSA gradient heating and subsequent processing were performed as described for the whole cell CETSA, except no flash-freeze or lysis step was included.

\section{Lysate ITDRFCETSA}

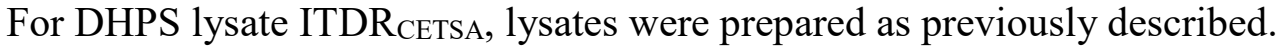
Drugs were diluted 1:5 starting at $5 \mathrm{mM}$, then $1 \mathrm{uL}$ drug added to each of 5 tubes. Only DMSO was added to the remaining tube. $49 \mu \mathrm{L}$ lysate was added to each tube and allowed to incubate as before. During the CETSA heating step, all tubes were heated at a single temperature of $58^{\circ} \mathrm{C}$. All subsequent processing followed the methods for the lysate CETSA.

\section{Western blotting}

$1.5 \mu \mathrm{L}$ of 10X DTT was added to each $13.5 \mu \mathrm{L}$ CETSA sample/LDS mixture and heated at $70^{\circ} \mathrm{C}$ for 10 minutes. $10 \mu \mathrm{L}$ of each sample was loaded onto a NuPage $12 \%$ Bis-Tris $1 \mathrm{~mm} 15$ well gel. $20 \mathrm{ng}$ FLAG-BAP protein (Sigma P7582) and a blank sample including only LDS and CETSA buffer were included as controls. The gel running buffer was 1X MES SDS running buffer. The gels were run at $200 \mathrm{~V}$ for 1 hour. Afterwards the gels were transferred using the iBlot 2 system to transfer to a nitrocellulose membrane. The membranes were each blocked in $5 \mathrm{~mL}$ Odyssey TBS blocking buffer with $10 \%$ goat serum for one hour. Two $\mu \mathrm{L}$ of $\alpha$ FLAG primary antibody (abcam213519) was spiked into the block buffer and incubated overnight at $4^{\circ} \mathrm{C}$. The blots were washed three times for 5, 10, and 15 minutes in TBST, then incubated with secondary antibody (LI COR IRDYE 800CW 926-32219) at a 1:5000 dilution for one hour at room temperature. The blots were washed in TBST as before and imaged on an Odyssey CLx Imaging System.

\section{Band quantification and data analysis}

Image Studio Lite Ver 5.2 was used to quantify band density. For standard CETSA, the first band at the lowest temperature in the heat gradient was set as $100 \%$ relative band intensity (RBI) and the blank control was set as $0 \%$ RBI. The RBI of each band was calculated relative to those controls. The temperature gradient and associated RBI values were plotted in GraphPad Prism. For the ITDR CETSA values, the 
Boltzmann equation was used to calculate V50 and $\mathrm{R}^{2}$ values. Constraints were set when necessary to accommodate curve fitting. When replicate values were plotted, the SEM was represented on the aggregation curves with error bars.

\section{RNA-seq}

The RNA-seq study and data analysis, with the exception of the pathway analysis, was performed as described in Chapter 5 using the E. coli K12 MG1655 strain. Mid-log bacterial preparations were drugged at final concentrations of $146 \mu \mathrm{M}$ for 568 and 100 $\mu \mathrm{M}$ for 3893. To compare shared significant transcriptional changes between groups that were significantly altered, a Venn diagram was generated using Venny (Oliveros 2015).

\section{Results and Discussion}

\section{Selection of molecules to test in the GN CETSA model}

Various experimental antifolates show minimal or no activity in GN bacterial assays. Whether this is due to issues with GN permeability and efflux susceptibility has yet to be determined, so the fate of these molecules in terms of GN entry and intracellular target engagement was explored by optimizing CETSA in the GN model pathogen $E$. coli BW25113. Ten molecules were chosen for this study (Figure 5-1). Seven of these compounds target DHPS, including an SMX sulfonamide control and 568, which is the pterin-sulfamethoxazole inhibitor (Zhao 2016). The other five molecules are pyridazine inhibitors. Three of these inhibitors, 852, 1532, and 1462, are previously established pyridazines (Lever 1985, Zhao 2012). Two more recently synthesized inhibitors are 3893 and 3894. 3894 has the primary amine predicted to aid in GN entry, and 3893 is its synthetic precursor (Richter 2017). Although designed to have high affinity for the DHPS enzyme, none of the five pyridazines have reported biological activity for unknown reasons.

Additionally, three compounds were chosen to study DHFR in the GN CETSA model (Figure 5-1). TMP is an established DHFR inhibitor, serving as a control. UCP1195 and UCP1218 were kindly gifted from the lab of Dr. Dennis Wright at the University of Connecticut. These compounds are PLAs designed to have a high affinity for resistant forms of the DHFR enzyme (Lombardo 2016). Varying susceptibility to efflux was anticipated against the two PLAs, so they were chosen to study in the CETSA model to see if efflux could be measured. 


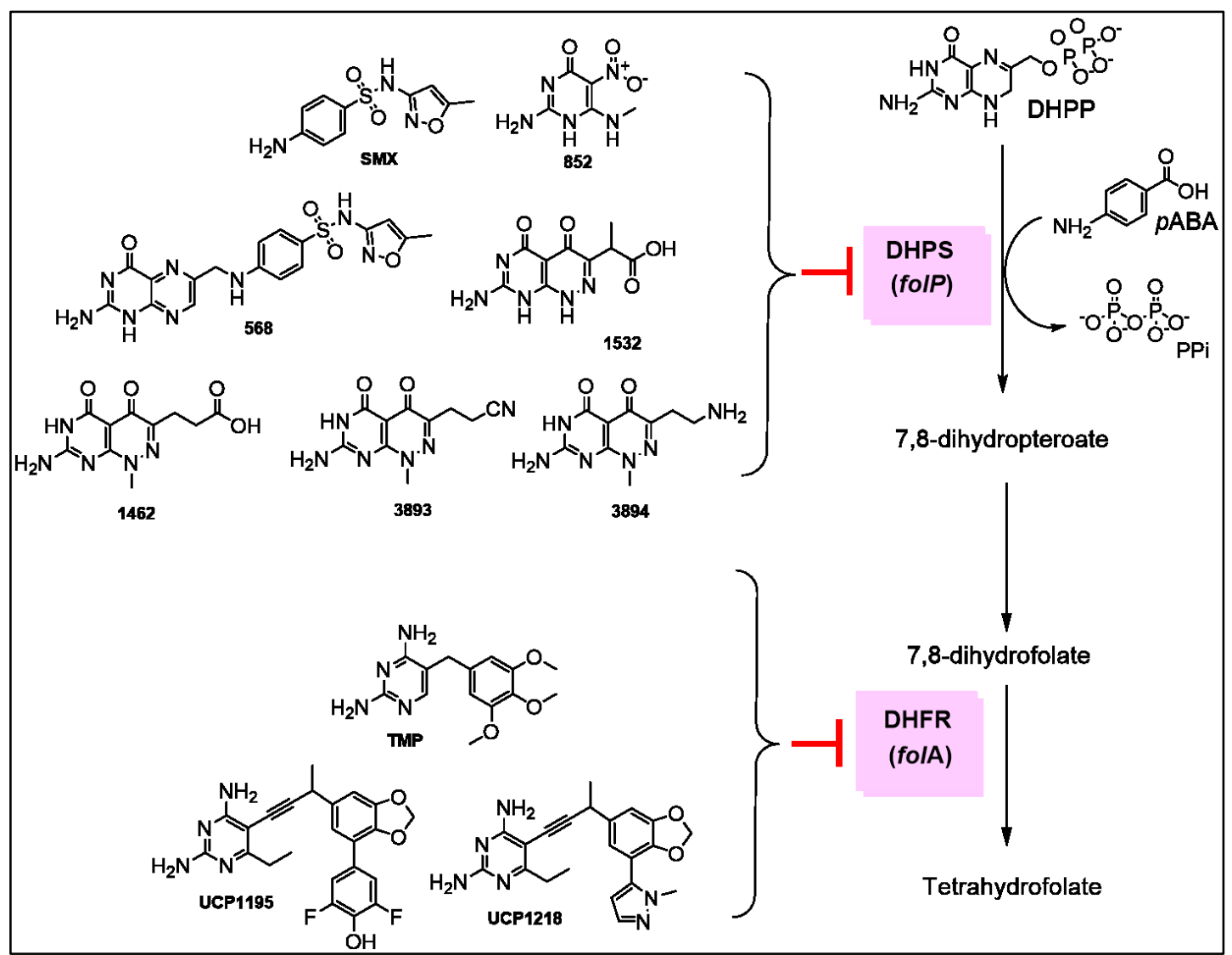

Figure 5-1. Established and experimental antifolates selected for the CETSA study.

The portion of the FBP including the DHPS and DHFR targets is shown. 


\section{Binding kinetics analysis of DHPS compounds using surface plasmon resonance}

Surface plasmon resonance (SPR) has proven to be a reliable in vitro method for tracking the binding affinity of DHPS inhibitors (Zhao 2012, Hammoudeh 2014). Within the set of DHPS-targeted molecules chosen for this study, only 1532 had been previously characterized using this approach (Zhao 2012, Compound 19). SPR was therefore performed on all selected DHPS compounds in order to compare in vitro target affinities with the CETSA data.

SPR was carried out with the $B$. anthracis enzyme. The DHPS enzyme from this species has shown favorable stability, reliability, and consistency with SPR testing (Zhao 2012). 1532 continued to have $\mathrm{nM}$ binding affinity represented by a KD of $17.1 \mathrm{nM}$ and had the highest affinity and slowest dissociation rate of the entire set (Table 5-1 and Figure 5-2). 852, 1462, and 3893 all had binding affinity within the nM range, while 568 and 3894 were in the low $\mu \mathrm{M}$ range. SMX did not exhibit any evidence of a binding profile in this experiment (Table 5-1 and Figure 5-2). Although sulfonamides interact with DHPS as part of their MOA, this mechanism involves binding within a flexible loop region to form a false product in the presence of DHPP. Interaction of DHPS with SMX when the pterin and PPi sites are unoccupied may not result in a strong enough conformational change to detect this biomolecular interaction using SPR technology. In contrast to SMX, all other compounds tested bind the pterin binding pocket and were designed to have strong binding interactions with multiple conserved residues in this site.

\section{Generation of $E$. coli strains constitutively expressing DHFR and DHPS}

The GN representative bacteria $E$. coli BW25113 was chosen for the GN CETSA study (Table 5-2). This strain is well characterized and is also the parent strain for the Keio collection, which allows for comparison with the available knockout strains (Baba 2006). For overexpression and immunodetection of DHFR and DHPS, the pPJ131 constitutive expression vector was kindly gifted from the laboratory of Dr. Charles Rock (Yao 2016). The folA and folP genes encoding DHFR and DHPS, respectively, were individually cloned into the plasmid with an N-terminal FLAG tag and a flexible glycine linker between the tag and the gene. These N-terminal modifications to the expressed proteins were designed to enable immunodetection of the expressed proteins via western blotting during CETSA. The plasmids were electrotransformed into both the parent strain and a tolC knockout strain derived from the Keio knockout strain JW5503 with the Kanamycin resistance marker removed ( $\Delta$ tolC Kan-S) (Table 5-2). Both sequencing and the ability to grow in the presence of the carbenicillin selective agent for pPJ131 confirmed the presence and correct sequence of the plasmids within each strain. 
Table 5-1. $\quad B a$ DHPS SPR data for DHPS-targeted compounds.

\begin{tabular}{ccccc}
\hline Compound & $\mathbf{k}_{\mathbf{a}}\left(\mathbf{M}^{-1} \mathbf{s}^{-1}\right)$ & $\mathbf{k}_{\mathbf{d}}\left(\mathbf{s}^{-1}\right)$ & $\mathbf{K D}(\mathbf{n M})$ & $\mathbf{R m a x}(\mathbf{R U})$ \\
\hline SMX & $\mathrm{ND}$ & $\mathrm{ND}$ & $\mathrm{ND}$ & $\mathrm{ND}$ \\
568 & $5.84( \pm 0.02) \times 10^{4}$ & $1.90( \pm 0.01) \times 10^{-1}$ & $3250( \pm 10)$ & $44.2( \pm 0.1)$ \\
852 & $5.13( \pm 0.01) \times 10^{5}$ & $4.32( \pm 0.01) \times 10^{-1}$ & $841( \pm 1)$ & $19.3( \pm 0.1)$ \\
1462 & $7.82( \pm 0.01) \times 10^{5}$ & $1.87( \pm 0.01) \times 10^{-1}$ & $239( \pm 1)$ & $21.5( \pm 0.1)$ \\
1532 & $7.44( \pm 0.02) \times 10^{5}$ & $1.27( \pm 0.01) \times 10^{-2}$ & $17.1( \pm 0.1)$ & $18.2( \pm 0.1)$ \\
3893 & $3.48( \pm 0.01) \times 10^{5}$ & $2.66( \pm 0.01) \times 10^{-1}$ & $763( \pm 2)$ & $27.4( \pm 0.1)$ \\
3894 & $1.69( \pm 0.01) \times 10^{5}$ & $4.24( \pm 0.01) \times 10^{-1}$ & $2500( \pm 10)$ & $33.5( \pm 0.1)$ \\
\hline
\end{tabular}

Note: $\mathrm{ND}=$ not detected. 


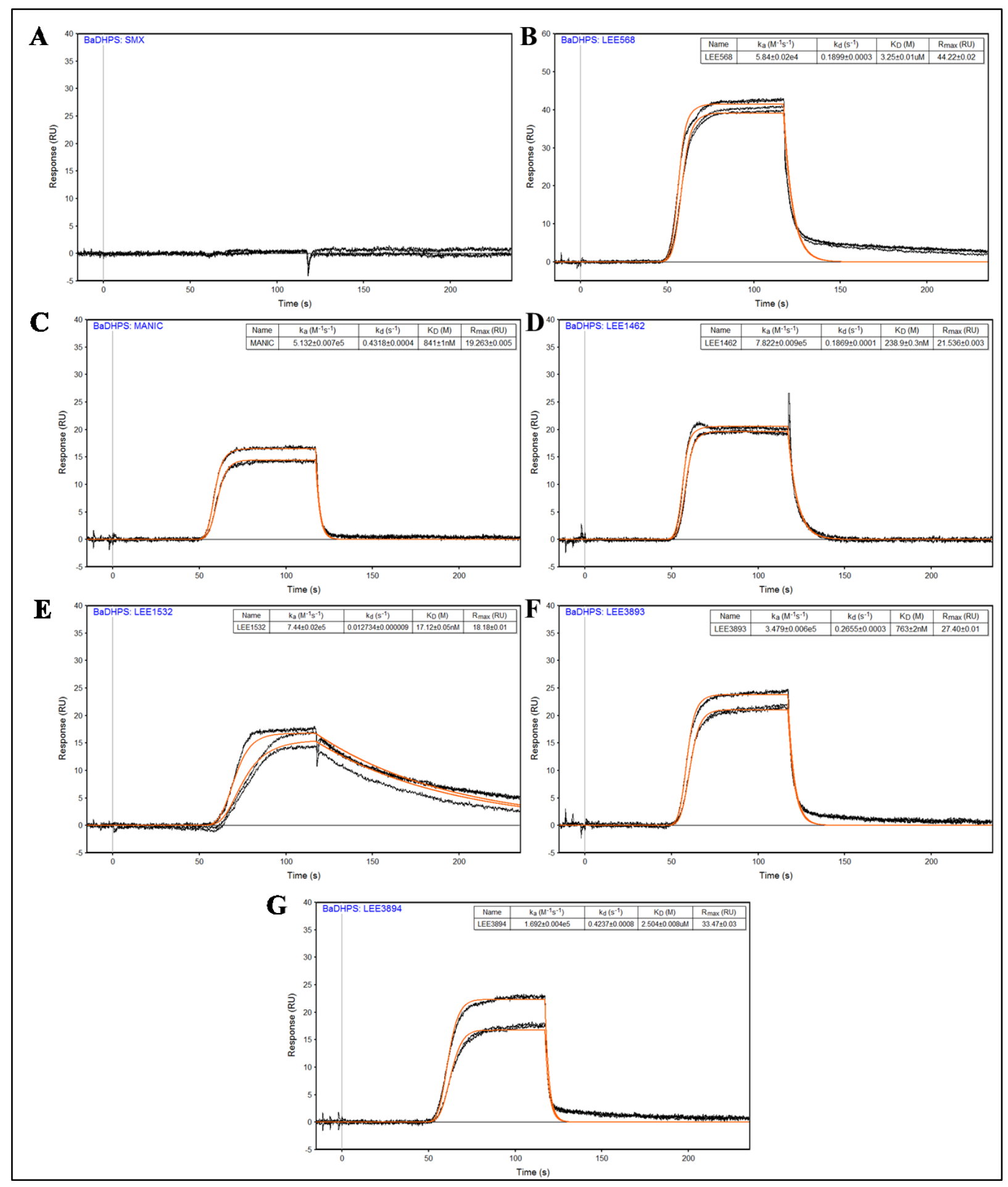

Figure 5-2. SPR sensograms for the DHPS-targeted molecules.

Sensograms are shown for A. SMX, B. 568, C. 852, D. 1462, E. 1532, F. 3893, G. 3894. 
Table 5-2. Strains used in the GN CETSA study.

\begin{tabular}{lll}
\hline E. coli Strain Background & \multicolumn{1}{c}{ Characteristic/Genotype } & \multicolumn{1}{c}{ Source } \\
\hline BW25113 & None (WT) & 2006 Baba \\
BW25113 & pPJ131 empty plasmid, CBN & This study \\
BW2513 & pPJ131:FLAG:folA, CBN ${ }^{\mathrm{R}}$ & This study \\
BW25113 & pPJ131:FLAG:folP, CBN & This study \\
$\Delta t o l C$ Kan-S & $\Delta t o l C$ & CGSC 14206 \\
$\Delta t o l C$ Kan-S:pPJ131 & $\Delta t o l C$, pPJ131 empty plasmid, CBN & This study \\
$\Delta t o l$ C Kan-S:folA & $\Delta t o l C$, pPJ131:folA, CBN ${ }^{\mathrm{R}}$ & This study \\
$\Delta t o l C$ Kan-S:folP & $\Delta t o l C$, pPJ131:folP, CBN & This study \\
\hline
\end{tabular}

$\mathrm{CBN}^{\mathrm{R}}=$ Carbenicillin resistant; Kan-S = Kanamycin sensitive; $\mathrm{CGSC}=$ Coli Genetic Stock Center. 


\section{Susceptibility profile of the $E$. coli CETSA panel against established and experimental antifolates}

In E. coli, overexpression of folA has been shown to confer resistance to TMP, while overexpression of folP does not affect sulfonamide susceptibility. This is due to the distinct MOAs of the two enzymes (Palmer 2014). TMP directly inhibits DHFR, and a higher cellular abundance of the target allows more enzyme to be available to perform the normal metabolic function of reducing dihydrofolate. On the other hand, sulfonamides have a false product mechanism wherein the inhibitor competes with $p \mathrm{ABA}$ to bind at the same site and form a false product with DHPP.

To examine whether overexpression of each enzyme exhibits similar effects and also determine the sensitivity of each compound to efflux, MICs were determined for the ten molecules chosen for the GN CETSA study (Figure 5-1). The MIC assays were carried out in both LB and M9 broth, since CETSA studies would be carried out in LB while the minimal nutrient environment of M9 prevents the interference of certain metabolites with detection of growth inhibition (Zlitni 2013). Among the control antibiotics, all strains harboring the pPJ131 plasmid were resistant to the carbenicillin selective agent (Tables 5-3 and 5-4). As expected, the $\Delta$ tolC Kan-S strains were hypersusceptible to the novobiocin control compound and showed more moderate susceptibility increases towards TMP and ciprofloxacin. The SMX MIC was not altered by overexpression of the folP gene, but overexpression of folA increased the MIC of all DHFR-targeted compounds in agreement with other studies (Palmer 2014).

Among the experimental DHPS compounds, 568 was the only potent compound against $E$. coli (Table 5-3). The only other DHPS-targeted compound with a detectable MIC was 852, which had a MIC at the highest concentration tested of $200 \mu \mathrm{M}$ in the $\Delta$ tolC Kan-S strain in M9. The DHFR-targeted inhibitors all appeared susceptible to efflux, especially the PLAs. This susceptibility testing confirmed the experimental antifolates are interacting with their target enzymes similarly to established antifolates. Efflux susceptibility was also confirmed for the two PLAs. The lack of MIC seen with the pyridazines, which effectively bind DHPS in the SPR assay, indicates issues with cell permeability and accumulation in the GN cell are possible barriers to growth inhibition.

\section{Optimization of western blotting conditions for CETSA panel}

The available commercial antibodies for many proteins in bacteria are scarce in comparison to what is available for mammalian targets. Antibody generation towards new targets can be laborious, time-consuming, and expensive. Therefore, the proteins in this study were episomally expressed in E. coli with a FLAG-tag, allowing for immunodetection with commercially available $\alpha$ FLAG antibodies (Table 5-2). Additionally, this allowed the same antibody set to be used for immunodetection of both targets, reducing variability introduced by using two distinct primary antibodies. The

plasmids were electrotransformed into both $E$. coli BW25113 and E. coli $\Delta$ tolC Kan-S (JW5503-KanS) to allow for CETSA testing in both efflux-intact and efflux-deficient 
Table 5-3. MICsos of $E$. coli CETSA panel in M9 minimal media $(\mu M)$.

\begin{tabular}{|c|c|c|c|c|c|c|c|c|c|c|c|c|c|}
\hline \multirow[b]{2}{*}{ E. coli Strain $^{\mathrm{a}}$} & \multicolumn{3}{|c|}{ Controls } & \multicolumn{7}{|c|}{ DHPS targeting } & \multicolumn{3}{|c|}{ DHFR targeting } \\
\hline & CBN & CPR & NVB & SMX & 1532 & 568 & 1462 & 3893 & 3894 & 852 & TMP & UCP1195 & UCP1218 \\
\hline BW25113 & 5.3 & 0.024 & $>200$ & 0.52 & $>200$ & 1.0 & $>200$ & $>200$ & $>200$ & $>200$ & 1.3 & 4.2 & $>50$ \\
\hline BW25113:pPJ131 & $>200$ & 0.012 & $>200$ & 0.68 & NT & 1.6 & NT & NT & NT & NT & 1.0 & 3.1 & $>50$ \\
\hline$\Delta$ tolC Kan-S & 1.6 & 0.015 & 0.78 & 0.26 & $>200$ & 1.8 & $>200$ & $>200$ & $>200$ & 200 & 0.26 & NT & NT \\
\hline
\end{tabular}

For compounds with a MIC within the detectable range, data are an average of three independent experiments. $\mathrm{CBN}=\mathrm{Carbenicillin}$; $\mathrm{CPR}=$ Ciprofloxacin; NVB $=$ Novobiocin; SMX $=$ Sulfamethoxazole; TMP $=$ Trimethoprim; NT $=$ Not tested.

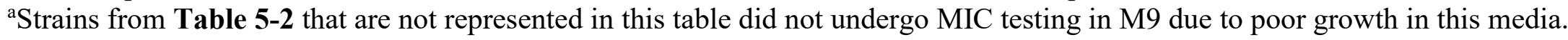

Table 5-4. MICsos of $E$. coli CETSA panel in LB media $(\mu M)$.

\begin{tabular}{lcccccccc}
\hline & \multicolumn{3}{c}{ Controls } & & \multicolumn{3}{c}{ DHFR targeting } \\
\cline { 2 - 4 } \cline { 6 - 8 } E. coli Strain & CBN & CPR & NVB & & TMP & UCP1195 & UCP1218 \\
\hline BW25113 & 9.4 & 0.081 & 83.3 & & 5.2 & 6.3 & $>50$ \\
tolC Kan-S & 1.6 & 0.0053 & 0.34 & & 0.39 & 0.0015 & 6.3 \\
$\Delta$ tolC Kan-S pPJ131:folP & $>200$ & 0.0076 & 0.39 & & 0.39 & 0.0015 & 6.3 \\
tolC Kan-S pPJ131:folA & $>200$ & 0.0061 & 0.39 & & 6.3 & 0.20 & $>6.3$ \\
\hline
\end{tabular}

$\mathrm{CBN}=$ Carbenicillin; $\mathrm{CPR}=$ Ciprofloxacin NVB $=$ Novobiocin $; \mathrm{TMP}=$ Trimethoprim. 
strain backgrounds.

When lysates of the complete CETSA panel were run side by side, a single band around $20 \mathrm{kDa}$ was detected for both FLAG-DHFR expressing strains, as well as a band just above $30 \mathrm{kDa}$ for the two FLAG-DHPS expressing strains (Figure 5-3). Neither of these bands was present either in lysate from the parent strains or in the strains harboring the empty plasmid. Some background bands were apparent in all strains but were for the most part consistent among all samples tested.

\section{Establishment of CETSA melting curves for DHFR and DHPS}

To obtain the optimal temperature gradient to achieve a CETSA melt curve for $E$. coli expressing each protein of interest, CETSA was first tested on a wide heat gradient ranging from $37^{\circ} \mathrm{C}$ to $70^{\circ}$ Celsius (Figure 5-4). TMP and SMX were included as controls for DHFR and DHPS, respectively. After drugging, the cells were washed and subjected to a CETSA heat gradient. The cells were then lysed and aggregated proteins removed by centrifugation. The resulting lysates were analyzed via $\alpha$ FLAG western blotting. Relative band intensities were calculated from the resulting bands. Using the Boltzmann sigmoidal nonlinear regression analysis to fit curves onto the data, aggregation temperatures $\left(\mathrm{T}_{\mathrm{AGG}}\right)$ could be determined at which $50 \%$ stabilization of the target protein occurred. Using this analysis, TMP stabilized DHFR by 4.5 degrees

(Figure 5-4A). SMX, however, showed negligible thermostabilization of DHPS in this assay (Figure 5-4B). Similar to the results seen with SPR, this could be due to the nature of the interaction of sulfonamides with DHPS (Table 5-1 and Figure 5-2). The ability to generate aggregation curves for both strains as well as the noticeable TMP-induced thermostabilization of DHFR yielded the first indication that this model can successfully detect target engagement in the intact GN cell system.

\section{DHPS lysate CETSA reveals antifolate affinity profile}

In vitro inhibitor binding assays are optimized for accuracy, reproducibility, and adequate signal. However, the optimal conditions necessary for quality in vitro data are often very different from the physiological environment of the target cell milieu, which is rich in other proteins that can modify both the inhibitor and the target of interest. Therefore, the observed fate and binding activity of inhibitors can vary vastly between these two environments.

To explore potential differences in antifolate binding between these two unique conditions, the lysate CETSA was employed to compare with the in vitro SPR results. This method involves drugging prepared lysates instead of intact cells, then subjecting the lysate samples to similar gradient heating, sample preparation, and immunodection similar to standard CETSA techniques. This allows for ligand-induced thermostabilization to be studied in the native environment in the absence of cell membranes, transport machinery, and efflux systems, which can all interfere with the 


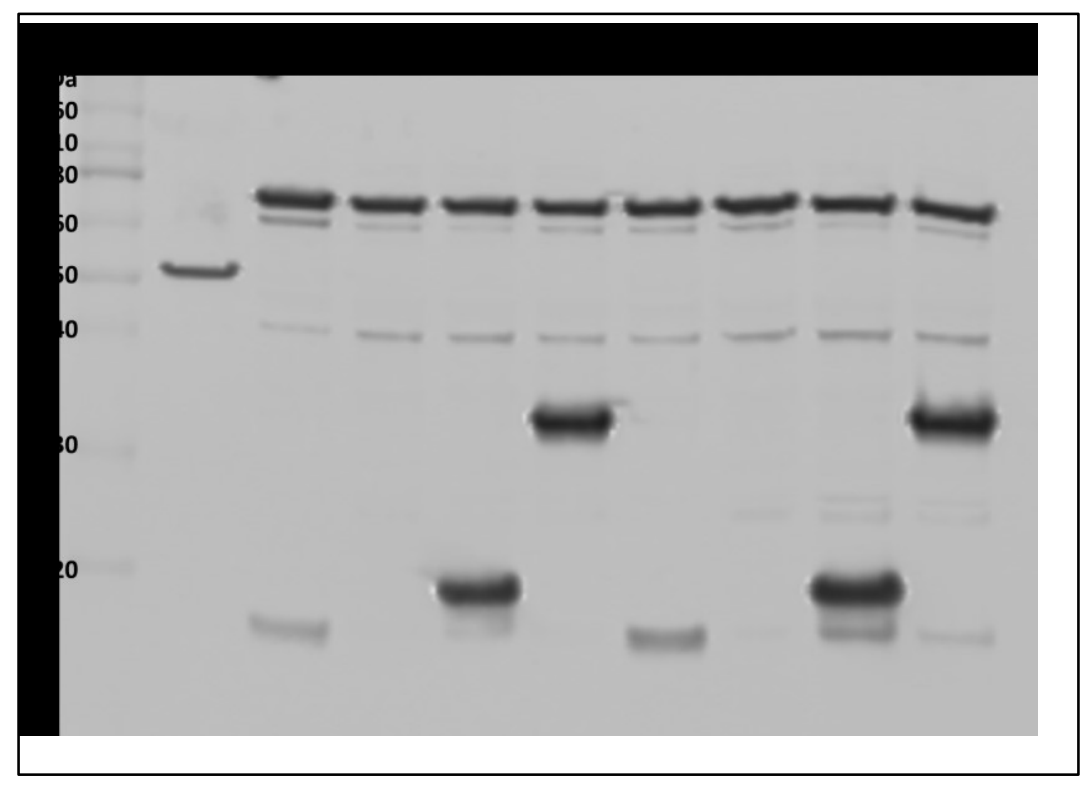

Figure 5-3. Western blot of lysates prepared from full CETSA panel and background strains.

For every lysate sample (lanes 2-10), approximately $50 \mu \mathrm{g}$ lysate was loaded for all lysate samples with the exception of the folP samples, which were approximately $100 \mu \mathrm{g}$. 1.5 $\mu$ L Novex Sharp Pre-stained Protein Ladder 2. 20 ng FLAG-BAP fusion protein control. 3. BW25113 parent strain 4. BW25113 pPJ131 empty plasmid 5. BW25113 pPJ131:folA 6. BW25113 pPJ131:folP 7. $\Delta$ tolC Kan-S parent strain 8. $\Delta$ tolC KanS pPJ131 empty plasmid 9. $\Delta$ tolC Kan-S pPJ131:folA 10. $\Delta$ tolC Kan-S pPJ131:folP. 


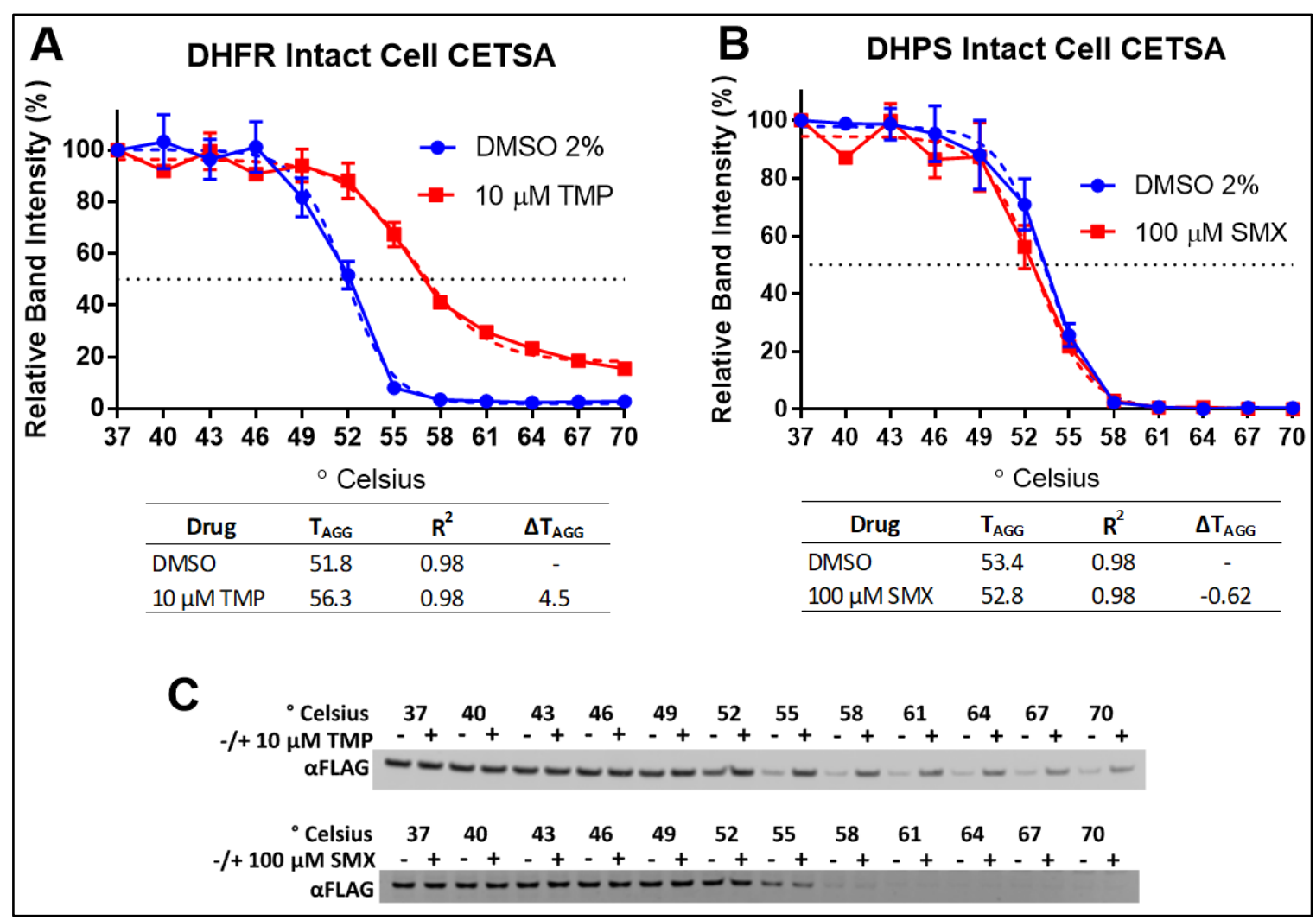

Figure 5-4. Preliminary whole cell CETSA covering a broad temperature range. For panels $\mathrm{A}$ and $\mathrm{B}$, aggregation curves are shown along with $\mathrm{T}_{\mathrm{AGG}}$ values calculated using the Boltzmann sigmoidal nonlinear regression fit. The fitted curves are represented by dashed colored lines and a dashed black line represents $50 \%$ relative band intensity on each graph. The data is representative of two technical replicates, and error bars represent the SEM. A. CETSA curve for E. coli expressing DHFR. B. CETSA curve for $E$. coli DHPS. C. Bands from $\alpha$ FLAG immunodetection. 
ability of the inhibitor to engage its target (Silver 2016).

Lysates from $E$. coli BW25113 and $\Delta$ tolC Kan-S strains expressing the folP gene were prepared and drugged at $100 \mu \mathrm{M}$, after which they were subject to a six-point heat gradient encompassing the aggregation curve of DHPS. A similar pattern of binding affinity to the in vitro SPR experiments was seen when comparing the resulting lysate CETSA curves with each DHPS inhibitor (Table 5-1 and Figure 5-5). The CETSA profiles were very similar between BW25113 and $\Delta$ tolC Kan-S. SMX again showed no thermostabilization of DHPS relative to the DMSO control. 1532 continued to show the strongest interaction, stabilizing beyond the detectable range for each strain. 1462 also shifted $\mathrm{T}_{\mathrm{AGG}}$ values approximately ten degrees for each strain. In contrast to the SPR results, MANIC showed slightly stronger stabilization than 3893 (Table 5-1). 3894 had the lowest thermostabilization of all pyridazines tested. The pterin-sulfa 568 showed a weak yet detectable increase in the DHPS $\mathrm{T}_{\mathrm{AGG}}$ (Figure 5-5).

The similar affinity profiles between lysates prepared in the BW25113 and the $\Delta$ tolC Kan-S background ensured a homogenous testing environment between the two genetically distinct strains in the absence of the cell membrane and associated efflux machinery. Furthermore, the similar affinity profiles observed during SPR testing ensured in vitro testing is predictive of DHPS binding interactions in a physiological environment.

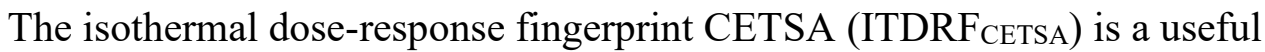
technique to study the effects of drug concentration on target stabilization (Martinez 2013). Lysate or cell preparations are drugged at varying concentrations, followed by heating at a constant temperature. To study the effect of concentration on antifolate

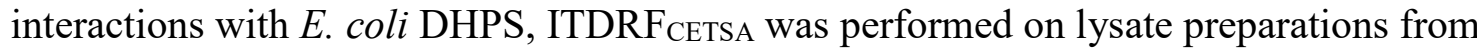
both strains expressing FLAG-DHPS at a fixed temperature of $58^{\circ}$ Celsius (Figure 5-6). Using the same curve-fit analysis as for the previous CETSA experiments, an ITDRF EC50 was determined for each inhibitor, which represents the concentration at which $50 \%$ stabilization of DHPS occurred relative to the highest concentration tested.

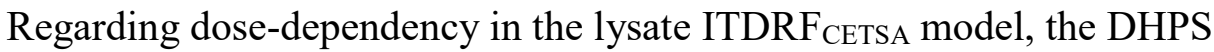
inhibitors had the exact same ranking as for SPR. 1532 again had the highest potency

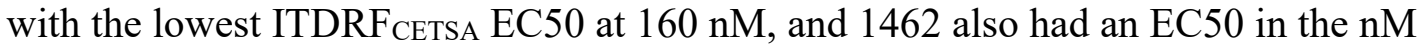
range. All other compounds had EC50s in the $\mu \mathrm{M}$ range, and SMX continued to not show binding effects in this model.

The similarities in interactions with the DHPS enzyme in the in vitro SPR assay,

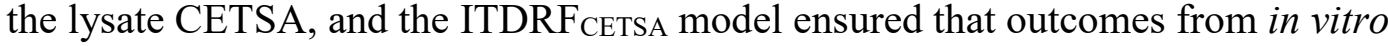
methods track expected outcomes from models of target engagement in the native

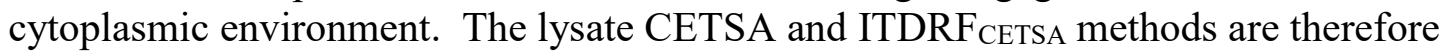
successful initial techniques to study target stabilization and dose-dependency of inhibitor binding without interference from issues with cell membrane permeability or efflux, which can be addressed in later experiments. 


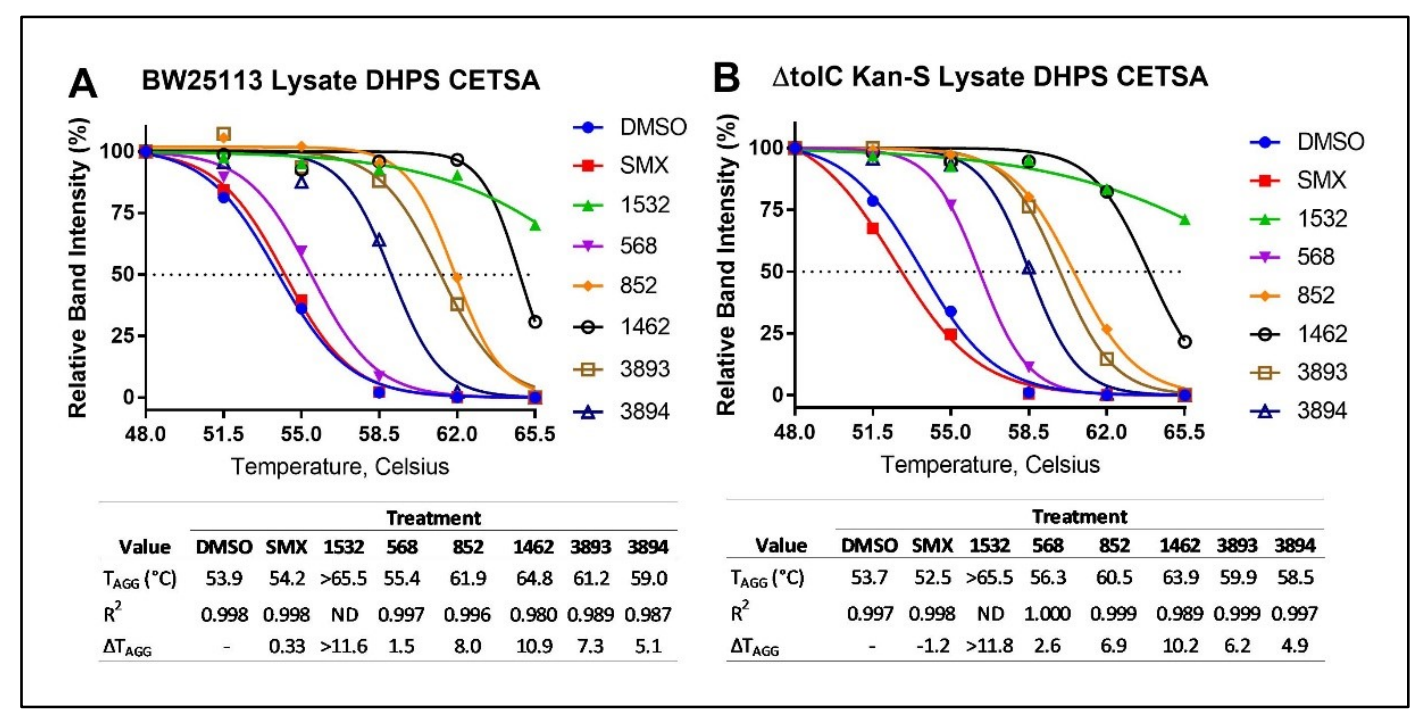

\section{Figure 5-5. Lysate DHPS CETSA.}

CETSA aggregation curves and calculated aggregation temperatures $\left(\mathrm{T}_{\mathrm{AGG}}\right)$ for prepared lysates for the BW25113 strain expressing FLAG-DHPS (A) and the $\Delta$ tolC Kan-S strain expressing FLAG-DHPS (B).

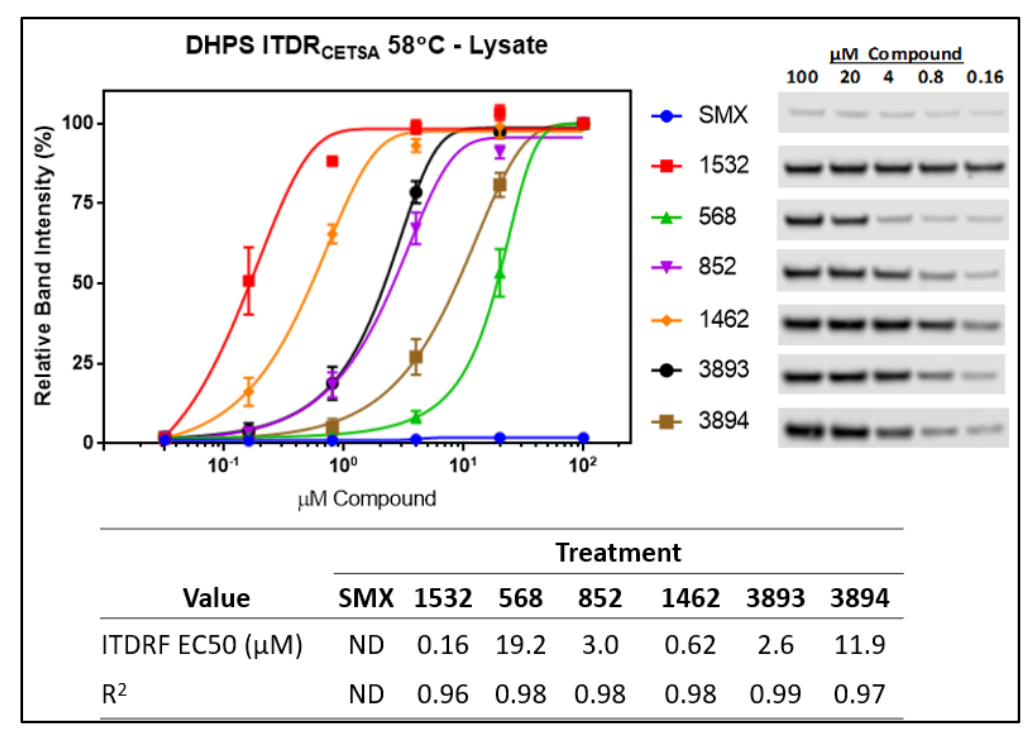

Figure 5-6. DHPS lysate ITDRFCETSA.

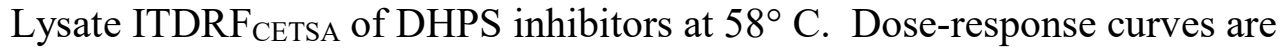
representative of three independent experiments. Error bars represent the SEM. The $\alpha$ FLAG western blot of the DHPS bands and the EC50s are also shown. 


\section{Addressing permeability issues with pyridazine inhibitors of DHPS}

Although deficiencies in cell entry and efflux susceptibility interfere with detection of target interactions, they ultimately affect in vivo activity and are therefore undoubtedly very important qualities to assess early-on. Therefore, the CETSA model was further employed to study the DHPS inhibitors in intact E. coli cells. To explore target engagement in the GN cell system, whole cell CETSA was performed on DHPS expressing strains treated with $100 \mu \mathrm{M}$ of each DHPS inhibitor (Figure 5-7). All DHPS inhibitors except 3893 only showed slight stabilization of DHPS within the intact cell of about $2^{\circ}$ Celsius. 3893, however, stood out as having superior binding affinity in the intact cell system showing a $\Delta \mathrm{T}_{\mathrm{AGG}}$ relative to the DMSO group of about $8^{\circ}$ Celsius for each strain. Although it was promising to find a pyridazine with improved cell permeability relative to previously established compounds, this effect was surprising considering 3893 is the precursor to 3894 , which was designed to have a primary amine moiety to accommodate GN entry (Richter 2017) (Figure 5-1). The effect of all other pyridazines showing very little stabilization compared to the lysate experiments suggests cytoplasmic cell permeability could be the major barrier to target engagement in the intact GN cell. RND pump-mediated efflux did not appear to affect the DHPS inhibitors, as the $\Delta \mathrm{T}_{\mathrm{AGG}}$ values were very similar between the BW25113 and $\Delta$ tolC Kan-S strains (Figure 5-7B).

\section{Global transcriptomic effects of antifolates}

Although 3893 was discovered as the first pyridazine showing signs of substantial DHPS stabilization in the intact cell CETSA, this compound still had no MIC in E. coli (Table 5-3). Although the pyridazines engage the same enzymatic target as sulfonamides, the MOA is likely distinct. Sulfonamides undergo a false product MOA while pyridazines inhibit DHPS directly and likely do not form a false product (Zhao 2012, Palmer 2014). The pterin-sulfa conjugates, however, engage both pterin and sulfonamide/ $p$ ABA sites simultaneously and have an MIC against $E$. coli, suggesting that the DHPS-mediated false product reaction is not essential for growth inhibition, but that either direct inhibition of DHPS or the false product itself can result in growth inhibition (Zhao 2016). DHPS is situated in the metabolically significant FBP and disruption of its normal function may have an associated transcriptomic signature, which may be dependent on whether native substrates are fluxed into the metabolically useless false product reaction. Transcriptional changes in several genes responsible for distinct steps of bacterial metabolism have been characterized for other antifolates (Nixon 2014). Comparison of the transcriptomic effect between sulfonamides, pterin-sulfas, and pyridazines may indicate different impacts on bacterial metabolism as well as the MOA of the inhibitors.

Treatment with 568 and 3893, however, showed interrogation of metabolically important pathways (Figure 5-8). There was an interesting overlap with the transcriptomic effect of the CysK inhibitor 3866 with both of these DHPS inhibitors, as genes involved in cysteine biosynthesis and sulfur metabolism were jointly altered. 


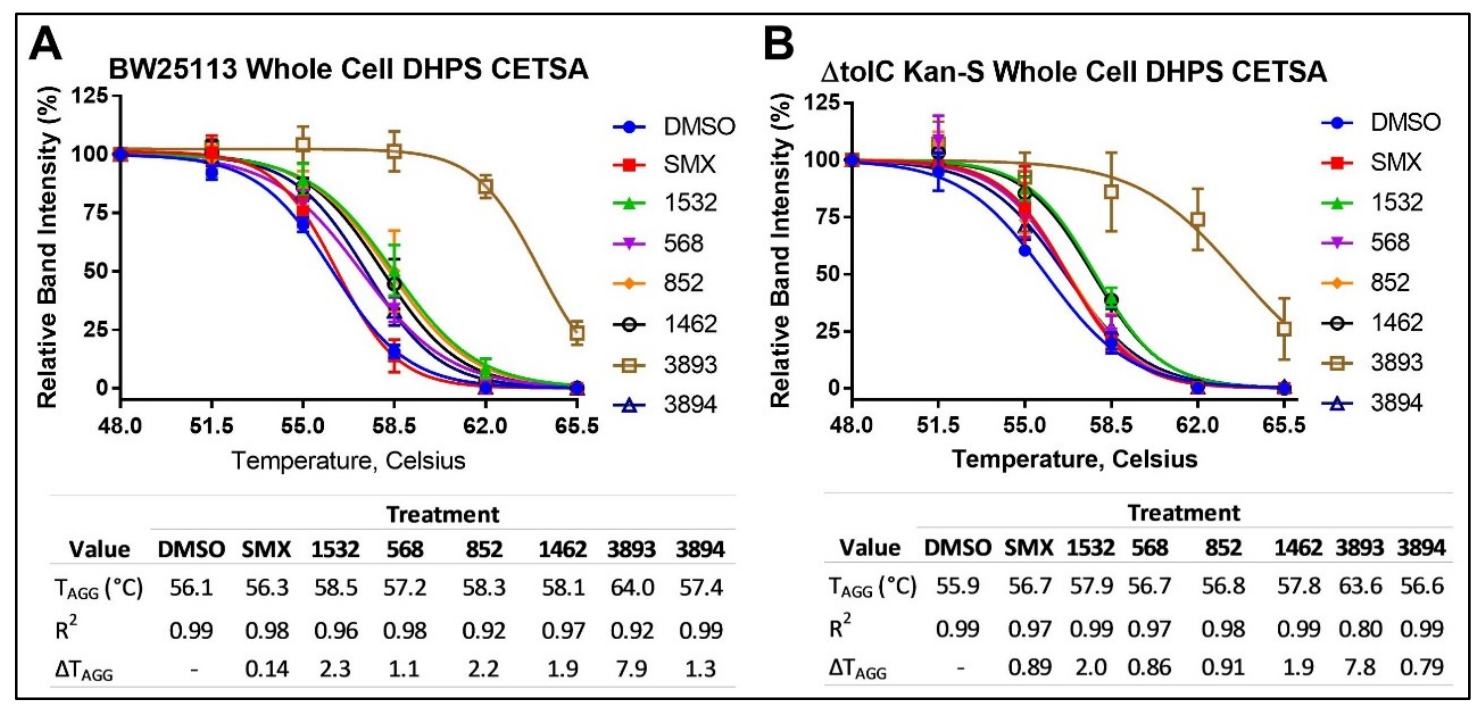

\section{Figure 5-7. Whole cell DHPS CETSA.}

CETSA curves are representative of three independent experiments for BW25113 and two independent experiments for $\triangle$ tolC Kan-S. Error bars represent the SEM. A. Intact cell CETSA curve for BW25113 expressing DHPS. B. Intact cell CETSA curve for $\Delta$ tolC Kan-S expressing DHPS. 


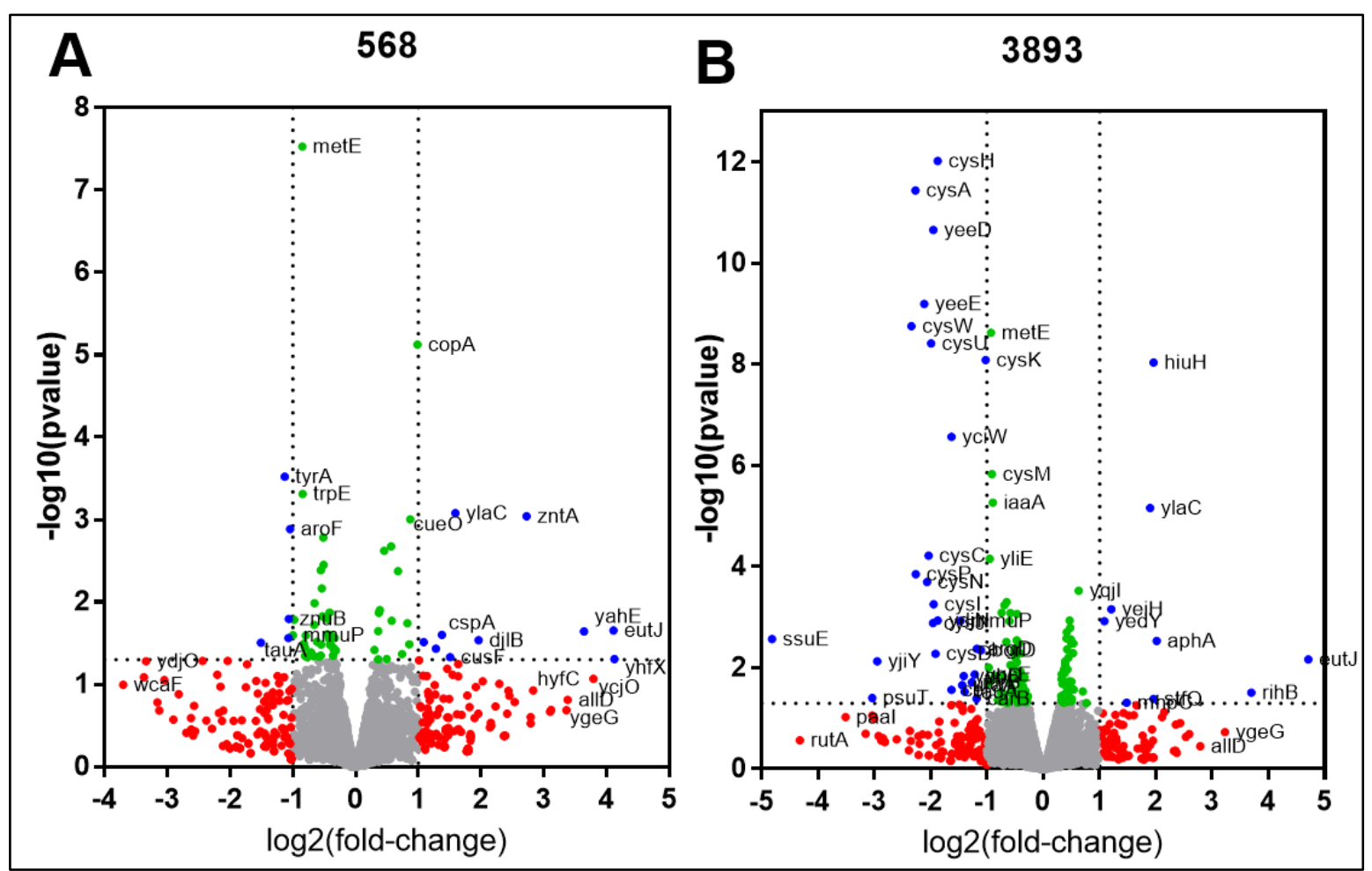

Figure 5-8. Volcano plots of RNA-seq study of $E$. coli treated with 568 and 3893. Dashed lines represent the cutoff for a significant change in gene transcription of twofold as well as a P-value of 0.05 . Genes with a significant transcriptional change and Pvalue are in blue, genes with a significant change in transcription but a P-value of $>0.01$ are in red. Green dots represent genes with insignificant changes in terms of both transcriptional fold-change and P-value. Grey dots represent genes with a P-value $>0.01$ and no significant transcription change. A. Transcriptomic effect of treatment with 146 $\mu \mathrm{M} 568$, and B. $100 \mu \mathrm{M} 3893$. 
Furthermore, 568 treatment resulted in the dysregulation of the $\operatorname{aroF}$, tyrA, and tyr $P$ genes involved in aromatic amino acid metabolism, which were also affected in response to 3866 treatment (Figure 4-11B). The downregulation of cbl, $s s u E$, and several genes within the cys operon in 3893 was an interesting effect. In fact, 3866 shared more genes with 3893 than the two DHPS inhibitors, 568 and 3893, share with each other (Figure 59). Half of these genes were involved in sulfur metabolic processes, including the $c y_{s} K$ gene itself. Although further experiments are required to understand this shared transcriptomic effect, this finding further indicates a shared metabolic effect between inhibition of both folate and cysteine biosynthesis initially evidenced in Chapter 4 .

\section{Assessing susceptibility to efflux with novel DHFR inhibitors}

Both PLAs showed sensitivity to efflux, with much lower MICs in the tolC knockout strains (Table 5-4). TMP also seemed to be sensitive to efflux to a more modest extent. The whole cell CETSA was performed with these compounds on both the parent and the $\Delta$ tolC Kan-S strains constitutively expressing FLAG-DHFR to see if susceptibility to efflux could also be observed using the CETSA assay (Figure 5-10). The $\Delta \mathrm{T}_{\mathrm{AGG}}$ values were compared to see if the presence of the TolC efflux pump subunit influenced the extent of thermostabilization of each compound in the intact cell. The $\Delta \mathrm{T}_{\mathrm{AGG}}$ for the $\Delta$ tolC Kan-S CETSA was consistently greater in comparison to the parent strain for all three DHFR-targeted compounds, which agreed with the increased susceptibility seen in the MIC assays (Table 5-4 and Figure 5-10).

To ensure the increased stabilization in the $\Delta$ tolC Kan-S strain was associated with efflux, the lysate CETSA was performed on both strains. For TMP and UCP1195, the $\Delta \mathrm{T}_{\mathrm{AGG}}$ values were very similar between lysates prepared from both strains (Figure 5-11). For all compounds tested, the increase in $\Delta \mathrm{T}_{\mathrm{AGG}}$ in the $\Delta \mathrm{TolC}$ Kan-S strains relative to the BW25113 strain was larger in the whole cell CETSA than in the lysate CETSA, indicating the intact efflux machinery in the whole cell preparation has a detectable effect that is less pronounced in the lysate preparation. In this CETSA model, UCP1195 seems to be more sensitive to efflux, which is reflective of the dramatic differences in susceptibility between the parent and TolC knockout strain during MIC testing (Table 5-4).

\section{Conclusions and Future Perspectives}

New inhibitors of GN bacteria are needed now more than ever. Early-stage inhibitors must be evaluated for qualities that predict in vivo performance as GN antimicrobials. CETSA shows utility as one of these early-stage tools to readily determine target engagement in intact GN cells using standard molecular biology methods. Although CETSA allows the study of target engagement within the intact cell, it does not provide quantitative information on the concentration of inhibitor accumulating inside the cell. Following previously established methods (Richter 2017), 


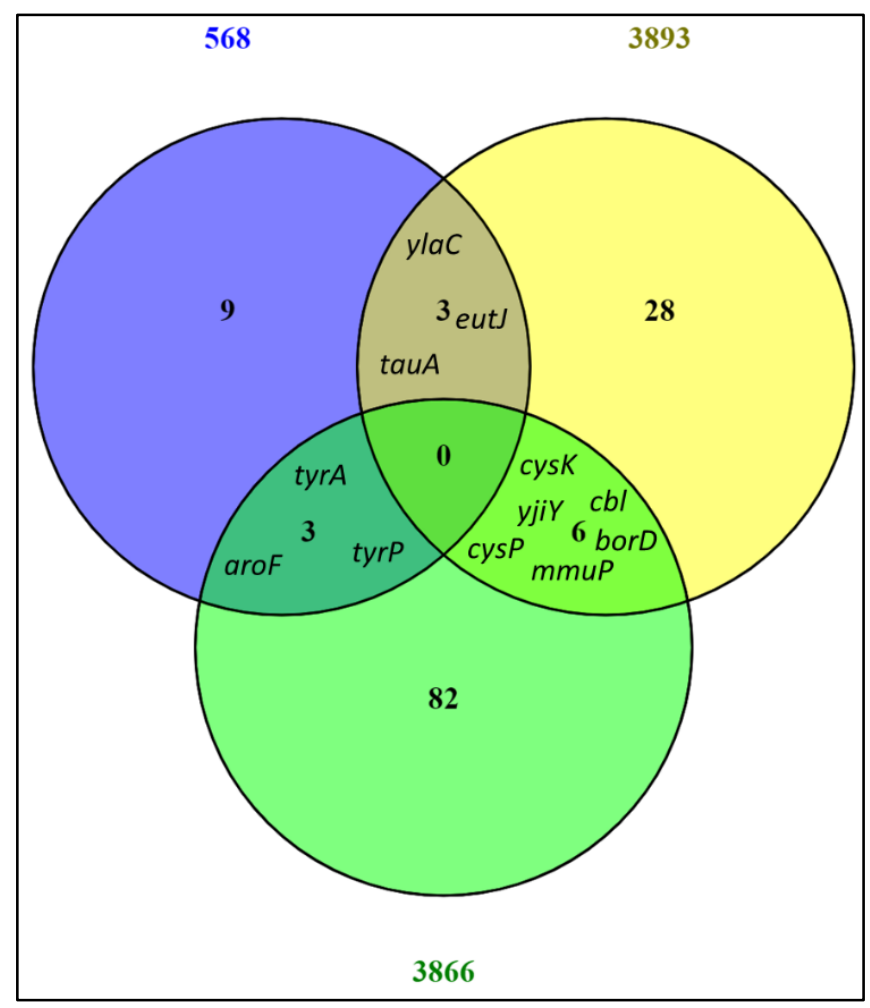

Figure 5-9. Venn diagrams of shared genes transcriptionally altered between DHPS inhibitors and the CysK inhibitor 3866.

Genes with at least a two-fold change in expression and a P-value of $\leq 0.05$ were compared between the two groups treated with DHPS inhibitors 568 and 3893 with the group treated with the CysK inhibitor 3866 (Chapter 4). The Venny tool was used to generate the Venn diagram (Oliveros 2015). 


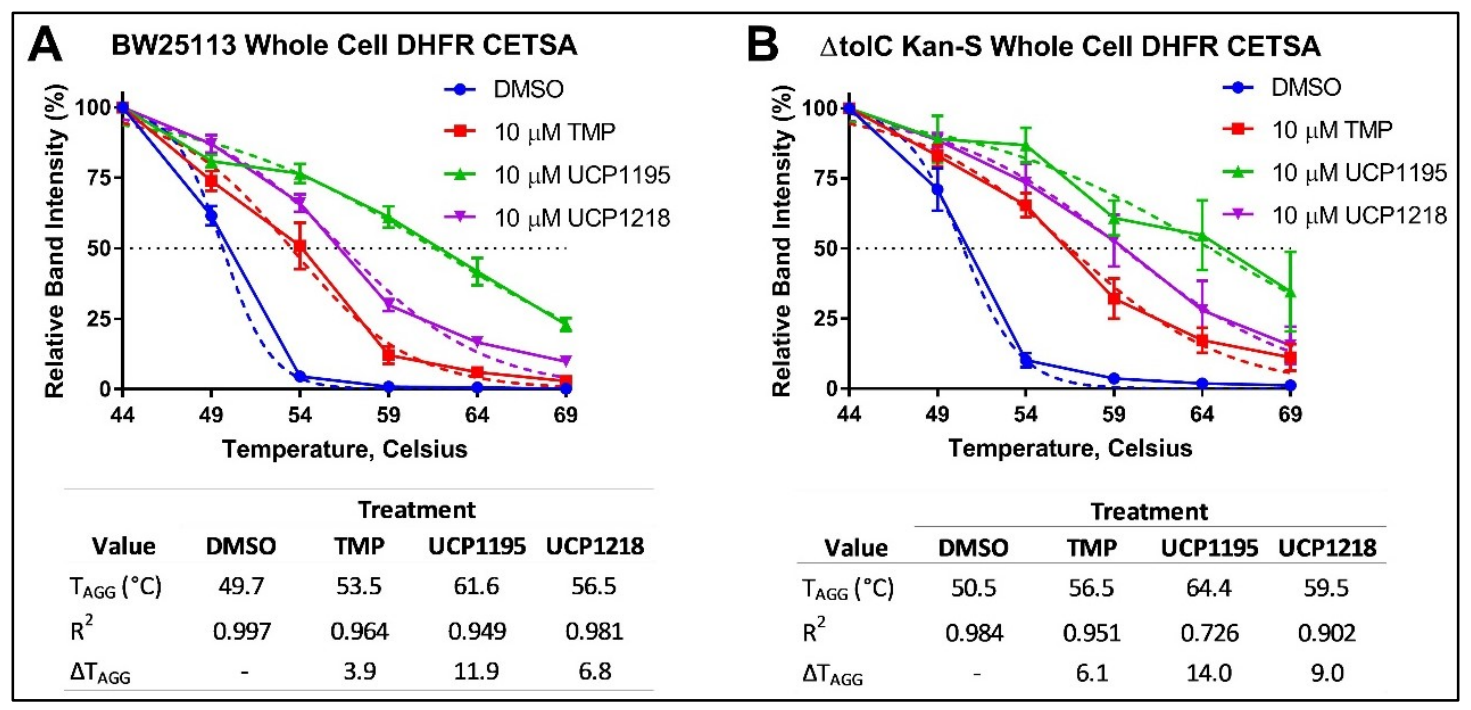

Figure 5-10. Whole cell DHFR CETSA.

Data are representative of three independent experiments. Error bars represent SEM. A. Whole cell CETSA profile for BW25113 expressing DHFR. B. Whole cell CETSA profile for $\Delta$ tolC Kan-S expressing DHFR.

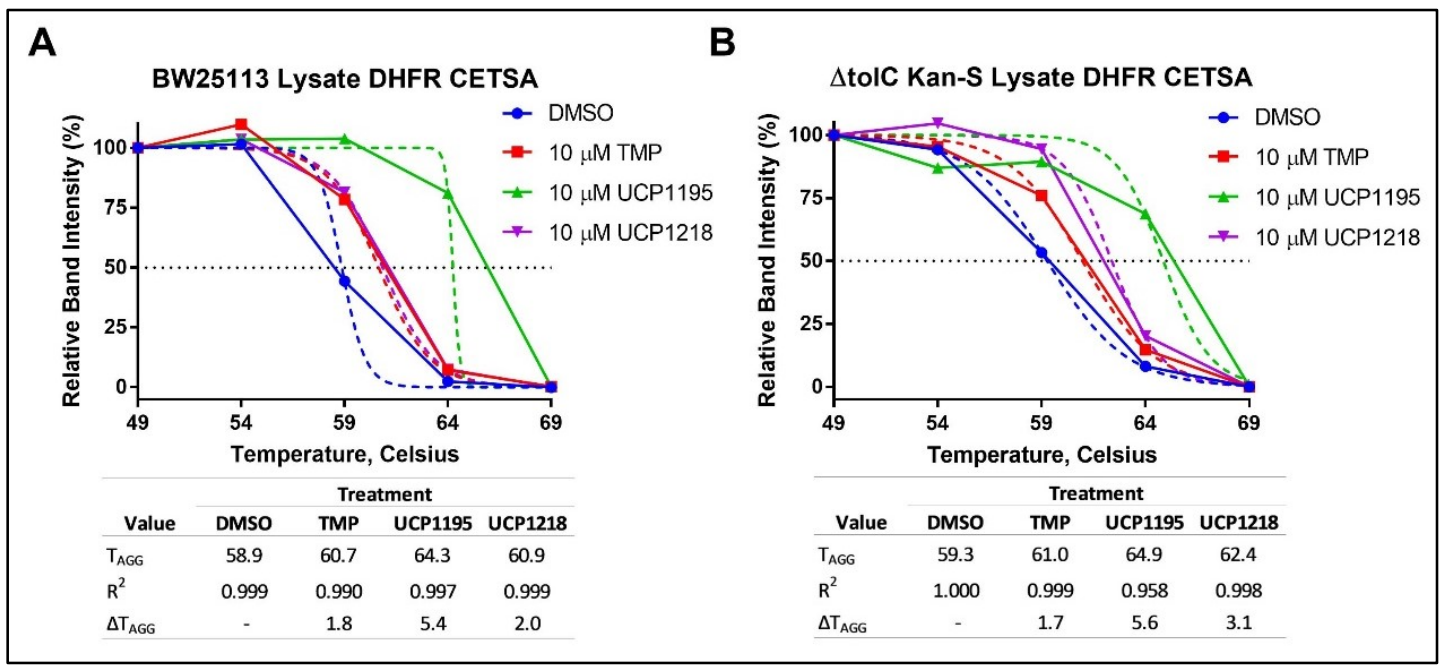

Figure 5-11. Lysate DHFR CETSA.

CETSA aggregation curves and calculated $\mathrm{T}_{\mathrm{AGG}}$ values are shown for lysates preparations from BW25113 (A) and $\Delta$ tolC Kan-S (B). 
orthologous investigations are currently ongoing using liquid chromatography tandem mass spectrometry to compare $E$. coli compound accumulation with the observed CETSA thermostabilization profiles for the compounds studied. This will provide another earlystage tool to understand how the SAR of pyridazines affects accumulation into the cell in addition to the cellular target engagement profiles obtained through the CETSA technique.

An important lesson from this study is that target engagement and affinity does not necessarily correlate with growth inhibition, even within whole cell studies. UCP1218 stabilized the $\mathrm{T}_{\mathrm{AGG}}$ of the BW25113 strain by nearly $7^{\circ}$ Celsius despite not having an MIC against this strain at the same concentration used for CETSA drugging (Table 5-4 and Figure 5-10). SMX did not show signs of biophysical interactions during both SPR and CETSA assays, yet it maintains a very low MIC. Furthermore, the pyridazines have $\mathrm{nM}$ affinity within the SPR assay, yet there is still no observable MIC. The DHPS stabilization observed with 3893 in the intact cell CETSA suggests intracellular target engagement can be improved, but 3893 has a KD approximately 45 times higher than then lead pyridazine 1532. Investigations are ongoing towards creating pyridazines that are both high potency and cell permeable.

Although pyridazine 3893 was discovered as showing the best intracellular target engagement of DHPS, further experimentation is required to understand the discrepancy between target engagement and biological activity. RNA-seq analysis revealed potential effects on sulfur metabolism that are shared with the CysK inhibitor 3866. Unfortunately, a major roadblock was the lack of transcriptomic effects seen with SMX treatment (data not shown). Whether this is due to the media, drug concentration, or if there simply is not a strong transcriptomic response associated with sulfonamide treatment is currently being investigated. However, an ongoing effort is to study the transcriptomic effects of DHPS inhibitors in combination with TMP. Sulfonamides are nearly always co-prescribed with TMP in the co-trimoxazole combination, and each drug is much more potent in combination (Bermingham 2002). Therefore, sulfonamides may have a more widespread and substantial impact on the $E$. coli transcriptome when an additional enzyme within the FBP is inhibited.

Within this study, CETSA showed promise as an early stage tool to track permeability, efflux, and intracellular target engagement in the GN cell. The standard methods required for CETSA with an immunodetection readout were amenable to the bacterial system. The use of a constitutive expression vector expressing FLAG-tagged target proteins accommodated immunodetection using commercially available antibodies and a single western blot protocol for both proteins. Stabilization by both established and experimental antifolates could be detected in both the standard CETSA and the

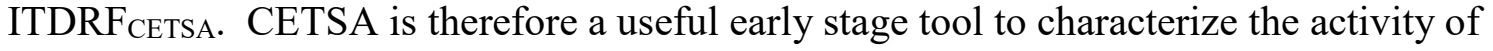
antimicrobial candidates in the GN intact cell system.

CETSA also holds much potential for medium- and high-throughput screening for antimicrobial compounds using alternative detection modalities to the traditional western blot. Time-resolved fluorescence resonance energy transfer (TR-FRET) and the 


\begin{abstract}
AlphaScreen rely on antibodies and/or affinity reagents that specifically recognize properly folded proteins, which eliminates the need for separating soluble from aggregated components of the lysates (Jafari 2014). Furthermore, various reporter-based readouts that do not rely on antibodies have been recently developed for CETSA to eliminate the intensive optimization required for immunodetection (McNulty 2018, Martinez 2018). These developments inspire much promise towards using CETSA to address crucial qualities of antibacterial agents during both screening and lead development stages.
\end{abstract}




\section{CHAPTER 6. CONTRIBUTIONS TO THE FIELD AND LOOKING FORWARD}

This dissertation aims to improve the field of antimicrobial discovery from three major angles, including improved understanding of resistance mechanisms to guide inhibitor development, the discovery of new antimicrobials disrupting bacterial metabolism, and improvement of methods to gauge the fate of inhibitors in the GN cell system. All three are studied in the context of bacterial metabolism.

Improvements to the understanding of sulfonamide resistance mechanisms were presented in Chapter 2, which guides the development of new DHPS inhibitors that are impervious to circulating resistance mechanisms. Reported variations in the amino acid sequence of DHPS among antifolate-resistant $S$. aureus strains were used to investigate the basis of sulfonamide resistance in this clinically important pathogen. Unexpected roles in resistance among secondary mutations were revealed, and nearly all of the mutations studied had an apparent effect on regions extending beyond the substrate envelope, which appears most vulnerable to mutation. This has guided the focus of DHPS inhibitor development to avoid engaging these areas of the enzyme. Most of these mutations are shared among other pathogenic species, so this information benefits inhibitor design towards a range of clinically significant pathogens.

Another aim of this dissertation is to improve methods used in early-stage antibiotic drug discovery, which are addressed in Chapters 3 and 5. During the drug discovery process, standard biological testing conditions select for a limited number of bacterial targets. Conventional testing media is often nutrient rich to ensure fast and optimal growth of the organisms studied, however nutrients within the testing media can mask the inhibitory effect of various antimetabolites. Therefore, many antimetabolite inhibitors and new metabolic targets have likely gone undetected in previous HTS campaigns. In Chapter 3, it was discovered that nutrient-restricted broth is required for measurement of the biological activity among next-generation antifolates, and supplementation with metabolites related to the target pathway increased the MIC. Testing in both nutrient-restricted and supplemented conditions was an effective strategy both for the detection of biological activity and to ensure an antimetabolite MOA. These methods were adapted to an $E$. coli whole cell screen for new antimetabolite compounds, leading to the discovery of several exciting candidate molecules for drug repurposing as well as small molecule inhibitors yet to be characterized as antimicrobials. Targeted whole cell screening in selective media followed by MOA determination is gaining stamina in recent years due to the wider range of targets discovered along with first-inclass inhibitors that are already whole-cell effective. Ultimately these approaches will hopefully yield first-in-class antimetabolite inhibitors that will bolster the current antibiotic armamentarium.

Improvement of antibiotic discovery methods is also addressed in Chapter 5 with a focus on developing strategies to track the fate of inhibitors in the GN cell system, which is notoriously difficult to drug. The CETSA technique was adapted to explore important antimicrobial qualities in this cell system including target engagement in the 
intact cell and efflux. The use of CETSA as an early stage tool for antibiotic discovery is not as developed as for targets in the eukaryotic cell system, but the methods are amenable to either cell type. The CETSA methods were adapted to the E. coli cell system and were used to characterize inhibitors of both DHPS and DHFR within the FBP. This analysis allowed for the discovery of 3893 as having the best engagement of DHPS in the intact GN cell system. Furthermore, DHFR inhibitors showed increased stabilization of the intracellular target in an E. coli $\Delta$ tolC strain, which confirmed their susceptibility to efflux. The CETSA method continues to serve as an early stage tool to quickly gauge performance in whole cells among new inhibitors. This method has the potential to greatly benefit other drug discovery campaigns as new classes of GNeffective antibiotics are desperately needed.

The final contribution of this dissertation to the field of AMR is the discovery of a new series of $E$. coli inhibitors targeting CysK. The current antibiotic arsenal generally targets a limited set of cell processes, and cysteine biosynthesis represents a new target that is absent in humans and positioned at the juncture of multiple metabolic pathways. The TAT series was discovered in the HTS from Chapter 3 and further characterized in Chapter 4. Microbial metabolism has garnered interest in recent years among the antibiotic drug discovery community, and the TAT target CysK has been evaluated for inhibitor development among several pathogenic species. Similar to the historically successful antifolate antibiotics, which take advantage of a metabolic pathway unique to prokaryotes and lower eukaryotes, targeting other metabolic branches such as cysteine biosynthesis is a promising strategy for acquiring new inhibitors. In contrast to current inhibitor campaigns towards CysK, the mechanism of the TATs could likely occur through formation of a false product, which is analogous to the MOA of sulfonamides. In addition to interfering with the native function of the protein target, false product mechanisms can alter the available pools of related metabolites and disrupt regulatory processes. The TATs were found to have a widespread impact on the transcription of genes in several metabolic pathways beyond cysteine biosynthesis. Altogether, the unique antimetabolite MOA of the TATs, as well as the widespread impact on E. coli metabolism, yields much promise towards using small molecules to inhibit GN pathogens.

The insights garnered from this study will aid ongoing antibiotic discovery campaigns and foster interest in pursuing microbial metabolism for new inhibitor development. This dissertation revealed the exciting potential of bacterial metabolism as a rich source of new and effective antibiotic targets. During the study of metabolic targets for the development of novel and next-generation inhibitor series, a significant focus within this dissertation is maintained on shifting conventional paradigms in the drug discovery field that limit discovery and neglect key properties of candidate inhibitors that are critical for in vivo efficacy. Although the current AMR crisis is foreboding, advances outlined in this dissertation towards antibacterial drug discovery within new metabolic pathways show much promise towards fighting drug-resistant infections. 


\section{LIST OF REFERENCES}

Abraham EP, Chain E, Fletcher CM, Gardner DM, Heatley NG, Jennings MA, et al. Further observations on penicillin. The Lancet. 1941 Aug;238(6155)177-189.

Afonine PV, Grosse-Kunstleve RW, Echols N, Headd JJ, Moriarty, NW, Mustyakimov $\mathrm{M}$, Terwilliger TC, et al. Towards automated crystallographic structure refinement with phenix.refine. Acta Crystallogr D Biol Crystallogr. 2012;68(Pt 4):352-367.

Alanis AJ. Resistance to antibiotics: are we in the post-antibiotic era? Arch Med Res. 2005 Nov-Dec;36(6):697-705.

Amori L, Katkevica S, Bruno A, Campanini B, Felici P., Mozzarelli A., et al. Design and synthesis of trans-2-substituted-cyclopropane-1-carboxylic acids as the first nonnatural small molecule inhibitors of O-acetylserine sulfhydrylase. MedChemCommun 2012;3:1111-16.

Baba T, Ara T, Hasegawa M, Takai, Y., Okumura Y., Baba M, et al. Construction of Escherichia coli K-12 in-frame, single-gene knockout mutants: the Keio collection. Mol Syst Biol. 2006;2:2006.0008.

Babaoglu K, Qi J, Lee RE, White SW. Crystal structure of 7,8-dihydropteroate synthase from Bacillus anthracis: mechanism and novel inhibitor design. Structure. 2004 Sep;12(9):1705-17.

Baca AM, Sirawaraporn R, Turley S, Sirawaraporn W, Hol WG. Crystal structure of Mycobacterium tuberculosis 7,8-dihydropteroate synthase in complex with pterin monophosphate: new insight into the enzymatic mechanism and sulfa-drug action. J Mol Biol. 2000 Oct 6;302(5):1193-212.

Beinert H. A tribute to sulfur. Eur J Biochem. 2000 Sep;267(18):5657-64.

Bermingham A, Derrick JP. The folic acid biosynthesis pathway in bacteria: evaluation of potential for antibacterial drug discovery. Bioessays. 2002 Jul;24(7):637-48.

Bornemann C, Jardine MA, Spies HS, Steenkamp DJ. Biosynthesis of mycothiol: elucidation of the sequence of steps in Mycobacterium smegmatis. Biochem J. 1997;325 (Pt 3)(Pt 3):623-629.

Bowen AC, Carapetis JR, Currie BJ, Fowler V Jr, Chambers HF, Tong SYC. Sulfamethoxazole-trimethoprim (Cotrimoxazole) for skin and soft tissue infections including impetigo, cellulitis, and abscess. Open Forum Infect Dis. 2017;4(4):ofx232. 
Brogden RN, Carmine AA, Heel RC, Speight TM, Avery GS. Trimethoprim: a review of its antibacterial activity, pharmacokinetics and therapeutic use in urinary tract infections. Drugs. 1982 Jun;23(6):405-30.

Brown DG, May-Dracka TL, Gagnon MM, Tommasi R. Trends and exceptions of physical properties on antibacterial activity for Gram-positive and Gram-negative pathogens. J Med Chem. 2014 Dec 11;57(23):10144-61.

Brown ED. Is the GAIN Act a turning point in new antibiotic discovery? Can J Microbiol. 2013 Mar;59(3):153-6.

Campanini B, Speroni F, Salsi E, Cook PF, Roderick SL, Huang B, et al. Interaction of serine acetyltransferase with O-acetylserine sulfhydrylase active site: evidence from fluorescence spectroscopy. Protein Sci. 2005;14(8):2115-2124.

Centers for Disease Control and Prevention. Antibiotic resistance threats in the United States, 2013. Atlanta (GA): Centers for Disease Control and Prevention, U.S. Department of Health and Human Services; 2013.

Chain E, Florey HW, Gardner AD, Heatley NG, Jennings MA, Orr-Ewing J, et al. Penicillin as a chemotherapeutic agent. The Lancet. 1940;236(6104):226-228.

Copeland RA. Evaluation of enzyme inhibitors in drug discovery. A guide for medicinal chemists and pharmacologists. Methods Biochem Anal, 2005;46, 1-265.

Dallas WS, Gowen JE, Ray PH, Cox MJ, Dev IK. Cloning, sequencing, and enhanced expression of the dihydropteroate synthase gene of Escherichia coli MC4100. J Bacteriol. 1992;174(18):5961-5970.

Dassault Systèmes BIOVIA, Pipeline Pilot, Version 17.2. San Diego (CA): Dassault Systèmes, 2018.

Davis TD, Gerry CJ, Tan DS. General platform for systematic quantitative evaluation of small-molecule permeability in bacteria. ACS Chem Biol. 2014;9(11):2535-2544.

Dennis ML, Pitcher NP, Lee MD, DeBono AJ, Wang ZC, Harjani JR, et al. Structural basis for the selective binding of inhibitors to 6-hydroxymethyl-7,8-dihydropterin pyrophosphokinase from Staphylococcus aureus and Escherichia coli. J Med Chem. 2016 Jun 9;59(11):5248-63.

Dharavath S, Raj I, Gourinath S. Structure-based mutational studies of O-acetylserine sulfhydrylase reveal the reason for the loss of cysteine synthase complex formation in Brucella abortus. Biochem J. 2017 Mar 23;474(7):1221-1239.

Domagk G. Chemotherapie der bakteriellen infektionen. Angewandte Chemie. 1935;48(42):657-667. 
Dosselaere F, Vanderleyden J. A metabolic node in action: Chorismate-utilizing enzymes in microorganisms. Crit Rev Microbiol. 2001;27(2):75-131.

Durant JL, Leland B, Henry DR, Nourse JG. Reoptimization of MDL keys for use in drug discovery. J Chem Inf Comput Sci. 2002;42:1273-80.

Emsley P, Cowtan K. Coot: model-building tools for molecular graphics. Acta Crystallogr. D Biol. Crystallogr. 2004;60(Pt 12 Pt 1), 2126-2132

Farha MA, Brown ED. Drug repurposing for antimicrobial discovery. Nat Microbiol. 2019 Apr;4(4):565-577.

Ferla MP, Patrick WM. Bacterial methionine biosynthesis. Microbiology. 2014 Aug;160(Pt 8):1571-84.

Fermer C, Kristiansen BE, Sköld O, Swedberg G. Sulfonamide resistance in Neisseria meningitidis as defined by site-directed mutagenesis could have its origin in other species. J Bacteriol. 1995;177(16):4669-4675.

Fleming A. On the antibacterial action of cultures of a Penicillium, with special reference to their use in the isolation of B. influenzæ. Br J Exp Pathol. 1929;10(3):226-236.

Goldberg E, Paul M, Talker O, Samra Z, Raskin M, Hazzan R, et al. Co-trimoxazole versus vancomycin for the treatment of methicillin-resistant Staphylococcus aureus bacteraemia: A retrospective cohort study. J Antimicrob Chemother. 2010 Aug;65(8):1779-83.

Griffith EC. Structural considerations in antimicrobial drug design [dissertation]. Memphis (TN). The University of Tennessee Health Science Center; 2015.

Griffith OW. Mammalian sulfur amino acid metabolism: An overview. Methods Enzymol. 1987;143:366-76.

Griffith EC, Wallace MJ, Wu Y, Kumar G, Gajewski S, Jackson P, et al. The structural and functional basis for recurring sulfa drug resistance mutations in Staphylococcus aureus dihydropteroate synthase. Front Microbiol. 2018 Jul 17;9:1369.

Gyaneshwar P, Paliy O, McAuliffe J, Popham DL, Jordan MI, Kustu S. Sulfur and nitrogen limitation in Escherichia coli K-12: Specific homeostatic responses. J Bacteriol. 2005;187(3):1074-1090.

Hammoudeh DI, Daté M, Yun MK, Zhang W, Boyd VA, Follis AV, et al. Identification and characterization of an allosteric inhibitory site on dihydropteroate synthase. ACS Chem Biol. 2014;9(6):1294-1302. 
Hampele IC, D'Arcy A, Dale GE, Kostrewa D, Nielsen J, Oefner C, et al. Structure and function of the dihydropteroate synthase from Staphylococcus aureus. J Mol Biol. 1997 Apr 25;268(1):21-30.

Haselbeck R, Wall D, Jiang B, Ketela T, Zyskind J, Bussey H, et al. Comprehensive essential gene identification as a platform for novel anti-infective drug discovery. Curr Pharm Des. 2002;8(13):1155-72.

Hatzios SK, Bertozzi CR. The regulation of sulfur metabolism in Mycobacterium tuberculosis. PLoS Pathog. ;7(7):e1002036.

Heidari-Torkabadi H, Che T, Lombardo MN, Wright DL, Anderson AC, Carey PR. Measuring propargyl-linked drug populations inside bacterial cells, and their interaction with a dihydrofolate reductase target, by Raman microscopy. Biochemistry. 2015 May 5;54(17):2719-26.

Huang B, Vetting MW, Roderick SL. The active site of O-acetylserine sulfhydrylase is the anchor point for bienzyme complex formation with serine acetyltransferase. J Bacteriol. 2005 May; 187(9):3201-5.

Hughes WT. Use of dapsone in the prevention and treatment of Pneumocystis carinii pneumonia: a review. Clin Infect Dis. 1998 Jul;27(1):191-204.

Ikegami F, Kaneko M, Kaneko H, Murakoshi I. Purification and characterization of cysteine synthase from Citrullus vulgaris. Phytochemistry. 1988; 697-701.

Jafari R, Almqvist H, Axelsson H, Ignatushchenko M, Lundbäck T, Nordlund P, et al. The cellular thermal shift assay for evaluating drug target interactions in cells. Nat Protoc. 2014 Sep;9(9):2100-22.

Jean Kumar VU, Poyraz Ö, Saxena S, Schnell R, Yogeeswari P, Schneider G, et al. Discovery of novel inhibitors targeting the Mycobacterium tuberculosis O-acetylserine sulfhydrylase (CysK1) using virtual high-throughput screening. Bioorg Med Chem Lett. 2013 Mar 1;23(5):1182-6.

Kabsch W. XDS. Acta Crystallogr D Biol Crystallogr. 2010 Feb;66(Pt 2):125-32.

Kai M, Matsuoka M, Nakata N, Maeda S, Gidoh M, Maeda Y, et al. Diaminodiphenylsulfone resistance of Mycobacterium leprae due to mutations in the dihydropteroate synthase gene. FEMS Microbiol Lett. 1999 Aug 15;177(2):231-5.

Kessler D. Enzymatic activation of sulfur for incorporation into biomolecules in prokaryotes. FEMS Microbiol Rev. 2006 Nov;30(6):825-40.

Kredich NM. Biosynthesis of cysteine. In Escherichia coli and Salmonella (ed. F.C. Neidhardt). ASM Press. 1996;pp. 514-527. 
Kredich NM, Foote LJ, Hulanicka MD. Studies on the mechanism of inhibition of Salmonella typhimurium by 1,2,4-triazole. J Biol Chem. 1975 Sep 25;250(18):7324-31.

Kredich NM. The molecular basis for positive regulation of cys promoters in Salmonella typhimurium and Escherichia coli. Mol Microbiol. 1992 Oct;6(19):2747-53.

Kredich NM, Tomkins GM. The enzymic synthesis of L-cysteine in Escherichia coli and Salmonella typhimurium. J Biol Chem. 1966 Nov 10;241(21):4955-65.

Landy M, Larkum NW, Oswald EJ, Streightoff F. Increased synthesis of p-aminobenzoic acid associated with the development of sulfonamide resistance in Staphylococcus aureus. Science. 1943 Mar 19;97(2516):265-7.

Lever OW Jr, Bell LN, McGuire HM, Ferone R. Monocyclic pteridine analogues. Inhibition of Escherichia coli dihydropteroate synthase by 6-amino-5-nitrosoisocytosines. J Med Chem. 1985 Dec;28(12):1870-4.

Levy C, Minnis D, Derrick JP. Dihydropteroate synthase from Streptococcus pneumoniae: structure, ligand recognition and mechanism of sulfonamide resistance. Biochem J. 2008 Jun 1;412(2):379-88.

Lewis K. New approaches to antimicrobial discovery. Biochem Pharmacol. 2017 Jun 15;134:87-98.

Lewis K. Persister cells. Annu Rev Microbiol. 2010;64:357-72.

Lewis K. Platforms for antibiotic discovery. Nat Rev Drug Discov. 2013 May;12(5):37187.

Lombardo MN, G-Dayanandan N, Wright DL, Anderson AC. Crystal structures of trimethoprim-resistant dfra1 rationalize potent inhibition by propargyl-linked antifolates. ACS Infect Dis. 2016 Feb 12;2(2):149-56.

Magalhães J, Franko N, Annunziato G, Pieroni M, Benoni R, Nikitjuka A, et al. Refining the structure-activity relationships of 2-phenylcyclopropane carboxylic acids as inhibitors of O-acetylserine sulfhydrylase isoforms. J Enzyme Inhib Med Chem. 2018;34(1):31-43.

Martinez Molina D, Jafari R, Ignatushchenko M, Seki T, Larsson EA, Dan C, et al. Monitoring drug target engagement in cells and tissues using the cellular thermal shift assay. Science. 2013 Jul 5;341(6141):84-7.

Martinez NJ, Asawa RR, Cyr MG, Zakharov A, Urban DJ, Roth JS, et al. A widelyapplicable high-throughput cellular thermal shift assay (CETSA) using split Nano Luciferase. Sci Rep. 2018;8(1):9472. Published 2018 Jun 21. 
Maskell JP, Sefton AM, Hall LM. Mechanism of sulfonamide resistance in clinical isolates of Streptococcus pneumoniae. Antimicrob Agents Chemother.

1997;41(10):2121-2126.

Mazumder M, Gourinath S. Structure-based design of inhibitors of the crucial cysteine biosynthetic pathway enzyme o-acetyl serine sulfhydrylase. Curr Top Med Chem. 2016;16(9):948-59.

McCoy AJ, Grosse-Kunstleve RW, Adams PD, Winn MD, Storoni LC, Read RJ. Phaser crystallographic software. J. Appl. Cryst. 2007;40, 658-674.

McNulty DE, Bonnette WG, Qi H, Wang L, Ho TF, Waszkiewicz A, et al. A highthroughput dose-response cellular thermal shift assay for rapid screening of drug target engagement in living cells, exemplified using SMYD3 and IDO1. SLAS Discov. 2018 Jan;23(1):34-46.

Mei Q, Gurunathan S, Masur H, Kovacs JA. Failure of co-trimoxazole in Pneumocystis carinii infection and mutations in dihydropteroate synthase gene. Lancet. 1998 May 30;351(9116):1631-2.

Minato Y, Dawadi S, Kordus SL, Sivanandam A, Aldrich CC, Baughn AD. Mutual potentiation drives synergy between trimethoprim and sulfamethoxazole. Nat Commun. 2018 Mar 8;9(1):1003.

Mitscher LA. Antibiotics and antimicrobial agents. In: Foye WO, Lemke TL, Williams DA, editors. Principles of medicinal chemistry. Pennsylvania: Lippincott Williams \& Wilkins; 1995. p. 764-766.

Mori M, Jeelani G, Masuda Y, Sakai K, Tsukui K, Waluyo D, et al. Identification of natural inhibitors of Entamoeba histolytica cysteine synthase from microbial secondary metabolites. Front Microbiol. 2015 Sep 14;6:962.

Moriarty NW, Grosse-Kunstleve RW, Adams PD. Electronic Ligand Builder and Optimization Workbench (eLBOW): a tool for ligand coordinate and restraint generation. Acta Crystallogr. D Biol. Crystallogr. 2009;65, 1074-1080.

Mozzarelli A, Bettati S, Campanini B, Salsi E, Raboni S, Singh R, et al. The multifaceted pyridoxal 5'-phosphate-dependent O-acetylserine sulfhydrylase. Biochim Biophys Acta. 2011 Nov;1814(11):1497-510.

Nagpal I, Raj I, Subbarao N, Gourinath S. Virtual screening, identification and in vitro testing of novel inhibitors of O-acetyl-L-serine sulfhydrylase of Entamoeba histolytica. PLoS One. 2012;7(2):e30305.

Nikaido H, Takatsuka Y. Mechanisms of RND multidrug efflux pumps. Biochim Biophys Acta. 2009 May;1794(5):769-81. 
Nikiforova VJ, Bielecka M, Gakière B, Krueger S, Rinder J, Kempa S, et al. Effect of sulfur availability on the integrity of amino acid biosynthesis in plants. Amino Acids. 2006 Mar;30(2):173-83.

Nixon MR, Saionz KW, Koo MS, Szymonifka MJ, Jung H, Roberts JP, Nandakumar M, et al. Folate pathway disruption leads to critical disruption of methionine derivatives in Mycobacterium tuberculosis. Chem Biol. 2014 Jul 17;21(7):819-30.

Nozaki T, Ali V, Tokoro M. Sulfur-containing amino acid metabolism in parasitic protozoa. Adv Parasitol. 2005;60:1-99.

O’Neill J. Tackling drug-resistant infectious globally: Final report and recommendations (The Review on Microbial Resistance, 2016). 2016 May.

Oliveros JC. Venny. An interactive tool for comparing lists with Venn's diagrams. 2015 [accessed 22 ${ }^{\text {nd }}$ April 2019] Available from http://bioinfogp.cnb.csic.es/tools/venny/index.html (Accessed on 22 ${ }^{\text {nd }}$ April 2019)

Palmer AC, Kishony R. Opposing effects of target overexpression reveal drug mechanisms. Nat Commun. 2014 Jul 1;5:4296.

Payne DJ, Gwynn MN, Holmes DJ, Pompliano DL. Drugs for bad bugs: confronting the challenges of antibacterial discovery. Nat Rev Drug Discov. 2007 Jan;6(1):29-40.

Pornthanakasem W, Riangrungroj P, Chitnumsub P, Ittarat W, Kongkasuriyachai D, Uthaipibull C, et al. Role of Plasmodium vivax dihydropteroate synthase polymorphisms in sulfa drug resistance. Antimicrob Agents Chemother. $2016 \mathrm{Jul}$ 22;60(8):4453-63.

Poyraz O, Jeankumar VU, Saxena S, Schnell R, Haraldsson M, Yogeeswari P, et al. Structure-guided design of novel thiazolidine inhibitors of O-acetyl serine sulfhydrylase from Mycobacterium tuberculosis. J Med Chem. 2013 Aug 22;56(16):6457-66.

Quinn JG. (a) Evaluation of Taylor dispersion injections: determining kinetic/affinity interaction constants and diffusion coefficients in label-free biosensing. Anal Biochem. 2012 Feb 15;421(2):401-10.

Quinn JG. (b) Modeling Taylor dispersion injections: Determination of kinetic/affinity interaction constants and diffusion coefficients in label-free biosensing. Anal Biochem. 2012 Feb 15;421(2):391-400.

$\mathrm{R}$ Core Team. R: A language and environment for statistical computing. $\mathrm{R}$ foundation for statistical computing. 2013. Available from http://www.R-project.org/

Rabeh WM, Cook PF. Structure and mechanism of O-acetylserine sulfhydrylase. J Biol Chem. 2004 Jun 25;279(26):26803-6. 
Ramarao N, Nielsen-Leroux C, Lereclus D. The insect Galleria mellonella as a powerful infection model to investigate bacterial pathogenesis. J Vis Exp. 2012;(70):e4392.

Published 2012 Dec 11.

Reed P, Atilano ML, Alves R, Hoiczyk E, Sher X, Reichmann NT, et al. Staphylococcus aureus survives with a minimal peptidoglycan synthesis machine but sacrifices virulence and antibiotic resistance. PLoS Pathog. 2015 May 7;11(5):e1004891.

Reeve SM, Scocchera EW, N GD, Keshipeddy S., Krucinska J., Hajian B, et al. MRSA isolates from united states hospitals carry dfrg and dfrk resistance genes and succumb to propargyl-linked antifolates. Cell Chem. Biol. 2016;23, 1458-1467.

Rein MF, Elliot WC, Swenson JM, Thornsberry C. Sulfamethoxazole-trimethoprim synergism for neisseria gonorrhoeae, Antimicrobial agents and chemotherapy, 1980;17, 247-250.

Richter MF, Drown BS, Riley AP, Garcia A, Shirai T, Svec RL, et al. Predictive compound accumulation rules yield a broad-spectrum antibiotic. Nature. 2017 May 18;545(7654):299-304.

Romano KP, Ali A, Royer WE, Schiffer CA. Drug resistance against HCV NS3/4A inhibitors is defined by the balance of substrate recognition versus inhibitor binding. Proc Natl Acad Sci U S A. 2010 Dec 7;107(49):20986-91.

Saha K, Kapoor L, Arora VM, Chattopadhya D. Malaria, leprosy and dapsone. Southeast Asian J Trop Med Public Health. 2003 Sep;34(3):501-4.

Salsi E, Bayden AS, Spyrakis F, Amadasi A, Campanini B, Bettati S, et al. A. Design of O-acetylserine sulfhydrylase inhibitors by mimicking nature. J Med Chem. 2010 Jan 14;53(1):345-56.

Sander P, Springer B, Prammananan T, Sturmfels A, Kappler M, Pletschette M, et al. Fitness cost of chromosomal drug resistance-conferring mutations. Antimicrob Agents Chemother. 2002 May;46(5):1204-11.

Schnell R, Oehlmann W, Singh M, Schneider G. Structural insights into catalysis and inhibition of O-acetylserine sulfhydrylase from Mycobacterium tuberculosis. Crystal structures of the enzyme alpha-aminoacrylate intermediate and an enzyme-inhibitor complex. J Biol Chem. 2007 Aug 10;282(32):23473-81.

Schnell R, Sriram D, Schneider G. Pyridoxal-phosphate dependent mycobacterial cysteine synthases: Structure, mechanism and potential as drug targets. Biochim Biophys Acta. 2015 Sep;1854(9):1175-83. 
Scocchera E, Reeve SM, Keshipeddy S, Lombardo MN, Hajian B, Sochia AE, et al. Charged nonclassical antifolates with activity against Gram-positive and Gram-negative pathogens. ACS Med Chem Lett. 2016;7(7):692-696.

Sekowska A, Kung HF, Danchin A. Sulfur metabolism in Escherichia coli and related bacteria: Facts and fiction. J Mol Microbiol Biotechnol. 2000 Apr;2(2):145-77.

Shaw GX, Li Y, Shi G, Wu Y, Cherry S, Needle D, et al. Structural enzymology and inhibition of the bi-functional folate pathway enzyme HPPK-DHPS from the biowarfare agent Francisella tularensis. FEBS J. 2014 Sep;281(18):4123-37.

Silver LL. A Gestalt approach to Gram-negative entry. Bioorg Med Chem. 2016 Dec 15;24(24):6379-6389.

Silver LL. Challenges of antibacterial discovery. Clin Microbiol Rev. 2011;24(1):71109.

Singh S, Sablok G, Farmer R, Singh AK, Gautam B, Kumar S. Molecular dynamic simulation and inhibitor prediction of cysteine synthase structured model as a potential drug target for trichomoniasis. Biomed Res Int. 2013;2013:390920.

Sköld O. Sulfonamide resistance: Mechanisms and trends. Drug Resist Updat. 2000 Jun;3(3):155-160.

Spyrakis F, Singh R, Cozzini P, Campanini B, Salsi E, Felici P, et al. Isozyme-specific ligands for O-acetylserine sulfhydrylase, a novel antibiotic target. PLoS One. 2013 Oct 22;8(10):e77558.

Steiner EM, Böth D, Lössl P, Vilaplana F, Schnell R, Schneider G. CysK2 from Mycobacterium tuberculosis is an O-phospho-L-serine-dependent S-sulfocysteine synthase. J Bacteriol. 2014 Oct;196(19):3410-20.

Strebhardt K, Ullrich A. Paul Ehrlich's magic bullet concept: 100 years of progress. Nat Rev Cancer. 2008 Jun;8(6):473-80.

Tacconelli E, Carrara E, Savoldi A, Harbarth S, Mendelson M, Monnet DL, et al. WHO Pathogens Priority List Working Group. Discovery, research, and development of new antibiotics: the WHO priority list of antibiotic-resistant bacteria and tuberculosis. Lancet Infect Dis. 2018 Mar;18(3):318-327.

Tacconelli E, Sifakis F, Harbarth S, Schrijver R, van Mourik M, Voss A, et al. EPI-Net COMBACTE-MAGNET Group.. Surveillance for control of antimicrobial resistance. Lancet Infect Dis. 2018 Mar;18(3):e99-e106. 
Tamae C, Liu A, Kim K, Sitz D, Hong J, Becket E, et al. JH. Determination of antibiotic hypersensitivity among 4,000 single-gene-knockout mutants of Escherichia coli. J Bacteriol. 2008 Sep;190(17):5981-8.

Thiede JM, Kordus SL, Turman BJ, Buonomo JA, Aldrich CC, Minato Y, et al. Targeting intracellular $\mathrm{p}$-aminobenzoic acid production potentiates the anti-tubercular action of antifolates. Sci Rep. 2016;6:38083. Published 2016 Dec 1.

Toleman MA, Bennett PM, Bennett DM, Jones RN, Walsh TR. Global emergence of trimethoprim/sulfamethoxazole resistance in Stenotrophomonas maltophilia mediated by acquisition of sul genes. Emerg Infect Dis. 2007 Apr;13(4):559-65.

Trefouel J, Nitti F, Bovet D. Activité du p. aminophénylsulfamide sur l'infection streptococcique expérimentale de la souris et du lapin. C. R. Soc. Biol. 1935; 120:756.

Tsai CJ, Loh JM, Proft T. Galleria mellonella infection models for the study of bacterial diseases and for antimicrobial drug testing. Virulence. 2016 Apr 2;7(3):214-29.

Turnbull AL, Surette MG. L-Cysteine is required for induced antibiotic resistance in actively swarming Salmonella enterica serovar Typhimurium. Microbiology. 2008 Nov;154(Pt 11):3410-9.

Ventola CL. The antibiotic resistance crisis: part 1: Causes and threats. P T. 2015 Apr;40(4):277-83.

Waldron TT, Murphy KP. Stabilization of proteins by ligand binding: application to drug screening and determination of unfolding energetics. Biochemistry. 2003 May 6;42(17):5058-64.

Wellington S, Nag PP, Michalska K, Johnston SE, Jedrzejczak RP, Kaushik VK, et al. A small-molecule allosteric inhibitor of Mycobacterium tuberculosis tryptophan synthase.

Nat Chem Biol. 2017 Sep;13(9):943-950.

Westrop GD, Goodall G, Mottram JC, Coombs GH. Cysteine biosynthesis in Trichomonas vaginalis involves cysteine synthase utilizing O-phosphoserine. J Biol Chem. 2006 Sep 1;281(35):25062-75.

Williams KJ. The introduction of 'chemotherapy' using arsphenamine - the first magic bullet. J R Soc Med. 2009;102(8):343-348.

Wright GD. Q\&A: Antibiotic resistance: Where does it come from and what can we do about it? BMC Biol. 2010 Sep 20;8:123.

Yang J, Ogawa Y, Camakaris H, Shimada T, Ishihama A, Pittard AJ. folA, a new member of the TyrR regulon in Escherichia coli K-12. J Bacteriol. 2007

Aug;189(16):6080-4. 
Yao J, Ericson ME, Frank MW, Rock CO. Enoyl-acyl carrier protein reductase I (FabI) is essential for the intracellular growth of Listeria monocytogenes. Infect Immun. 2016 Nov 18;84(12):3597-3607.

Yun MK, Wu Y, Li Z, Zhao Y, Waddell MB, Ferreira AM, et al. Catalysis and sulfa drug resistance in dihydropteroate synthase. Science. 2012 Mar 2;335(6072):1110-4.

Zetola N, Francis JS, Nuermberger EL, Bishai WR. Community-acquired meticillinresistant Staphylococcus aureus: an emerging threat. Lancet Infect Dis. 2005 May;5(5):275-86.

Zhang JH, Chung TD, Oldenburg KR. A simple statistical parameter for use in evaluation and validation of high throughput screening assays. J Biomol Screen. 1999;4(2):67-73.

Zhao Y, Hammoudeh D, Yun MK, Qi J, White SW, Lee RE. Structure-based design of novel pyrimido[4,5-c]pyridazine derivatives as dihydropteroate synthase inhibitors with increased affinity. ChemMedChem. 2012 May;7(5):861-70.

Zhao Y, Shadrick WR, Wallace MJ, Wu Y, Griffith EC, Qi J, et al. Pterin-sulfa conjugates as dihydropteroate synthase inhibitors and antibacterial agents. Bioorg Med Chem Lett. 2016 Aug 15;26(16):3950-4.

Zheng J, Rubin EJ, Bifani P, Mathys V, Lim V, Au M, et al. Camacho LR. paraAminosalicylic acid is a prodrug targeting dihydrofolate reductase in Mycobacterium tuberculosis. J Biol Chem. 2013 Aug 9;288(32):23447-56.

Zhou Y, Joubran C, Miller-Vedam L, Isabella V, Nayar A, Tentarelli S, et al. Thinking outside the "bug": a unique assay to measure intracellular drug penetration in gramnegative bacteria. Anal Chem. 2015 Apr 7;87(7):3579-84.

Zlitni S, Ferruccio LF, Brown ED. Metabolic suppression identifies new antibacterial inhibitors under nutrient limitation. Nat Chem Biol. 2013 Dec;9(12):796-804 


\section{VITA}

Miranda J. Wallace was born in Pocahontas, Arkansas in 1988. Miranda received her Bachelor of Science in Chemistry at Arkansas State University in 2011. She received the Student Undergraduate Research Fellowship granted by the Arkansas Department of Higher Education to fund her undergraduate research under the supervision of Dr. Maureen Dolan. After graduating from Arkansas State University, Miranda continued her research career in the Dolan Lab at the Arkansas Biosciences Institute as a laboratory technician until 2013. In 2013, she was accepted into the Integrated Biomedical Sciences Ph.D. program at the University of Tennessee Health Science Center and began her graduate studies following the Microbiology, Immunology, and Biochemistry track. In the spring of 2014 she initiated her doctoral research in the lab of Dr. Richard E. Lee in the Chemical Biology and Therapeutics Department of St. Jude Children's Research Hospital. She produced four research articles during her time in the Lee Lab. During her graduate studies, Miranda served on the Graduate Student Executive Committee as both Professional Development Committee Chair and the Honor Council President. For her service and leadership at the University, Miranda was inducted into the Imhotep Society. Furthermore, for her research accomplishments and field of study, she was selected to join the Center for Pediatric Experimental Therapeutics scholars program. After the approval of her dissertation, Miranda will receive her doctorate from the University of Tennessee Health Science Center and graduate in August of 2019.

\section{List of Publications}

Garza I, Wallace MJ, Fernando D, Singh A, Lee RE, Gerding JS, et al. Synthesis and evaluation of thiazolidine amide and N-thiazolyl amide fluoroquinolone derivatives. Arch Pharm (Weinheim). 2017 Jun;350(6).

Griffith EC, Wallace MJ, Wu Y, Kumar G, Gajewski S, Jackson P, et al. The structural and functional basis for recurring sulfa drug resistance mutations in Staphylococcus aureus dihydropteroate synthase. Front Microbiol. 2018 Jul 17;9:1369.

Tsutsumi LS, Elmore JM, Dang UT, Wallace MJ, Marreddy R, Lee RB, et al. Solidphase synthesis and antibacterial activity of cyclohexapeptide wollamide B analogs. ACS Comb Sci. 2018 Mar 12;20(3):172-185.

Zhao Y, Shadrick WR, Wallace MJ, Wu Y, Griffith EC, Qi J, et al. Pterin-sulfa conjugates as dihydropteroate synthase inhibitors and antibacterial agents. Bioorg Med Chem Lett. 2016 Aug 15;26(16):3950-4. 